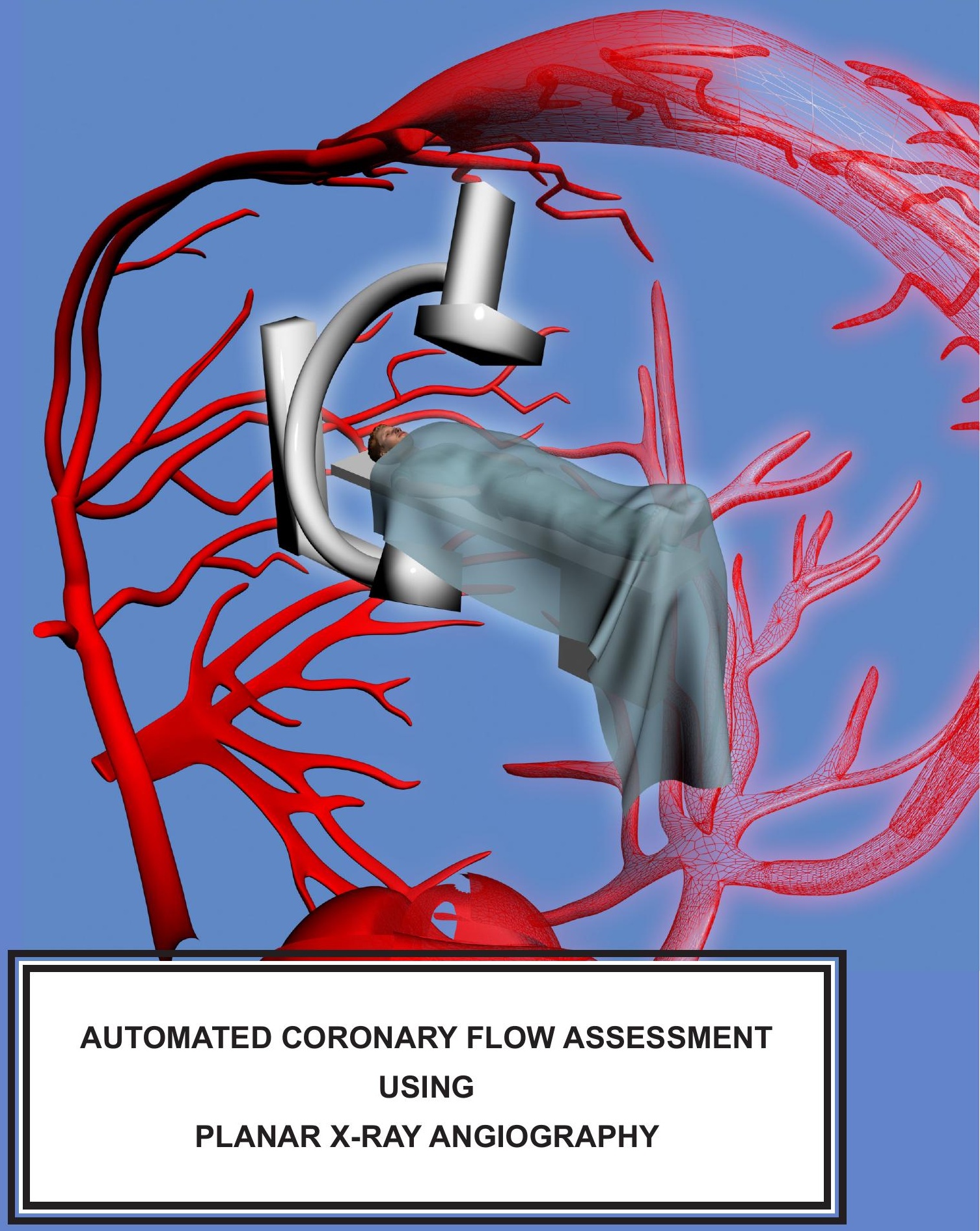




\section{AUTOMATED CORONARY FLOW RESERVE ASSESSMENT USING PLANAR X-RAY ANGIOGRAPHY}

Gerhard Albert ten Brinke 
De promotiecommissie:

voorzitter en secretaris:

Prof.dr.ir. A.J. Mouthaan Universiteit Twente promotor:

Prof.dr.ir. C.H. Slump

Universiteit Twente leden:

Prof.dr. C. von Birgelen

Universiteit Twente

Prof.dr.ir. G.J. Verkerke

Universiteit Twente

Prof.dr. A.G.J.M. van Leeuwen

Universiteit van Amsterdam

Dr. C.J. Storm

Dr. ir. A.M. Versluis

St. Franciscus Gasthuis, Rotterdam

Universiteit Twente

This research is financially supported by the Non-Invasive Molecular Tumor Imaging and Killing (NIMTIK) spearhead program of the University of Twente.

Signals \& Systems group,

EEMCS Faculty, University of Twente

P.O. Box 217, 7500 AE Enschede, the Netherlands

Print: Ipskamp Drukkers

Typesetting: LATEX2e

(c) Gerhard Albert ten Brinke, Enschede, 2011

No part of this publication may be reproduced by print, photocopy or any other means without the permission of the copyright owner.

ISBN 978-90-365-3244-0

DOI 10.3990/1.9789036532440 


\section{AUTOMATED CORONARY FLOW RESERVE ASSESSMENT USING PLANAR X-RAY ANGIOGRAPHY}

\section{PROEFSCHRIFT}

ter verkrijging van

de graad van doctor aan de Universiteit Twente, op gezag van de rector magnificus, prof. dr. H. Brinksma, volgens besluit van het College voor Promoties in het openbaar te verdedigen op 9 december 2011 om 12:45h

door

Gerhard Albert ten Brinke geboren op 21 September 1979

te Enter 
Dit proefschrift is goedgekeurd door de promotor:

Prof.dr.ir. C.H. Slump 


\section{Summary}

Cardiac and vascular diseases are the second cause of death in the Netherlands. Coronary lesions like stenosis, blocking the blood supply to the heart muscle, contribute significantly to the cause of heart failure. In this research we propose a method to quantify coronary stenosis using planar x-ray angiography image processing. The method we present here has a clinical equivalent called Thrombolysis in Myocardial Infarction (TIMI) frame counting. TIMI flow grade is a valuable and widely used qualitative measure in angiographic imaging. Since the flow grades are subjective and intra observer variability can be large, Gibson et al. introduced an associated quantitative method called the TIMI Frame Count (TFC). The TFC is measured by counting the number of frames between injection of the contrast agent in the artery until it reaches the end of the artery, which is recognized by the most distal landmark, often a bifurcation. The ratio of coronary flow velocities in basal and hyperemic conditions is a measure of coronary flow velocity reserve, which is a condition or quality measure of the heart.

In this thesis we aim at designing a robust algorithm for contrast flow tracking in multiple $\mathrm{x}$-ray image sequences. This thesis consist of five parts:

The first part concentrates on finding and tracking coronary arteries in a single 2-d x-ray image sequence. The contrast density is measured using regions of interests equispaced on the centerline of the arteries. From these measurements we create and analyze time density curves, which we use to estimate coronary flow velocity by locating the arrival and disappearance of the contrast agent using signal processing.

The second part focuses on the generation of a flow map, this is in fact a 2 -d representation of a set of time density curves, which is used to analyze contrast agent propagation using appropriate image processing techniques. With flow maps we are able to analyze the contrast density along the vessel centerline 
with a higher spatial resolution compared to using only a limited number of regions of interest.

The 3 -d reconstruction of the coronary arteries using primary and secondary image sequences obtained from two distinct projection angles is discussed in the third part. Here, we use computer vision techniques to create the coronary models. Although the input of the reconstruction algorithm consists of uncalibrated data, we manage to obtain 3-d models using semi-automatic annotation.

In the fourth part we use these 3 -d reconstructed coronary models to measure contrast density on hyperemic and basal image sequences. Important in this chapter is the ability to measure the length of the coronary arteries, which is eventually used to quantify the coronary flow velocity. The length measurements using the algorithm are compared to clinical catheter measurements. This results in an average error smaller than $20 \%$.

The final chapter concludes this research with a comparison of the algorithm to clinical results on a dedicated clinical dataset used for TIMI frame counting. From nine patients we had sufficient data to create a 3 -d model of the whole cardiac cycle and to use this model to measure coronary flow velocity. From these nine patients the algorithm correlates with $\mathrm{r}=0.98(\mathrm{P}<0.0001,95 \%$ CI 0.92 to 0.99$)$ to the clinical measurements of the coronary flow velocity using TIMI frame counting. 


\section{Samenvatting}

Hart en vaatziekten zijn de tweede doodsoorzaak in Nederland. Vernauwingen in kransslagaderen kunnen ontstaan door accumulatie van plak, om uiteindelijk het volledige bloedvat te blokkeren. Dit kan resulteren in een hartinfarct waardoor hartweefsel afsterft en hartfunctie afneemt, of het hart compleet doet stoppen met de dood tot gevolg.

In dit proefschrift beschrijven we de mogelijkheden om met behulp van beeldbewerkingstechnieken 2-d x-ray angiografie beeldreeksen te analyseren. De methode die wij hiervoor gebruiken heeft het klinische equivalent Thrombolysis in Myocardial Infarction (TIMI) stroming gradatie. De TIMI stroming gradatie is een waardevolle en veelgebruikte methode om een kwalitatieve indicatie te krijgen van de toestand van de kransslagaderen. Helaas is de TIMI stroming gradatie een subjectieve methode en kan de uitkomst nogal verschillen tussen artsen. Daarom hebben Gibson et al. de TIMI frame counting methode geïntroduceerd, waarbij het aantal beelden wordt geteld tussen het injecteren van de contrastvloeistof tot het arriveren ervan aan het einde van de kransslagader. De verhouding tussen het aantal frames in de basal en hyperemische toestand is een maat voor de reservecapaciteit van de kransslagader.

In dit onderzoek hebbben we een robust algoritme ontwikkeld om de contrastvloeistof in meerdere röntgenbeelden te volgen. Dit proefschrift is opgesplitst in vijf onderdelen. Het eerste deel concentreert zich op het detecteren en volgen van kransslagaderen in een enkele 2-d beeldreeks. Vervolgens wordt de contrastdichtheid gemeten met behulp van op gelijke afstand verdeelde meetregio's. Van deze meetregio's wordt een tijd-dichtheids grafiek gemaakt, waaruit wordt afgeleid hoe lang de contrastvloeistof erover doet om door de ader te stromen. Hieruit blijkt dat het lastig is om de contrast overgangen te vinden, dit kan verholpen worden door meerdere meetpunten mee te nemen. De focus van het tweede deel ligt op het genereren van een flowmap. Dit is een 
2-d representatie van contrastvloeistofmetingen op de middellijn van de aderen, uitgezet tegen de tijd. Door middel van beeldbewerkingstechnieken wordt hier de propagatiesnelheid van de contrastvloeistof uit afgeleid. De methode werkt beter dan de methode met een gelimiteerd aantal meetregios, omdat de maximale hoeveelheid meetpunten wordt gebruikt. In het derde onderdeel maken we de stap van 2-d naar 3-d door het construeren van een 3-d model van de kransslagaderen. We maken gebruik van computer vision technieken, die nodig zijn om de 2-d beelden om te zetten in 3-d informatie. Na goede resultaten op een fantoom is getest met klinische beelden, ook met deze data konden we, ondanks het missen van calibratie gegevens, 3-d modellen construeren. Hiervoor hebben we echter wel gebruik moeten maken van annotatie op kritische punten zoals katheter-eindpunt en vaat-bifurcaties. In het vierde deel wordt ingegaan op het maken van een 3 -d model met de informatie van de complete hartcyclus. Dit model kan wordt vervolgens gebruikt om lengtemetingen van de kransslagaderen te gebruiken voor het kwantificeren van de doorstromingsmetingen. Omdat er minimaal twee projectiehoeken noodzakelijk zijn voor een 3 -d reconstructie, konden we van slechts 9 patienten een volledige hartcyclus van de kransslagaderen construeren. Van de overige 25 patienten was er onvoldoende informatie aanwezig. In het vijfde onderdeel worden de 3 -d modellen toegepast op metingen van de constrastdichtheid. Hiervoor wordt het model passend gemaakt op de 2-d röntgenbeelden. Er worden flowmaps gemaakt in hypereamisch en basale condities waarin de doorstroomsnelheid wordt bepaald. Vervolgens worden de resultaten vergeleken met de waarden die gevonden zijn met behulp van de methode die gebruikt is in de klinische praktijk. Van de 9 bruikbare modellen konden we de doorstroming meten. Het resultaat van deze metingen correleert $\mathrm{r}=0.98(\mathrm{P}<0.0001,95 \%$ CI 0.92 to 0.99) met de handmatige metingen uit de kliniek. 
Summary

Samenvatting $\quad$ iii

1 Introduction 1

1.1 Cardiac diseases . . . . . . . . . . . . . . . 1

1.2 Coronary arteries . . . . . . . . . . . . . . . . . . . 2

1.3 Assessment modalities .................. . . . . . 4

1.4 Research questions . . . . . . . . . . . . . . . 5

2 Clinical coronary assessment methods $\quad 7$

2.1 Introduction . . . . . . . . . . . . . . . . . 7

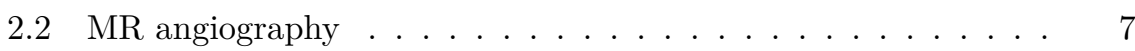

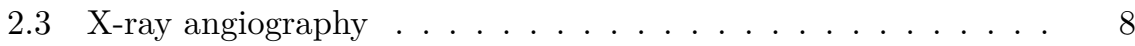

2.4 Contrast agent . . . . . . . . . . . . . . . . . 11

2.5 Flow models . . . . . . . . . . . . . . . . . 11

2.6 Analysis of x-ray angiograms . . . . . . . . . . . . . . . . . . 12

2.7 Functional diagnostics . . . . . . . . . . . . . . . . . . . . . . . . . . . 16

2.8 Treatments . . . . . . . . . . . . . . . 20

2.9 Quantitative coronary angiography ........... 22

3 Flow estimation using time density curves $\quad 27$

3.1 Introduction . . . . . . . . . . . . . . . . 27

3.2 Methods . . . . . . . . . . . . . . . . 29

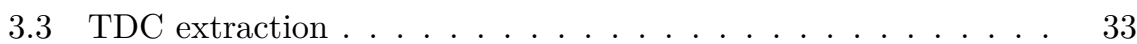

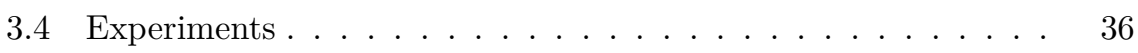

3.5 Results......................... 37 
3.6 Discussion and conclusion . . . . . . . . . . . . . . . 39

4 Flow estimation using a flow map 43

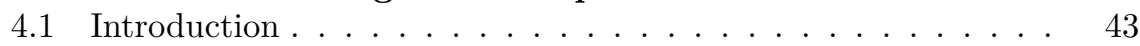

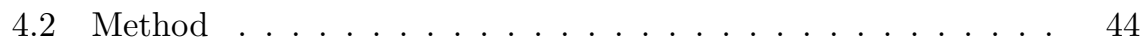

4.3 Experiments and results . . . . . . . . . . . . . . . . . . . 47

4.4 Discussion . . . . . . . . . . . . . . . . . . . . . . . . . . . . 47

4.5 Conclusion .......................... 49

5 Computer vision and coronary reconstruction $\mathbf{5 3}$

5.1 Introduction . . . . . . . . . . . . . . . . 53

5.2 Projective geometry . . . . . . . . . . . . . . 54

5.3 Camera matrix from C-arm geometry . . . . . . . . . . . 58

5.4 Essential and fundamental matrices . . . . . . . . . . . . . . 60

5.5 Three dimensional reconstruction methods . . . . . . . . . . 63

5.6 Experiments . . . . . . . . . . . . . . 67

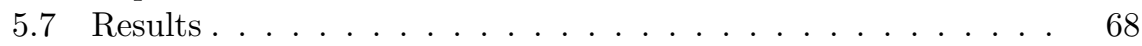

5.8 Discussion and conclusion . . . . . . . . . . . 68

6 Coronary modelling in 3-d $\quad \mathbf{7 3}$

6.1 Introduction . . . . . . . . . . . . . . . 73

6.2 Epipolar point matching . . . . . . . . . . . . . . 74

6.3 B-spline modeling . . . . . . . . . . . . . . . . . . . . . . . . . . . . . . . . 79

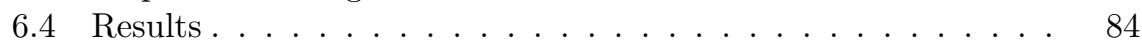

6.5 Conclusion ......................... 85

7 Flow estimation using 3-d reconstruction $\quad 87$

7.1 Introduction . . . . . . . . . . . . . . . . . . . 87

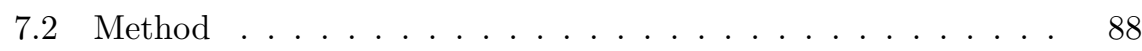

7.3 Experiments and results . . . . . . . . . . . . . . . . . . . 91

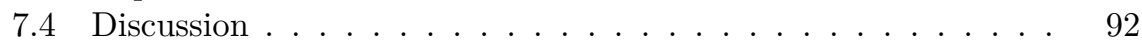

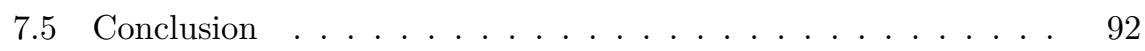

8 Automatic TIMI Frame counting using 3-d modeling 95

8.1 Introduction . . . . . . . . . . . . . . . . 95

8.2 Methods . . . . . . . . . . . . . . . 97

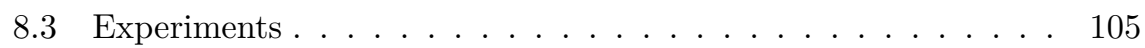

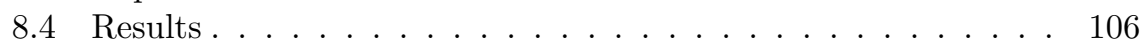

8.5 Discussion ........................... 109

9 Conclusion and Recommendations $\quad \mathbf{1 1 5}$

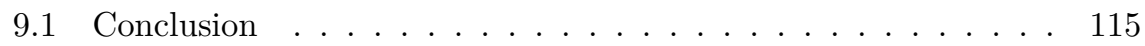

9.2 Recommendations ..................... 118

$\begin{array}{lr}\text { List of publications } & 119\end{array}$ 
A Datasets $\quad \mathbf{1 2 1}$

A.1 Dataset A . . . . . . . . . . . . . . . . . . . . . . . . . . . . . . 121

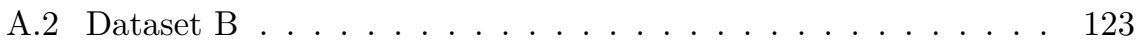




\section{Abbreviations}

CFVR Coronary flow Velocity Reserve, page. (20)

DICOM Digital Imaging and Communications in Medicine, page. (56)

DSA Digital Subtraction Angiography, page. (14)

ECG Electro-Cardiogram, page. (23)

FFR Fractional flow reserve, page. (16)

Ischaemia Receiving insufficient blood, page. (2)

IVUS Intravascular Ultrasound, page. (7)

LAD Left Anterior Descending, page. (2)

LCA Left Coronary Artery, page. (2)

LCx Left Circumflex, page. (2)

LM Left Main Artery, page. (14)

MRA Magnetic Resonance Angiography, page. (7)

Myocardium Heart Muscle, page. (2)

QCA Quantitative Coronary Angiography, page. (12)

RCA Right Coronary Artery, page. (2)

rCFVR relative Coronary flow Velocity Reserve, page. (20) 
TDC Time Density Curve, page. (23)

TFC TIMI Frame Count, page. (19)

TIMI Thrombolysis in Myocardial Infarction, page. (19)

TPR Total Peripheral Resistance, page. (16)

\section{Constants}

A Vessel cross-section, page. (11)

c Concentration of contrast agent after mixing, page. (11)

c $\quad$ Focal point, page. (56)

d $\quad 1 / 2$ size of detector [pixels], page. (56)

d Displacement vector, page. (60)

$\Delta p \quad$ Pressure drop, page. (16)

$\eta \quad$ Viscosity, page. (16)

E Essential matrix, page. (62)

F $\quad$ Fundamental matrix, page. (62)

$f \quad$ Focal distance, page. (56)

$f \quad$ Frequency in Hertz, page. (11)

- $\quad$ Normalized vector $\cdot$, page. (62)

I Injected amount of contrast agent, page. (11)

$J_{n} \quad$ Bessel functions of the first kind of order $n$, page. (11)

K Camera calibration matrix , page. (60)

L Tube length, page. (16)

$\mu \quad$ Pixel size $[\mathrm{mm}]$, page. (60)

$\nu \quad$ Kinematic viscosity, page. (11)

$\omega \quad$ Angular frequency in $\mathrm{rad} / \mathrm{sec}$, page. (11)

$p \quad$ Object point, page. (56)

$p^{\prime} \quad$ Projection of p, page. (56)

$\phi \quad$ Flow through a vessel or organ , page. (11) 
$h_{p f} \quad$ Impulse response of plug-flow, page. (11)

$h_{p} \quad$ Impulse response of Poiseuille-flow, page. (11)

$h_{v} \quad$ Impulse response of vessel-flow, page. (11)

$\mathbf{Q}(\mathbf{v}, \alpha)$ Quaternion rotation around $\mathrm{v}$ with angle $\alpha$, page. (57)

$Q \quad$ Volume flow, page. (16)

$R \quad$ Radius of cylinder or vessel, page. (11)

$R \quad$ Vascular resistance, page. (16)

$r \quad$ Tube radius, page. (16)

$\mathbf{R}_{\mathbf{x}}(\alpha)$ Euclidean rotation about $\mathrm{x}$ with angle $\alpha$, page. (57)

$\mathbf{R}_{\mathbf{y}}(\alpha)$ Euclidean rotation about y with angle $\alpha$, page. (57)

$\mathbf{R}_{\mathbf{z}}(\alpha)$ Euclidean rotation about $\mathrm{z}$ with angle $\alpha$, page. (57)

$s_{i} \quad$ Size of detector [mm], page. (56)

$\bar{t} \quad$ Mean transit time, page. (11)

$\bar{v} \quad$ Mean velocity, page. (11)

$V \quad$ Blood detectors, page. (11)

$v \quad$ Velocity in a vessel, page. (11)

$x \quad$ x-axis, page. $(56)$

$y \quad$ y-axis, page. (56)

$z \quad$ Z-axis, page. (56)

- $\quad$ Mean value $\cdot$, page. (57) 


\section{CHAPTER 1}

\section{Introduction}

The coronary arteries provide the heart with blood. This blood is vital to the operation of the heart, and when it is blocked or limited, it may result in heart failure. Coronary arteries can be made radio-opaque using contrast fluid, allowing the visualization using $x$-rays. In this thesis we propose a novel method towards a fully automated assessment of the functional behavior of coronary arteries by contrast fluid analysis.

\subsection{Cardiac diseases}

Cardiac and vascular diseases are, together with cancer, the main causes of death in the Netherlands. Figure 1.1 shows that cardiac and vascular diseases amount for $30 \%$ of the death cases in 2009. Accurate imaging is essential for assessing cardiac anatomy, function, perfusion and metabolism. The last 50 years has led to dramatic improvements in our ability to characterize and quantify disorders of the cardiovascular system [1].

Although our heart is pumping blood in large amounts through our body, it still requires separate arteries and veins for the supply of oxygen to the heart muscle. This myocardial perfusion is supplied by the coronary arteries, lying on the outer wall, or epicardial, of the heart. Coronary obstruction can occur when plague accumulates on the inside of the coronary vessel wall. Such an obstruction is called a stenosis. A clinical relevant stenosis reduces the myocardial blood flow, decreasing the supply of oxygen to the heart muscle. Subsequently, a stenosis can result in several types of heart problems:

- Angina pectoris: Pain in the chest which occurs when the heart receives 


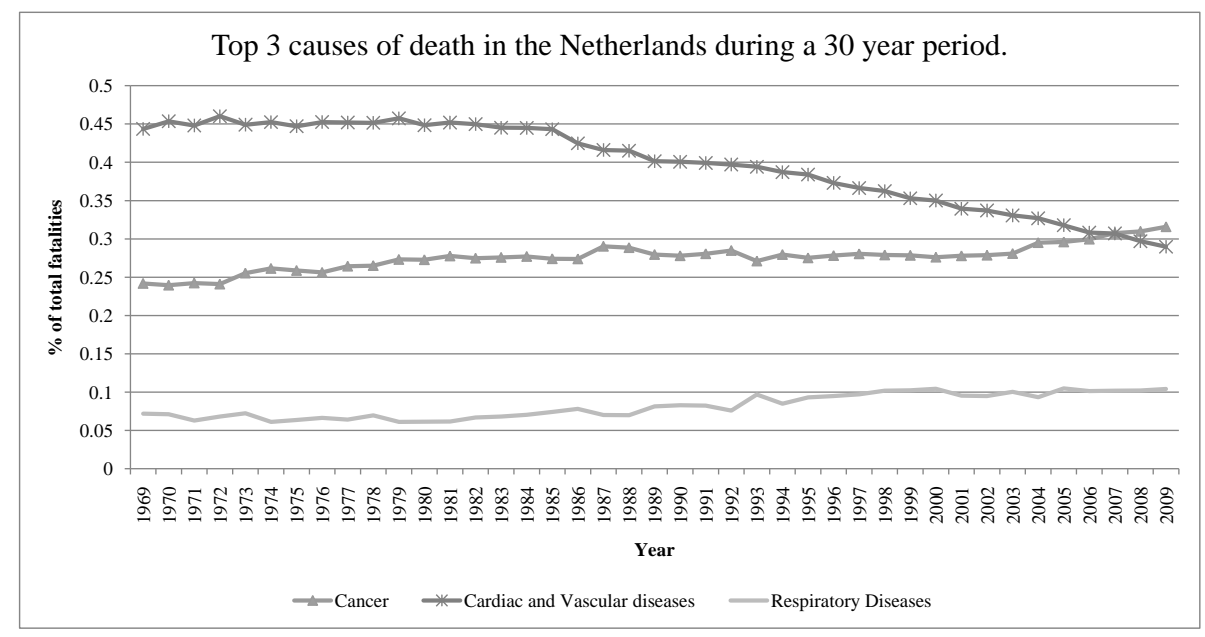

Figure 1.1: Causes of death in the Netherlands, source: Central bureau of statistics of the Netherlands

insufficient oxygen.

- Arrhythmias: Rhythm changes in the normal heart beat rhythm.

- Heart attack: When one of the coronary arteries is suddenly cut off, a heart attack follows. The blood flow to the heart muscle is stopped and an infarction follows. This causes permanent damage to the heart.

- Heart failure: In heart failure, the heart is not able to pump blood to the rest of the body effectively.

A heart muscle receiving insufficient blood is called an ischemic myocardium, and leads to myocardial oxygen shortage. A state of low oxygen is called hypoxia and no oxygen is called anoxia. Anoxia can cause permanent damage to the heart muscle, the so-called infarction. The next section describes the coronary arteries in more detail.

\subsection{Coronary arteries}

\subsubsection{Introduction}

Cardiac perfusion is depending on healthy coronary arteries. Figure 1.2 shows the coronary arteries of a human heart. The coronary arteries are branching from the aorta into the left coronary artery (LCA) and right coronary artery (RCA). The LCA starts, depending on physiological properties of the person, as the left main (LM) artery which is about $2 \mathrm{~cm}$ in length before it branches in the left circumflex (LCx) and left anterior descending (LAD) [2]. These 


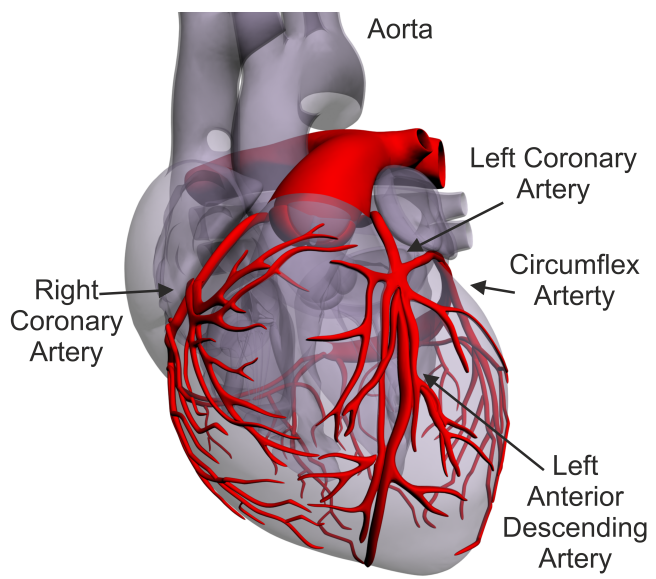

Figure 1.2: Coronary arteries, with annotation of the main branches.

epicardial located arteries are mainly filled with blood in the diastole stage (relaxation of the heart muscle), and they are compressed in the systole (heart contraction) stage. The RCA and LCA supply blood mainly to respectively the right and left ventricle of the heart.

\subsubsection{Blood supply regulation}

Vessels have a resistance depending on the radius of the vessel according to Poiseuille's Equation [3]. Assuming a constant pressure, this resistance limits the blood flow through a vessel. This effect can be visualized by looking at the aorta, which has a diameter of approximately $1.5 \mathrm{~cm} \mathrm{[2].} \mathrm{Coronary} \mathrm{arteries}$ have a size of approximately $0.5 \mathrm{~cm}$ and they transport less blood compared to the aorta. Blood flow is regulated by the arterioles. The arterioles are small, with a mean diameter of approximately $0.5 \mathrm{~mm}$, and control the blood flow by vasodilation or vasoconstriction. Vasoconstriction is the process when the smooth muscle contracts and decreases the diameter of the arteriole. Vasodilation has the inverse effect, the smooth muscle relaxes causing the arteriole to dilate. In normal body functioning this vasodilation is controlled by adenosine. After intra coronary administration of adenosine, the arterioles are dilated and more blood is transported to the myocardium. This mechanism is naturally initiated when the heart requires more oxygen.

Adenosine is also used in invasive diagnostics to induce dilation of the arterioles pharmacologically, but in our experiments papaverine is used as the dilating drug because of the longer dilation time of the arterioles compared to adenosine [1]. It may occur that an obstruction in an artery is naturally bypassed by means of small arteries starting before the obstruction and leading 


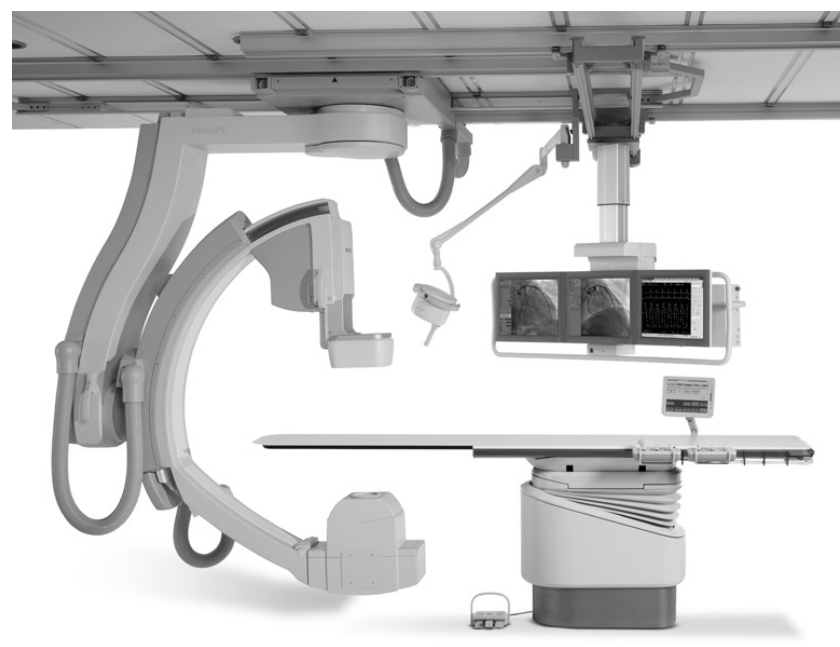

Figure 1.3: Philips Allura Xper FD 10, used to make X-ray angiography image sequences. [http://newscenter.philips.com]

after the obstruction. The clinical name for this bypass is collateral blood flow. The small size of the collaterals, 20-350 micrometers, make them invisible on the angiograms. Therefore it may not be necessary to remove the obstruction because the collaterals have taken over the function of the artery $[4,5]$.

\subsection{Assessment modalities}

A widely used modality for visualizing the coronary arteries is x-ray angiography. Compared to other modalities, for example, MR angiography, x-ray angiography equipment is smaller in size, less costly and delivers higher resolution images. Figure 1.3 shows the equipment used for patient assessment and intervention. While the patient is lying on the table, the cardiologist moves the C-arm, containing a x-ray source and on the other side a detector, around the patient to obtain a pronounced projection of the coronary arteries. The C-arm has two main degrees of freedom: the primary and secondary angle. The primary angle $\alpha$ ranges from right anterior oblique (RAO) $(\alpha>0)$ to left anterior oblique $(\mathrm{LAO})(\alpha<0)$ and the secondary angle $\beta$ range from cranial $(\beta>0)$ to caudal $(\beta<0)$. The cardiologist can put the table in floating mode. In this mode, the table can be moved in $x$ and $y$ direction freely. The table height, in $z$ direction, can be controlled by a motor.

Coronary arteries are made visible by opacification of the vascular structures using a contrast agent. A contrast bolus of $3-8 \mathrm{ml}$ is injected directly into the 
coronary arteries using a catheter which is typically inserted in the ascending aorta near the groin, or the femoral artery. The radio-opaque contrast agent shortly replaces the blood and absorbs the x-rays. The coronary arteries will show up on the angiograms shortly after injection with a duration of about 3-5 seconds. The physics of the equipment used to transform the x-ray projections to 2-d images is described in detail in section 2.3.3. Figure 2.6 shows a typical xray angiogram with a conspicuous projection of the left branch of the coronary arteries. The arrow points to the location of a narrowing of the artery, which can be identified as a stenosis.

\section{$1.4 \quad$ Research questions}

Analysis of blood flow velocity using quantitative coronary angiography can assist in a prediction of coronary stenosis severity. This thesis is structured using a bottom up approach to reach a solution for the estimation of coronary flow velocity. Many challenges in the image processing domain are present. Firstly, vessel detection in coronary angiograms is still a topic of research, since it is difficult to separate background structures and noise from the vessels. It is important to locate the vessels correctly, because they contain the information about the blood flow. Secondly, we need temporal information about the blood flow. This makes it necessary to find the vessels not only in one single image, but throughout a sequence of images. Furthermore, we have to relate the detected vessels in subsequent images. Temporal tracking is the main issue in which has to be solved. The motion present in the images originates not only from cardiac and breathing motion, but it can also involve table motion used for bolus chase by the cardiologist.

\section{Clinical coronary assessment methods}

In chapter 2 we continue with an introduction into x-ray angiography. The main research questions here are:

- How can we quantify coronary stenosis with substantial clinical relevance?

- What is the clinical equivalent of measuring coronary blood flow velocity from x-ray images?

\section{Finding time density curves}

The first step in the analysis of x-ray images is to find the coronary arteries. In chapter 3 we discuss how to use appropriate image processing algorithms to find and track vascular structures. The research questions here are:

- How can we automatically find the vascular structure in 2-d x-ray images? 
- How can we accurately extract time density curves?

- Are time density curves sufficient to determine coronary flow?

\section{Flow map introduction}

In chapter 4 we introduce the concept of a flow map, this is a 2 -d representation of a set of time density curves. We explore the usage of this flow map and answer the following research questions:

- How do we represent a set of time density curves?

- Can we estimate flow velocity from a flow map?

\section{3-d reconstruction of the coronary arteries}

Until now, we have only focused on 2-d image analysis. This is rather limited, since we are analyzing 3-d structures. Therefore we continue with the 3 - $\mathrm{d}$ reconstruction of the vascular information. In chapter 5 the mathematics of biplane reconstruction and the application to coronary x-ray images is discussed. The main research question answered in this chapter is:

- Contain x-ray images enough information to reconstruct 3-d models?

\section{4-d modeling of coronary arteries}

The next step is the creation of a time varying model of the coronary arteries and measure the contrast density using this 4-d model. The biggest challenge is the three dimensional reconstruction of the coronary vessel tree and subsequently the temporal modeling of the coronary vessel tree. Assuming that the temporal reconstruction results in a valid coronary model covering the whole cardiac cycle, a valid flow map can be created. This is done by fitting this model to the two dimensional image sequence with contrast enhanced coronary arteries. Measurement of the vessel centerline results in the flow map, which is used to measure coronary flow velocity.

- How can we create flow maps using a 4-d model of the coronary arteries during the whole cardiac sequence?

\section{Validation of our method using clinical data}

The final chapter of this thesis compares clinical and computer analyzed flow velocity estimations, in this chapter the following questions will be answererd:

- How accurate is the developed algorithm?

- What are the limitations the developed algorithm?

This thesis will continue in the next chapter with an introduction to clinical coronary assessments methods. 


\section{Clinical coronary assessment methods}

\subsection{Introduction}

Several modalities to detect arterial problems with patients are available. Magnetic Resonance Imaging (MRI) scans can be made to detect problems on arteries, also x-ray beams are used to image the arteries. There are more systems like intra-vascular ultrasound (IVUS) [6], but only MRI and x-ray is discussed briefly in this section.

\subsection{MR angiography}

During the last few years MRI (Magnetic Resonance Imaging) has improved considerably [7], still, for invasive diagnostics the method of choice is x-ray angiography. MRI applied for vascular imaging is called MRA (Magnetic Resonance Angiography). Intervention is limited while using MRA because the patient is almost entirely inside the scanning equipment. This is not the case in x-ray equipment. Also the price of MRA equipment is much higher than that of x-ray angiography equipment. This is caused by the magnetic coil required in MRA. MRA is a good method for diagnostics but has limitations during surgical intervention.

Tagged MRI [8] is a technique which allows motion field generation by following magnetic 'tags'. Myocardial tissue can be tagged with radio-frequency (RF) saturation before acquiring images. These tags can be used to label and track specific region during the cardiac cycle, resulting in a motion field which 
can be described by deformable models [9].

\subsection{X-ray angiography}

A widely used system for visualizing the coronary arteries is x-ray angiography. Injecting a contrast agent into the coronary arteries makes the arteries opaque. This contrast agent mixes with the blood and absorbs the x-rays. The $\mathrm{x}-$ ray beam, which is attenuated while it propagates through the body at the location of the heart, is projected on the intensifier or detector array. The intensifier amplifies the projection and transforms it to the visible domain. The attenuation of the $\mathrm{x}$-rays by the contrast agent results in an image where the intensity of the x-ray projection is lower. This attenuation results in a view of the coronary arteries. To determine the conditions of the coronary arteries, quantitative analysis of the x-ray images (angiograms) is required. Quantitative angiography analysis is performed on an image sequence obtained using x-ray scans of the heart. X-ray angiography is used most of the time during intervention. X-ray angiography will be described in detail in section 2.3.3.

\subsubsection{Rotational X-ray}

In rotational angiography, the $\mathrm{C}$-arm rotates a predefined path around the patient. During this rotation, or sweep, a number of frames are acquired. This acquisition may be synchronized to the ECG using prospective ECG gating. Prospective ECG gating will allow easier 3-D modeling since the images are acquired at the approximately the same phase of the cardiac cycle. Retrospective gating can be used to assign frames to a cardiac phase after the acquisition. This, however, may result in slightly off-phase acquisitions.

\subsubsection{CT Angiography}

Computed Tomography Angiography (CTA) is a three dimensional imaging modality. Images are acquired slice by slice using x-ray. The gantry rotates around the subject and measures the attenuation of the x-ray beams. The measured data is used to reconstruct a three-dimensional view of the subject. This method is most often based on filtered back projection. Detailed images of the arteries and veins can be produced using a contrast agent injected intravenously.

\subsubsection{X-ray angiography}

In this section we explain the physical and functional properties of x-ray equipment. A conventional X-ray imaging system as shown in figure 2.1a consists 
of an x-ray tube, a filter, a collimator, a grid, and an image intensifier or intensifying screen or film detector.

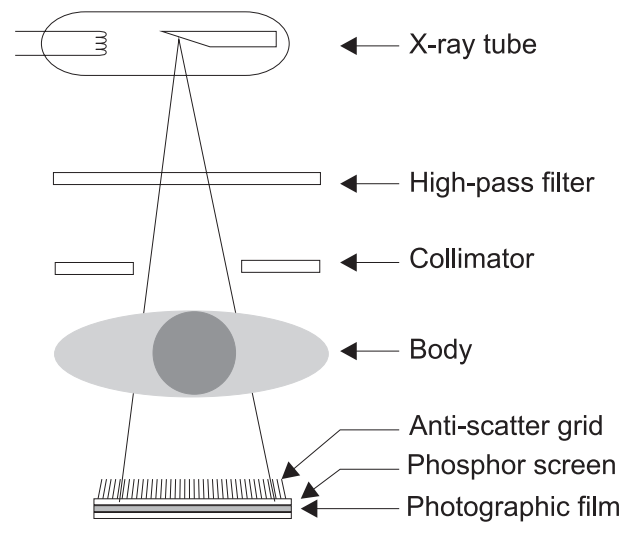

a: Conventional x-ray equipment

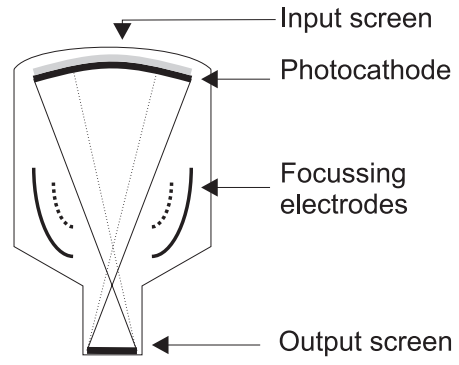

b: Image intensifier

Figure 2.1: X-ray system and image intensifier

The image intensifier converts x-ray into visible light. In figure $2.1 \mathrm{~b}$ a typical image intensifier is shown. The intensifier is a vacuum tube with walls made of glass or metal. X-ray photons enter at the input and fall on the input screen, where photons are created. The high-energy photons are converted to photons by the photo cathode. While the photons are accelerated to between $25 \mathrm{keV}$ and $40 \mathrm{keV}$ they are also focused by the electrostatic lens. The high energy photons produce thousands of photons at the output screen for each electron [10]. This visible light is then captured by a camera. In figure $2.1 \mathrm{~b}$ a dual mode image intensifier is drawn. Two sets of electrodes focus a larger or smaller portion of the input image onto the output screen, changing the Field of View (FOV). The collimator is an important part of the x-ray system when changing the FOV. It reduces the x-ray radiation not used for imaging purposes.

Two classes of detectors exist: direct en indirect conversion, see figure 2.2. Direct-conversion detectors convert $\mathrm{x}$-ray photons directly into an electric charge using an x-ray photo conductor, such as amorphous selenium. Indirectconversion detectors require a scintillator that first converts X-rays into visible light. The light can be converted into an electric charge using photo detectors like a CCD or amorphous silicon. The amorphous silicon layer can be readout using a Thin-Film transistor (TFT) array layer, which can also be used to readout the amorphous selenium layer used in direct-conversion detectors.

Modern systems, like the Siemens Axiom Artis dFC, use an indirect conversion detector which uses a cesium iodide (CsI) scintillator and a photo diode/TFT readout array. CsI is used to create a structured scintillator. A structured scintillator channels the visible light in contrast to unstructured scin- 


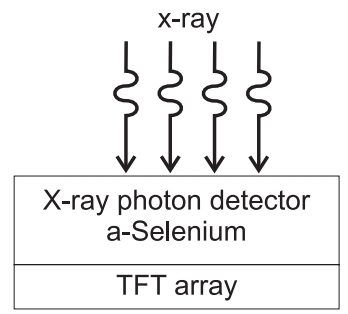

a: Direct

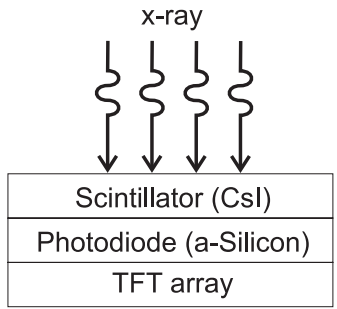

b: Indirect with CsI layer

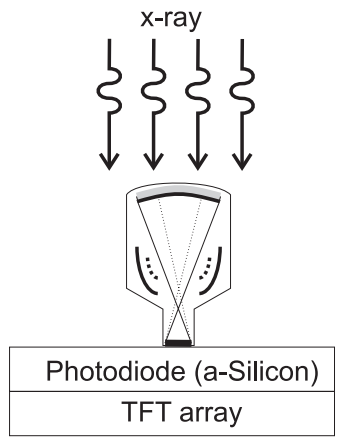

c: Indirect with image intensifier

Figure 2.2: Detector classes

tillators where the visible light is scattered in the material. A good overview of imaging systems using a flat panel semiconductor without a tube can be found in Rocha [11].

Although modern flat panel detectors do not suffer from geometrical distortion, there are some hazards: Modern detectors are completely controlled by an exposure computer: parameters like peak kilo voltage $(\mathrm{kVp})$, filament current $(\mathrm{mA})$ and pulse width are automatically set. The working range of flat detector is therefore extended compared to image intensifier systems. Nowadays, even in obese people, clear images can be produced. This, however, requires more intense x-ray exposure. For example: an increase in body diameter of $1 \mathrm{~cm}$ will result in a $25 \%$ dose increase. For $3 \mathrm{~cm}$ and $8 \mathrm{~cm}$ the dose increases even more with $50 \%$ and $596 \%$ respectively [12]. This can result unintendedly in a large $\mathrm{x}$-ray dose for the patient causing skin burns.

\subsubsection{Table panning}

In the past, cardiac angiographers used high magnifications and table panning to keep the vessels centered in the field of view. This was done because image intensifiers had the highest spatial resolution in the center of the image. At high magnification, a small portion of the curved intensifier was used reducing the amount of pincushion distortion [12]. In this thesis we assume that all images are acquired using a flat panel detector because image intensifiers are replaced by flat panel detectors in new equipment. From this assumption we can also create a table panning algorithm which is discussed in chapter 6 . 


\subsection{Contrast agent}

The contrast agent used in typical x-ray coronary angiography consists of a solution with $\approx 350 \mathrm{mg} / \mathrm{ml}$ iodine that must be injected into the coronary arteries. This is achieved using an needle with a syringe filled with $20 \mathrm{ml}$ contrast fluid [13]. For static imaging, the right coronary artery requires 5-8 $\mathrm{ml}$ of contrast fluid and the left coronary artery requires 8-10 ml. For rotational angiography, the amounts of contrast agent are slightly higher: $10-16 \mathrm{ml}$ and 6-10 $\mathrm{ml} \mathrm{LCA}$ and RCA respectively. In some patients, the contrast agent may induce some unwanted reactions:

- Urticaria or skin rash occurring mostly from allergic reactions.

- Angioedema, which is comparable with urticaria, but involves allergic reactions below the skin (swellings).

- Bronchospasm, which causes breathing problems.

- Hypotension or shock

Another group of reactions are the chemotoxic effects:

- Flushing

- Nausea

- Nephrotoxicity

- Pain during injection

- Vomiting

Therefore, it is important that we limit the amount of contrast agent, which imposes constraints on the image acquisition.

\subsection{Flow models}

Blood flow through a vessel can be modeled as fluid flow through a tube. Important parameters in the modeling of this process are summarized in table 2.1. There are several models describing the flow in a tube [2]. Womersley et al. [15] derived the axial component of the velocity $v(r, t)$ of biological fluid.

Finite element analysis including the Womersley equation for the velocity profile can gives an accurate solution to the velocity flow problem [16], but the practical use is limited. In practice, the contrast agent flow through a vessel on the vessel centerline can be expressed using simplified models. The impulse response of fluid flow through a tubular structure of length $L$ with $T_{L}$ the transit time $T_{L}=\frac{L}{v_{0}}$ can be simplified as plugflow $h_{p f}(t)$ :

$$
h_{p f}(t)=\delta\left(t-T_{L}\right)
$$

This is the basic model, extending this using Poiseuille's law results in the impulse response of Poiseuille flow $h_{p}(t)(2.2)$. The formula presented here does 


\begin{tabular}{|l|c|c|}
\hline Flow through a vessel or organ & $\phi$ & {$[\mathrm{ml} / \mathrm{min}]$} \\
Flow based on indicator dilution & $\phi=\frac{I}{\int c d t}$ & \\
$\quad I$ injected amount & $\phi=\bar{v} \cdot A$ & \\
$\quad c$ concentration after mixing & $\bar{l}=V / \bar{t}$ & $\bar{t}=\frac{\int c \cdot t d t}{\int c d t}$ \\
Flow based on mean velocity $\bar{v}$ and cross-section $A$ & $\frac{\phi}{\text { mass }}$ & {$[\mathrm{ml} / \mathrm{min} \cdot 100 \mathrm{~g}]$} \\
Flow based on blood volume between detectors $V$ and \\
mean transit time $\bar{t}$ & $\frac{\phi_{\text {hyperemic }}}{\phi_{\text {basal }}}$ & \\
Global or regional relative flow & $\frac{\phi \text { endo }}{\phi_{\text {epi }}}$ & \\
Flow reserve & $v(t)$ & \\
Transmural flow & $\frac{v_{\text {hyperemic }}}{v_{\text {basal }}}$ & \\
Velocity in a vessel & \\
Velocity reserve & \\
\hline
\end{tabular}

Table 2.1: Terminology, calculation and units for blood flow measurement, Table reproduced from $[14]$

not take the radius of the tube into account, but it shows the change in flow compared to the plug flow. The derivation of this formula can be found in [17].

$$
h_{p}(t)= \begin{cases}\frac{T_{L}}{t^{2}} & \text { for } t \geq T_{L}=L / v_{0} \\ 0 & \text { otherwise }\end{cases}
$$

A better representation of blood flow through coronary vessels is given by $\mathrm{M}$. Schrijver [17] by adjusting the Poiseuille flow model to realistic measurements of a contrast fluid propagating through a tube. The so-called vessel flow $h_{v}$ (2.3) is defined by:

$$
h_{v}(t)= \begin{cases}\frac{3}{T_{L}} \exp -3\left(\frac{t}{T_{L}}\right) & \text { for } t \geq T_{L} \\ 0 & \text { otherwise }\end{cases}
$$

Figure 2.3 shows the responses on an impulse and on the input $u(t)$ defined as $u(t)=1$ for $100<t<600 \mathrm{~ms}$ and $u(t)=0$ otherwise. It clearly shows the contrast density at the vessel centerline as a function of time and position.

\subsection{Analysis of x-ray angiograms}

\subsubsection{Quantitative coronary angiography (QCA)}

Densitometry is the common name of measuring contrast agent density in images. In theory, according to Beer-Lambert law (eq 2.4), the x-ray beam atten- 

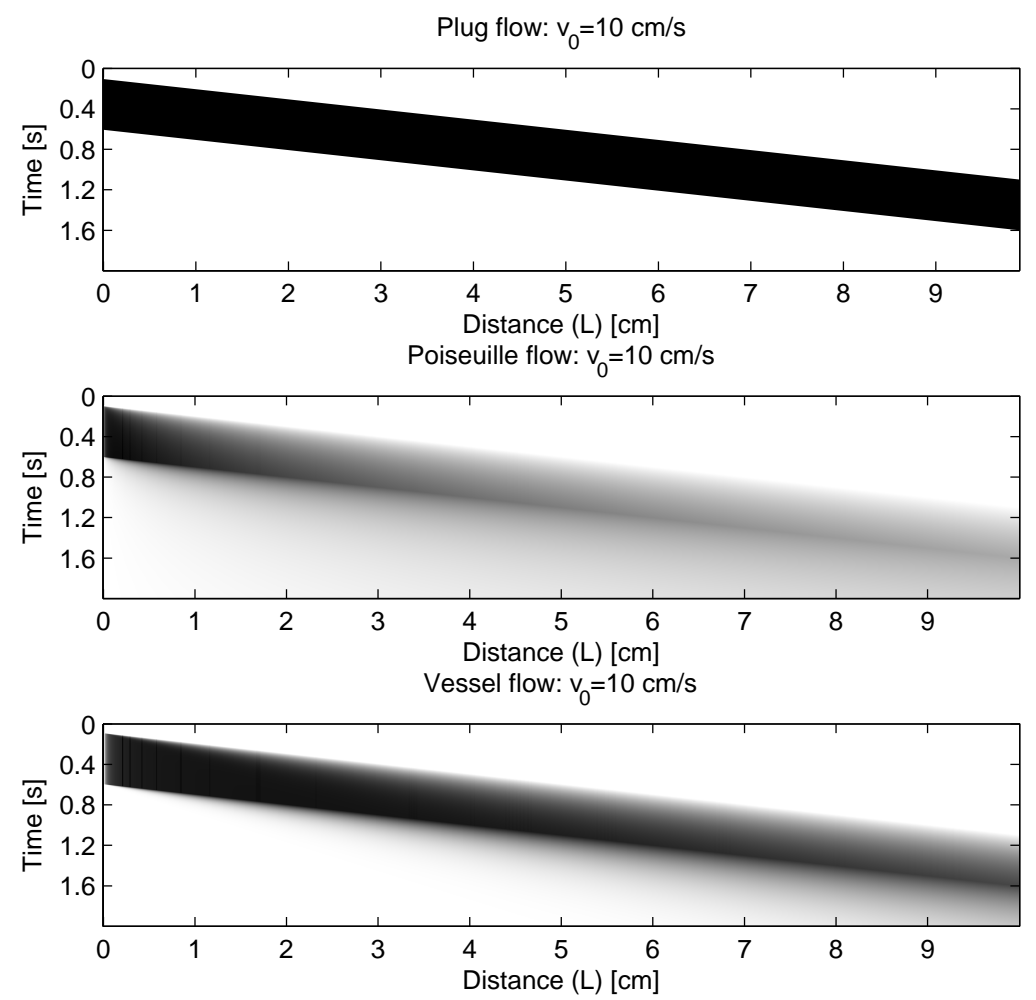

Figure 2.3: Plug $h_{p f}$, Poiseuille $h_{p}$ and vessel flow $h_{v}$ responses on input $u(t)$, which is defined as $u(t)=1$ for $100<t<600 \mathrm{~ms}$ and $u(t)=0$ otherwise.

uates through a vessel proportional to the thickness of the contrast inside the vessel.

$$
I=I_{0} e^{-\mu l}
$$

where $I_{0}$ is the intensity of the x-ray source, $I$ is the intensity of the x-ray at distance $l$ into the material and $\mu$ is the attenuation coefficient. Application in clinical practice is limited due to several error sources:

- Polyenergetic x-ray beam.

- Image intensifier characteristics:

- Veiling glare

- Vignetting

- Foreshortening and overlap of structures 
- Non-uniform radiographic magnification

- Non-uniform radiographic exposure (heel effect)

- Contrast distribution is equal in both normal and stenotic segments

In this thesis, we will not focus on the physical properties of the x-ray setup. Only the problems related to coronary modeling, which is the foreshortening and overlap of structures, will be discussed.

\subsubsection{Digital Subtraction Angiography}

Digital Subtraction Angiography (DSA) is a method to enhance the visibility of arteries. This is done by subtracting a pre contrast image from a post contrast image using equation 2.5 with $I_{D S A}$ is the resulting image, $I$ is the image on which DSA is applied and $I_{\text {Precontrast }}$ is the image obtained in the phase where no contrast fluid is absorbing x-ray signals.

$$
I_{D S A}=G\left(\log (I)-\log \left(I_{\text {precontrast }}\right)\right)+r
$$

where $G$ is a gain factor and $r$ is the offset. In practice of coronary angiography, DSA is quite challenging because of the continuous motion of the heart, body (respiration) and of the table. Table motion occurs when the cardiologist positions the table to put the heart of the patient in view of the detector. Body respiration is in practice not a problem because most of the patients can hold their breath for 40 seconds, however for old people, which are the largest group of patients, this can be a problem. Shechter et al. [18] goes into detail on the respiratory motion on coronary angiograms. Also the motion of the heart can be compensated for by using a post contrast image sequence of a cardiac cycle as a DSA image sequence, but this only works with a constant cardiac cycle and ECG gating. Background artifacts can be suppressed by using the average of all images $\bar{I}$ as mask. This method creates images with less background noise, but also introduces motion blur artifacts.

\subsubsection{Optimal views}

Vessel tortuosity and curvature is an important measure used in stent placement. Stent delivery systems must be able to reach the location of the lesion safely. This information can be obtained from x-ray images using an optimal view [20]. Optimal views of the coronary arteries are obtained using some predefined projection angles. Table 2.2 shows typical projections. Examples of these projections are shown in figure 2.4 for the left coronary artery (LCA) and figure 2.5 for the right coronary artery (RCA). All images are acquired from the same patient. Optimal views prediction is an important application of coronary models created using 3DRA or CTA equipment. 


\begin{tabular}{|c|c|c|c|c|}
\hline \multicolumn{5}{|c|}{ Left coronary artery } \\
\hline $20^{\circ}$ & $\mathrm{RAO}$ & $20^{\circ}$ & CAUD & LM and LCx \\
\hline $0^{\circ}$ & PA & $40^{\circ}$ & CRAN & $\mathrm{LAD}$ \\
\hline $45^{\circ}$ & $\mathrm{LAO}$ & $30^{\circ}$ & CRAN & LAD and diagonals \\
\hline $30^{\circ}$ & $\mathrm{RAO}$ & $30^{\circ}$ & CRAN & $\mathrm{LAD}$ \\
\hline $45^{\circ}$ & $\mathrm{LAO}$ & $30^{\circ}$ & CAUD & $\begin{array}{l}\mathrm{LM} \text {, proximal } \mathrm{LAD} \text {, proximal } \mathrm{LCx} \text {, and collaterals } \\
\text { to the } \mathrm{RCA}\end{array}$ \\
\hline \multicolumn{5}{|c|}{ Right coronary artery } \\
\hline $40^{\circ}$ & $\mathrm{LAO}$ & $0^{\circ}$ & \multirow{3}{*}{ CRAN } & Proximal and mid-RCA \\
\hline $0^{\circ}$ & PA & $40^{\circ}$ & & Distal RCA (PDA and PV branches) \\
\hline $35^{\circ}$ & $\mathrm{RAO}$ & $0^{\circ}$ & & Proximal and mid-RCA \\
\hline
\end{tabular}

Table 2.2: Table with common projection angles (in degrees). With LAD = left anterior descending; LAO = left anterior oblique; LCx = left circumflex; LM = left main; $\mathrm{PA}=$ posteroanterior; $\mathrm{PDA}=$ posterior descending arterty; $\mathrm{PV}=$ pulmonary vascular; $\mathrm{RAO}=$ right anterior oblique; $\mathrm{RCA}=$ right coronary artery; $\mathrm{CAUD}=$ caudal; CRAN = cranial. Table reproduced from Aviles et al.[19]

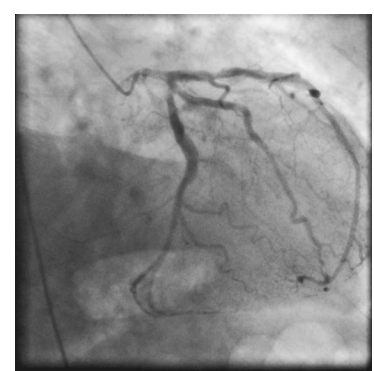

a: $33^{\circ} \mathrm{RAO}, 27^{\circ} \mathrm{CAUD}$

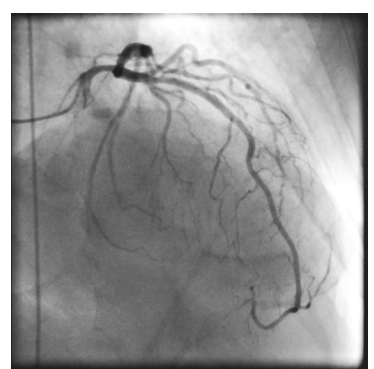

b: $25^{\circ} \mathrm{RAO}, 25^{\circ} \mathrm{CRAN}$

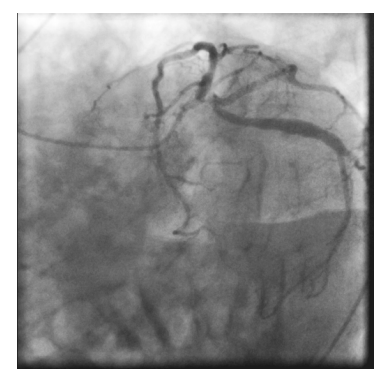

c: $40^{\circ} \mathrm{LAO}, 34^{\circ} \mathrm{CAUD}$

Figure 2.4: Examples of common projections of the left coronary artery. All images are from the same patient.

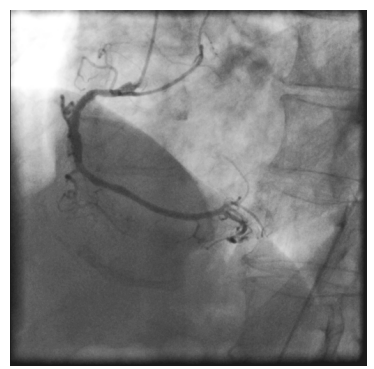

a: $48^{\circ} \mathrm{LAO}, 0^{\circ} \mathrm{CAUD}$

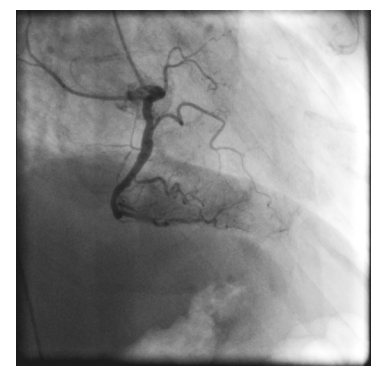

b: $49^{\circ} \mathrm{RAO}, 0^{\circ} \mathrm{CAUD}$

Figure 2.5: Examples of common projections of the right coronary artery. All images are from the same patient. 


\subsubsection{Motion}

Motion originates from several sources. First of all there is cardiac motion, which has a periodic cycle that can be measured using the ECG. Second motion is patient respiration. This motion can be approximately corrected by a translation in the image plane. The third motion is due to table movement. The physician has the ability to float the table during image acquisition, which also results in translations in the image plane.

\subsubsection{Applications of QCA}

Currently, QCA can be applied in clinical practice to reliably assess coronary lumen diameter and length. Assessment of vessel topology like bifurcations also aids intervention procedures like stent placement.

The generation of a 3-D coronary model can provide important anatomical characteristics like vessel curvature, torsion, and bifurcations angles that may not be assessed reliable using 2-D angiography.

\subsection{Functional diagnostics}

\subsubsection{Vasodilation}

The health-state of the heart can be measured by inducing the condition that the heart is working at its maximum capacity. This condition is characterized by a maximum blood uptake and is called the hyperemic condition. To mechanically allow maximum blood uptake, vessels must be widened or dilated. This vasodilation directly affects the relationship between mean arterial pressure, cardiac output and total peripheral resistance (TPR). TPR depends on several factors including the length of the vessel, the viscosity of blood and the diameter of the blood vessel. The latter is the most important variable in determining resistance, changing by the fourth power of the radius, in accordance with Poiseuille's Law [1]:

$$
\begin{gathered}
\Delta p=\frac{8 Q \eta L}{\pi r^{4}}=\frac{8 \bar{v} \eta L}{r^{2}} \\
R=\frac{8 \eta L}{\pi r^{4}}
\end{gathered}
$$

where $\Delta p$ is pressure drop, $Q$ is volume flow, $R$ is vascular resistance, $\bar{v}$ is mean velocity, $\eta$ is viscosity (for air 17.1 and $19.0 \cdot 10^{-6} \mathrm{~Pa} \cdot s$ at 0 and $37{ }^{\circ} \mathrm{C}$ respectively, blood $\left.3 \cdot 10^{-3} \mathrm{~Pa} \cdot s\right), L$ is tube length, and $r$ is tube radius.

Vasodilation works to decrease TPR and blood pressure through relaxation of smooth muscle cells in the tunica media layer of large arteries and smaller arterioles. The arterioles can be vasodilated using papaverine or adenosine. 


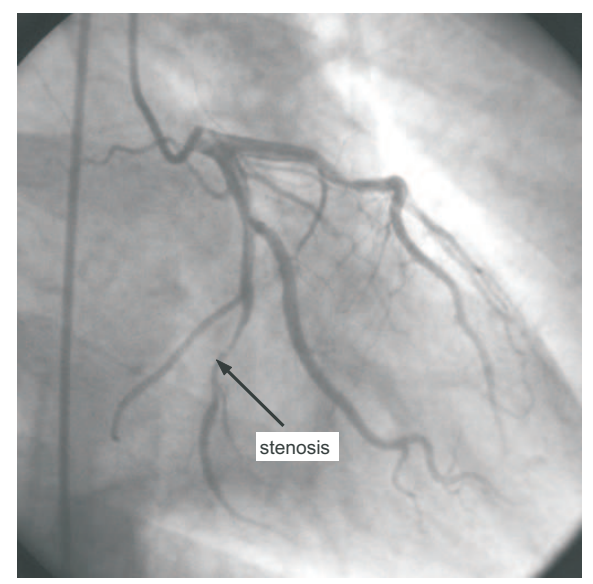

Figure 2.6: A stenosis, visualized using X-ray angiography.

\subsubsection{Flow velocity determination}

Flow velocity can be measured using the transport time of the contrast agent or bolus through the vessel. Bolus transport time methods are inherently difficult because of the pulsatile nature of blood flow, contrast diffusion and nonuniform shape of the vessel. The accuracy can be improved by gating measurement and injection of bolus and averaging of measurement. The one is in particular undesired because it requires more x-ray dose [21]. We will assume that vessels can be transformed into a straight tube with constant diameter in order to be able to estimate coronary flow velocity.

\subsubsection{Flow reserve measurement}

Stenosis severity can be determined by calculating coronary flow reserve. In figure 2.6 an example X-ray image clearly showing a stenosis is displayed. Coronary flow reserve can be calculated under the condition of maximum vasodilation. It is a simple measure that is clinical applicable. Figure 2.7 shows the concept of this measuring method [22].

Fractional flow reserve (FFR) is a pressure based measure to assess the functional severity of a coronary stenosis. The measurement procedure consist of the recording of the pressure, temperature and flow inside the coronary artery using a sensor at the tip of the catheter. A maximum heart capacity or hyperemic condition is induced using papaverine or adenosine. Then, during pull-back, the pressure is recorded. The pressure difference before and after the stenosis is denoted by the FFR.

$$
F F R=\frac{Q_{m}}{Q_{n}}=\frac{P_{d}-P_{v}}{P_{a}-P_{v}} \approx \frac{p_{d}}{p_{a}}
$$




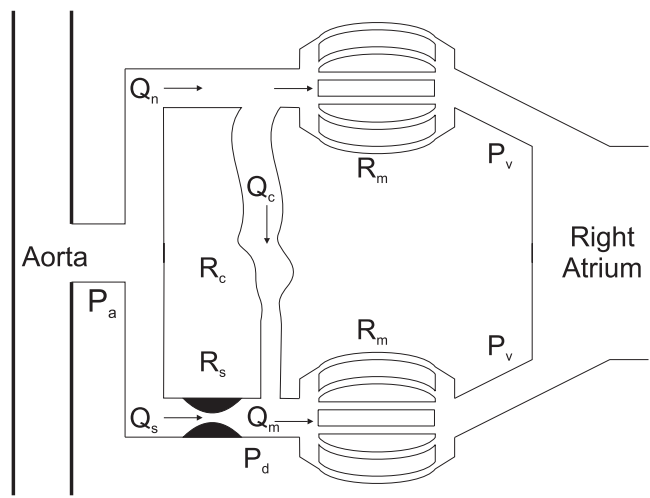

Figure 2.7: Coronary circulation model. With $P_{a}$ the arterial pressure, $P_{d}$ distal coronary pressure, $P_{v}$ venous pressure, $Q_{c}$ collateral blood flow, $Q_{s}$ blood flow through coronary artery, $R_{c}$ resistance of the collateral circulation, $R_{s}$ resistance of the stenosis, and $R_{m}$ is the myocardial resistance.

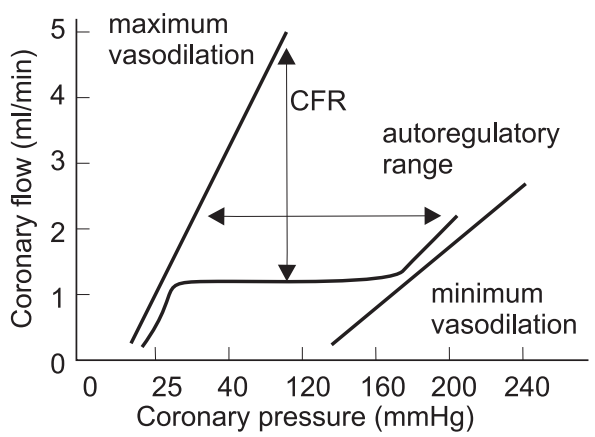

Figure 2.8: Coronary Flow Reserve (CFR) is the ratio between coronary flow in hyperemic and basal condition. Modified from Baumgart et al.[23]

The last approximation is valid if we assume $P_{v}=0 \mathrm{mmHg} . R_{m}$ is the myocardial capillary bed with a variable resistance controlled by vasodilation of the arterioles. Maximum vasodilation results in minimal resistance.

Rule of thumb is that if the FFR is smaller than 0.75 , blood flow is severely blocked and the vessel should be opened if possible.

The FFR method uses blood pressure to quantize vessel blockage. Coronary flow velocity can also be used as a measure for stenosis severity. Coronary Flow Reserve(CFR), see figure 2.8, is the ratio between coronary flow volume in hyperemic and basal condition. Calculating a ratio between the two flow volumes in basal and hyperemic condition give a number equal to the Coronary Flow Velocity Reserve (CFVR). The CFVR can be calculated using the flow velocities instead of the flow volume using Doppler wire. Doppler velocity measurement is local, this in contrast to TIMI frame counting which measures 
the time for the contrast agent to travel the entire artery giving a mean velocity. Measuring blood flow velocity in angiographic image sequences can be done using TIMI frame counting. In the next section TIMI frame counting is discussed.

\subsubsection{TIMI frame counting}

Thrombolysis in Myocardial Infarction (TIMI) flow grade is a valuable and widely used qualitative measure in angiographic trials. The TIMI grading system was proposed in 1985 by the TIMI study group [24]. The grades are defined as:

- Grade 0

Complete occlusion of the infarct-related artery.

- Grade 1

Some penetration of contrast material beyond the point of obstruction but without perfusion of the distal coronary bed.

- Grade 2

Perfusion of the entire infarct vessel into the distal but with delayed flow compared with a normal artery.

- Grade 3

Full perfusion of the infarct vessel with normal flow.

Since the flow grades are subjective and intra observer variability is quite large, an associated quantitative method called the TIMI Frame Count (TFC) is introduced. The TFC is measured by counting the number of frames between the entering of the dye in the artery until the dye reaches the end of the artery. A detailed specification about how to measure the TFC can be found in Gibson et al. [24].

The number of frames of e.g. the right coronary artery (RCA) is around 20 frames at 30 fps (at other acquisition rates a conversion factor must be applied). It appears that the TFC from the RCA is comparable to the TFC from the left circumflex artery (LCx). The TFC of the left anterior descending artery (LAD) is significantly higher because the physical length of the LAD is greater then the LCx, therefore a correction factor of 1.7 must be applied by dividing the TFC from the LAD by 1.7. This yields a CTFC (Corrected TFC) for the LAD.

When hyperemia is introduced pharmacologically using e.g. papaverine, we can measure the basal flow velocity index $\left(T F C_{b}\right)$ and the hyperemic flow velocity index $\left(T F C_{h}\right)$. Manginas et al. [25] show a significant correlation between the ratio of basal/hyperemic TFC and CFVR ratio methods with $\mathrm{r}=0.88$ and $\mathrm{p}=0.0001$, therefore we define an estimate of the coronary flow velocity reserve (CFVR):

$$
\mathrm{CFVR} \approx \frac{T F C_{b}}{T F C_{h}}
$$


The basal/hyperemic TFC ratio is called Frame Count Reserve (FCR) by Stoel et al. [26] with the conclusion that the Frame Count Reserve (FCR) and relative FCR (rFCR) can provide a good estimate of Coronary flow Velocity Reserve (CFVR) and relative Coronary flow Velocity Reserve (rCFVR) and they propose the FCR as a relative simple, fast and inexpensive angiographic method to assess the functional significance of a coronary stenosis.

\subsection{Treatments}

Intervention is traditionally performed using femoral artery access. However, access using the radial artery is increasingly applied [27]. Advantages of radial artery access are a reduced risk of bleeding, it allows the patient to walk immediately after the intervention. Also the hospital stay is shortened and the comfort for the patient is improved. Femoral artery access, however, is technically easier and has a higher success rate. Disadvantages of radial artery access are the longer learning curve, a possible increase in radiation exposure for patient and operator and coronary cannulation is more difficult.

With x-ray angiography, treatment can directly start after diagnosing a lesion. The cardiologist performing the diagnostic procedure is trained to interpret the images obtained by the angiography projection to see if there is a lesion. A stenosis can be treated by dottering, after Charles Dotter who invented this technique [28]. Dottering is a method to push the plaque into the vessel wall using a balloon brought in with a catheter. When this balloon is inflated, the balloon compresses the plaque against the arterial walls. During this inflation, blood flow is stopped. This may take a maximum of one minute, during this time the patient may feel pain in the chest because the reduction of oxygen supply to the myocardium. To prevent the vessel from returning to the original position the vessel can be supported by placing a stent, see figure 2.9, at the location of the stenosis. The vessel wall will be pushed outward which creates a wider lumen.

\subsubsection{Stents}

One stenosis treatment method is the placement of a stent. A stent is a tube which is inserted into the artery at the location of the stenosis. A balloon inflates the tube which compresses the plaque into the wall opening the artery. Stents are placed via the catheter. The placement and deployment of the stent is of significant importance, e.g. under expansion is a major contributor to instent re-stenosis and acute in-stent thrombosis [29]. Under expansion is mainly measured using Intravascular ultrasound (IVUS). Motion corrected x-ray stent visualization algorithms are developed eliminating the usage of IVUS resulting in a reduction of procedural costs. 


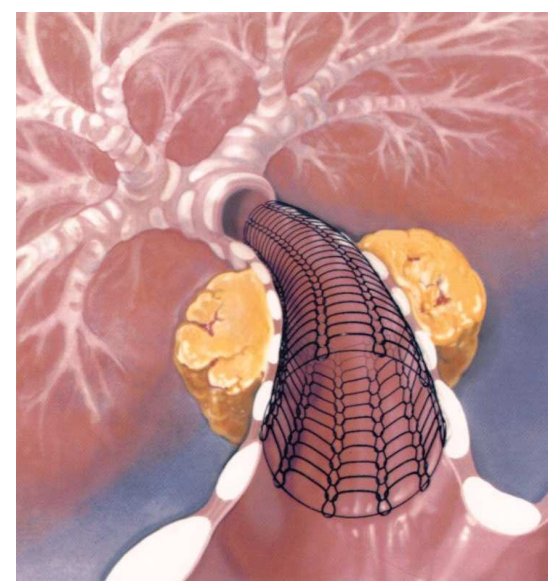

Figure 2.9: Stent placed in an artery(Image obtained from the web-site of Nagasaki Medical Center)

\subsubsection{Limitations of coronary angiography}

Coronary x-ray imaging has several advantages compared to other modalities:

- Data is available directly after assessment, with fast replay options. There is no time consuming reconstruction algorithm required.

- Spatial resolution is high, the pixel width and height is smaller than 0.5 millimeter.

- Short imaging time. Almost no setup time, patient can directly be assessed.

- High temporal resolution, allowing flow measurements in milliseconds range. Side effect is reduced motion blur due to cardiac motion.

- Small equipment, even portable equipment is available.

- Relative inexpensive.

- Relation between radiation and tissue can be used for quantitative analysis (densitometry).

- Ability to visualize coronary tree or ventricles allowing lesion detection.

Disadvantages include:

- Ionizing radiation is hazardous for people.

- The invasive procedure for inserting contrast agent requires local anesthesia and hospital admission.

- Insertion of the catheter may result in vessel wall puncture, plaque rupture and embolization.

- Iodine contrast agent can cause allergic reactions, discomfort and is nephrotoxic in high doses. 
- Images are two dimensional projections of three dimensional object. This causes ambiguities, vessel overlap and foreshortening, resulting in faulty diagnosis due to misleading arterial diameters.

- Visual inspection of angiograms introduces inter- and intra observer variabilities.

The disadvantage of intra observer variability can be reduced by using the flow map proposed in this thesis. Advantages of the flow map are:

- Retrospective flow analysis

- Stenosis assessment

- Compare flow before and after stent placement and define gain

\subsection{Quantitative coronary angiography}

\subsubsection{Introduction}

Quantitative Coronary Angiography (QCA) is the common name for the quantitative analysis of coronary arteries using x-ray angiographic images. For example: a cardiologist measures the diameter of a vessel by eye sight or more accurately with a ruler. This subjective method is prone to errors and not very reproducible $[17,10]$. Quantifying these measurements using image processing techniques results in objective values which can be used to assess functional behavior of the coronary arteries.

A method to assess arteriosclerosis is the analysis of the coronary blood flow velocity: A stenosis may cause a change in the propagation velocity of the coronary blood flow providing a good indication of the functional severity of a stenosis. The propagation of blood through a vessel can be measured using Doppler system which uses sound waves to determine the velocity of the blood. Another option is to measure the velocity using a hot-film anemometer [2]. In this thesis a method is proposed to use an x-ray image sequence with contrast enhanced coronary arteries to measure coronary flow velocity.

Two parameters of interest are the vessel diameter and the Time Density Curve (TDC). Both can be estimated from an angiographic image sequence. The TDC is defined as the contrast density $Q$ at a specific location on the vessel at a time $t$. Using the TDC, we can analyze the propagation of the contrast agent through the arteries.

Blood propagation velocity and vessel diameter are important variables in the determination of arterial diseases. In this thesis we will propose a novel method to extract propagation velocity from x-ray angiographic image sequences. The next section will describe vessel diameter properties. 


\subsubsection{Basal and hyperemic state}

In coronary x-ray angiography, calculation of the coronary flow reserve is a method to quantify the health-state of the heart by comparing the resting state or basal state with the state of maximum capacity or hyperemic state. The method is based on the measurement of velocity of the contrast agent through the coronary arteries. Using vasodilating (vessel widening) drugs arterioles can be dilated causing a higher velocity of the contrast agent [30]. By measuring the difference in flow velocity a measure for the flow velocity reserve can be calculated [26].

\subsubsection{Previous work}

Work have been done on qualitative analysis of coronary flow velocity using densitometric methods and motions correction [10, 17]. The work of Schrijver et al. aimed at the measurement of the coronary flow reserve by applying a motion correction on the coronary arteries in 2-d space. Main problem in this research were the correct tracking of landmarks on the coronary arteries. Other research groups are investigating the accuracy of modeling and reconstruction algorithms of the coronary arteries. In modeling the focus is on the field of creating a 3-d cardiac model and in reconstruction the focus is on the (tomographic) accurate reconstruction. In section 5.5 some important modeling and reconstruction algorithms are discussed. Algorithms used in quantitative analysis of 2-d images, like accurate vesseldetection and enhancement, background artifact reduction and vessel geometry estimation are still improved today and extended to the 3-d and 4-d domain to be used in MRI or other modalities.

In this thesis we will use two datasets, $A$ and $B$, of x-ray angiography assessments. Dataset $B$ has a specific focus on the measurement of coronary flow velocity in basal and hypereamic state. Therefore the dataset $B$ is used for ground truth measurements. For more details about the two datasets see appendix A.

\section{References}

[1] E. J. Topol and R. M. Califf, Textbook of cardiovascular medicine, 3rd edition, Lippincott Williams \& Wilkins, 2007.

[2] T. J. Pedley, The Fluid Mechanics of Large Blood Vessels, Cambridge University Press, 1980.

[3] L. Sherwood, Human Physiology, from cells to systems, fifth edition, Thomson Learing, Inc., 2004. 
[4] G. Baroldi, O. Mantero, and G. Scomazzoni, "The collaterals of the coronary arteries in normal and pathologic hearts," Circulation Research 4, p. 223-229, Mar. 1956.

[5] C. M. Bloor, "Functional significance of the coronary collateral circulation. a review.," The American Journal of Pathology 76, p. 561-588, Sept. 1974.

[6] A. Wahle, R. Medina, K. C. Braddy, J. M. Fox, T. M. H. Brennan, J. J. Lopez, J. D. Rossen, and M. Sonkaa, "Impact of local vessel curvature on the circumferential plaque distribution in coronary arteries," in SPIE Proceedings, 5031, 2003.

[7] A. J. Duerinckx, Coronary Magnetic Resonance Angiography, New York Springer-Verlag New York, 2002.

[8] E. A. Zerhouni, D. M. Parish, W. J. Rogers, A. Yang, and E. P. Shapiro, "Human heart: tagging with MR imaging-a method for noninvasive assessment of myocardial motion.," Radiology 169(1), p. 59, 1988.

[9] J. Park, D. Metaxas, A. A. Young, and L. Axel, "Deformable models with parameter functions for cardiac motion analysis from tagged MRI data," IEEE Transactions on Medical Imaging 15(3), p. 278-289, 1996.

[10] J. H. C. Reiber, P. W. Serruys, and C. J. Slager, Quantitative coronary and left ventricular cineangiography : methodology and clinical applications, Developments in cardiovascular medicine, Boston ; Dordrecht : Nijhoff, 1986.

[11] J. G. Rocha, C. G. J. Schabmueller, N. F. Ramon, S. Lanceros-Mendez, M. F. Costa, A. G. R. Evans, R. F. Wolffenbutten, and J. H. Correia, "Xray detector based on a bulk micromachined photodiode combined with a scintillating crystal," Journal of Micromechanical Microengineering 13, p. $45-50,2003$.

[12] J. C. Gurley, "Flat detectors and new aspects of radiation safety," Cardiology Clinics 27(3), p. 385-394, 2009.

[13] J. C. Messenger and I. P. Casserly, "Advances in contrast media and contrast injectors," Cardiology Clinics 27, p. 407-415, Aug. 2009. PMID: 19573714.

[14] W. Rutishauser, "Towards measurement of coronary blood flow in patients and its alteration by interventions," European Heart Journal 20(15), p. $1076,1999$.

[15] J. R. Womersley, "Method for the calculation of velocity, rate of flow and viscous drag in arteries when the pressure gradient is known," The journal of physiology 127(3), p. 553, 1955. 
[16] C. A. Taylor, T. J. Hughes, and C. K. Zarins, "Finite element modeling of blood flow in arteries," Computer methods in applied mechanics and engineering 158(1-2), p. 155-196, 1998.

[17] M. Schrijver, Angiographic Image Analysis to Assess the Severity of Coronary Stenosis. PhD thesis, University of Twente, 2002.

[18] G. Shechter, C. Ozturk, J. R. Resar, and E. R. McVeigh, "Respiratory motion of the heart from free breathing coronary angiograms," IEEE Transactions on Medical Imaging 23(8), 2004.

[19] R. J. Aviles, A. W. Messerli, A. T. Askari, M. S. Penn, and E. J. Topol, Introductory Guide to Cardiac Catheterization, Lippincott Williams \& Wilkins, 1 ed., Feb. 2004.

[20] J. D. Carroll, E. P. Carroll, and S. J. Chen, "Coronary angiography: The need for improvement and the barriers to adoption of new technology," Cardiology Clinics 27, p. 373-383, Aug. 2009.

[21] S. D. Shpilfoygel, R. A. Close, D. J. Valentino, and G. R. Duckwiler, "Xray videodensitometric methods for blood flow and velocity measurement: A critical review of literature," Medical Physics 27(9), p. 2008-2023, 2000.

[22] N. H. Pijls, J. A. V. Son, R. L. Kirkeeide, B. D. Bruyne, and K. L. Gould, "Experimental basis of determining maximum coronary, myocardial, and collateral blood flow by pressure measurements for assessing functional stenosis severity before and after percutaneous transluminal coronary angioplasty," Circulation 87(4), p. 1354, 1993.

[23] D. Baumgart, M. Haude, F. Liu, J. Ge, G. Goerge, and R. Erbel, "Current concepts of coronary flow reserve for clinical decision making during cardiac catheterization," American Heart Journal 136(1), p. 136-149, 1998.

[24] C. M. Gibson, C. P. Cannon, W. L. Daley, J. T. Dodge, B. Alexander, S. J. Marble, C. H. McCabe, L. Raymond, T. Fortin, and W. K. Poole, "TIMI frame count a quantitative method of assessing coronary artery flow," Circulation 93(5), p. 879-888, 1996.

[25] A. Manginas, P. Gatzov, C. Chasikidis, V. Voudris, G. Pavlides, and D. V. Cokkinos, "Estimation of coronary flow reserve using the thrombolysis in myocardial infarction (TIMI) frame count method," The American Journal of Cardiology 83, p. 1562-1565, June 1999.

[26] M. G. Stoel, F. Zijlstra, and C. A. Visser, "Frame count reserve," Circulation 107, p. 3034-3039, June 2003.

[27] I. P. Casserly and J. C. Messenger, "Technique and catheters," Cardiology Clinics 27(3), p. 417-432, 2009. 
[28] N. J. Mehta and I. A. Khan, "Cardiology's 10 greatest discoveries of the 20th century," Texas Heart Institute Journal 29(3), p. 164-171, 2002.

[29] R. K. Rogers and A. D. Michaels, "Enhanced X-Ray visualization of coronary stents: Clinical aspects," Cardiology Clinics 27, p. 467-475, Aug. 2009.

[30] C. W. Christensen, L. B. Rosen, R. A. Gal, M. Haseeb, T. A. Lassar, and S. C. Port, "Coronary vasodilator reserve. comparison of the effects of papaverine and adenosine on coronary flow, ventricular function, and myocardial metabolism," Circulation 83(1), p. 294-303, 1991. 
CHAPTER 3

\section{Flow estimation using time density curves $^{1}$}

X-ray coronary angiography is widely used to determine the presence of a stenosis. This thesis discusses an approach towards the detection of the functional severity of a stenosis using the relative velocity of the contrast agent. The velocity of the contrast is measured using the arrival time at several locations on a coronary artery. This is done by placing multiple Regions Of Interest(ROI) equally spaced on the artery. The location of these ROls varies in time because of the cardiac motion. Therefore, an artery tracing and tracking algorithm is used to estimate the location of the ROls in time. The arrival time of the contrast can be estimated by measuring the image intensity in these ROls. Using the arrival times in several ROls, a qualitative velocity can be estimated. Altering the velocity of the blood pharmacologically, by inducing hyperemic conditions, results in a qualitative change in velocity detected by the algorithm. No change in velocity may indicate a severe flow limiting stenosis.

\subsection{Introduction}

Cardiac and vascular diseases are the most common causes of death in the Netherlands, with $33 \%$ in 2004. Cardiac failure occurs when the myocardium receives insufficient oxygen. This can be caused by an obstruction in the coronary arteries, which supply the myocardium with blood. The common term for an obstruction in arteries is stenosis, see figure 3.1. A stenosis can be formed

\footnotetext{
${ }^{1}$ This chapter is published at the SPIE Medical Imaging 2006. [1]
} 


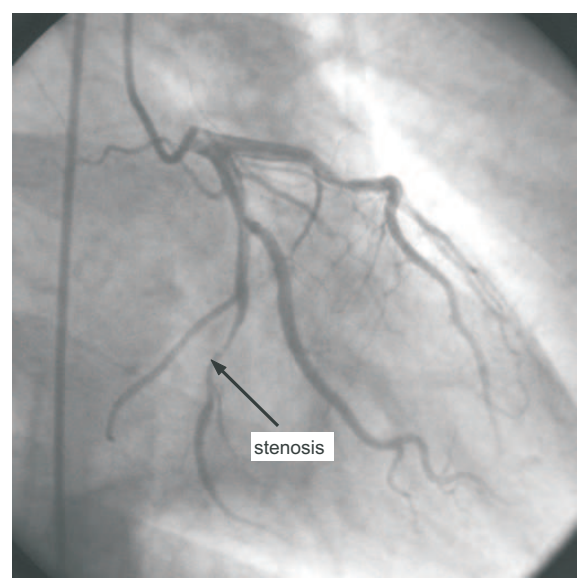

Figure 3.1: A stenosis, visualized using X-ray angiography.

by plaque accumulation on the vessel walls, so-called arteriosclerosis. The vessel lumen is narrowed and blood flow is decreased. A stenosis can be relieved using balloon angioplasty or deployment of a stent.

The common method to diagnose the presence of a stenosis is X-ray coronary angiography using the injection of a contrast material for the visualization of the coronary arteries. Other methods are intravascular ultrasound (IVUS) and Magnetic Resonance Imaging (MRI). IVUS is expensive because of the disposal after each patient scan of the ultrasound catheter. MRI is practical for diagnosing, but not for intervention. This is because of the large size of the coils which surrounds the patient completely.

The purpose of this study is to analyze X-ray angiographic images in order to determine a qualitative velocity value for the basal and hyperemic state of the heart. The concept of determining the functional severity of a stenosis using coronary flow reserve is described in Lubbers et al. [2]. Measurement of the coronary flow reserve is done by measuring blood velocity. Measurement of blood velocity by using two ROIs and detection of the arrival time of the contrast agent in the ROI, see figure 3.2. Several methods to define the transit time are proposed [3], we will use Time Density Curves (TDCs) to measure arrival times of the contrast agent. In figure 3.2, two TDCs are shown which represent the density of the contrast agent. The lower figure shows the locations of the ROIs on a vessel. Velocity can also be measured using other techniques by Sakuma et al. [4] who use MRI and by Stoel et al. [5] who use frame count reserve which has a great similarity with the technique described in this thesis. The main difference with Stoel et al. is that we are using a semi-automatic algorithm to determine the blood velocity. Main problem in the automatic detection of the blood velocity is vessel motion. 

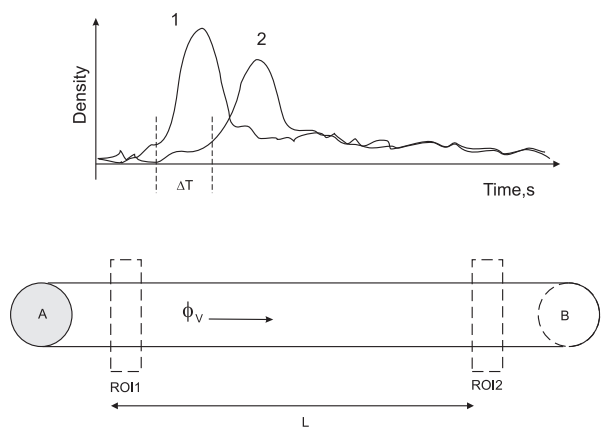

Figure 3.2: Two Time Density Curves (TDCs) are shown in the top figure, these TDCs represent the mean density level in time of the ROI at the locations in the lower figure. By measuring the time difference $\Delta t$ between two arrival times, we can calculate the blood velocity $\phi_{v}$.

In the remaining part of this thesis, X-ray angiography will be used to obtain image sequences from the coronary arteries. These sequences will be analyzed to obtain a qualitative indication of the blood velocity by using arrival time estimates of the contrast in the selected ROIs. It is also described how the blood velocity is measured and how changes in blood velocity may indicate the functional severity of stenosis.

\subsection{Methods}

This section starts with a short description of the work of Csizmadia et al. and Lubbers et al. [6, 2] who analyzed blood flow in a physical model and Schrijver et al. [7] who created an algorithm to trace and track arteries.

Csizmadia et al. [6] have modeled the behavior of blood vessels at a bifurcation, using an experimental setup with an artificial pump simulating the heart and artificial vessels. From these experiments we conclude that we can use the propagation velocity of the blood to estimate the coronary flow reserve. To do this in real practice, we have to

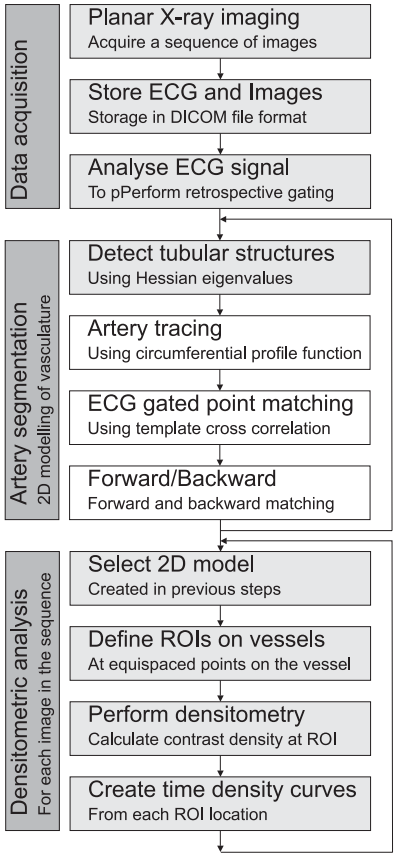

Figure 3.3: Flowchart of the artery tracing and tracking algorithm. extract the time density curve from the X-ray image sequence. In this case we used the method of Schrijver et al.[7]. 

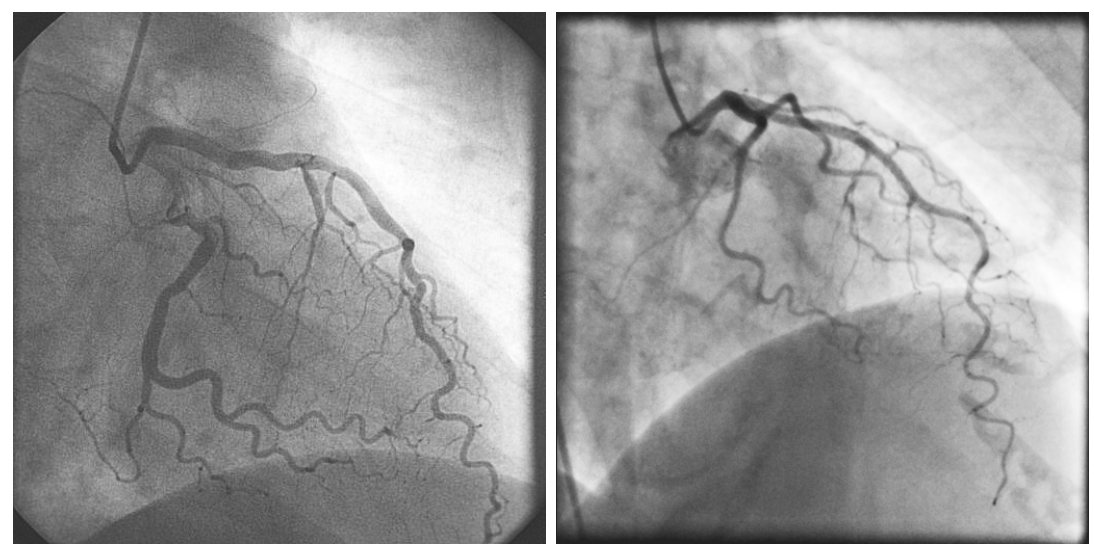

a: A typical image obtained with an in-b: A typical image obtained with a flat detensifier tube. tector.

Figure 3.4: Typical x-ray angiograms

To extract a time density curve from the X-ray image sequence, we have to determine the trajectories of points on a vessel. These trajectories are obtained by analyzing the sequence of consecutive images. The flowchart of the analysis is shown in figure 3.3. The analysis starts with the extraction of the X-ray image sequence and the ECG data from the DICOM file. The ECG data is used to determine the period of the cardiac cycle by looking at the peaks in the ECG. Just before the peak of the ECG, the heart is completely relaxed. In the relaxed state, the heart occupies the largest projected area on the intensifier screen of the X-ray imaging equipment. Arteries are clearly visible and will have minimal overlap. After automatic (or manual, if automatic detection is not satisfactory) detection of the start frame by looking at the image with the highest contrast near a QRS complex, the detection of the arteries starts. Tubular structures are detected in the start image using filter proposed by Frangi et al. [8]. This results in a two dimensional map which represents the measure of tube-similarity, also the orientation of the tubular structure is represented in a second two dimensional map containing vectors. Using this tube similarity and orientation map, arteries can be traced $[7]$.

\subsubsection{Vessel detection in 2-d}

A typical X-ray image, see figure 3.4b, passes through the vessel detector. Many vessel detection algorithms exist. Most of them are using a tubular structure detection algorithm using Hessian eigenvalues.

Tubular structure detection by using multi-scale eigenvalue based filter $[8,7]$ 
is the first step in analyzing the image sequence. The tube filter looks for tubular structures in the image with the best view on the arteries which are also well opacified by the contrast fluid. The result of the filter is a 2-d map which contains high values for a high tubular similarity in any direction and low values for low similarity.

The tube filter uses the eigenvalues from the Hessian of each point in the image to determine the tube similarity. The Hessian is defined as:

$$
H(x, y)=\left[\begin{array}{ll}
\frac{\partial^{2} I(x, y)}{\partial x^{2}} & \frac{\partial^{2} I(x, y)}{\partial x \partial y} \\
\frac{\partial^{2} I(x, y)}{\partial y \partial x} & \frac{\partial^{2} I(x, y)}{\partial y^{2}}
\end{array}\right]
$$

Using the eigenvalues of this Hessian matrix $\lambda_{1}, \lambda_{2}$ and the eigenvectors $\overrightarrow{v_{1}}, \overrightarrow{v_{2}}$, the tube similarity can be estimated. The eigenvalues and eigenvectors should be ordered according to:

$$
\left|\lambda_{1}\right| \geq\left|\lambda_{2}\right|
$$

The second derivatives of the image which are used to calculate the Hessian matrix are determined using Gaussian convolution. [8, 7]. The Gaussian kernel is calculated using a parameter $\sigma$ which represents the width of the distribution. The diameters of the vessels which should be detected are depending on this $\sigma$ parameter. For small vessels a lower bound of $\sigma=1$ can be used. For larger vessels $\sigma$ must be larger.

$$
V((x, y) ; \sigma)= \begin{cases}0 & \text { if } \lambda_{1}<0 \\ \exp \left(-\frac{R_{B}^{2}}{2 \beta_{1}^{2}}\right)\left[1-\exp \left(-\frac{S^{2}}{2 \beta_{2}^{2}}\right)\right] & \text { otherwise }\end{cases}
$$

With $R_{B}$ is a measure of the criterion $\left|\lambda_{1}\right| \gg\left|\lambda_{2}\right|$ :

$$
R_{B}=\frac{\left|\lambda_{2}\right|}{\left|\lambda_{1}\right|}
$$

And $S$ is a measure of overall curvature:

$$
S=\sqrt{\lambda_{1}^{2}+\lambda_{2}^{2}}
$$

$\beta_{1}$ and $\beta_{2}$ are scaling constants for respectively $R_{B}$ and $S$. To detect several diameters of vessels, the multi-scale vessel resemblance function is used. This function uses several $\sigma$ values and takes the maximum of all results, see equation (3.6).

$$
V(x, y)=\max _{\sigma} V((x, y) ; \sigma)
$$

Also vessel directions can be estimated using the second eigenvector:

$$
\Phi(x, y)=\angle\left[\overrightarrow{v_{2}}\left((x, y) ; \sigma_{o p t}(x, y)\right)\right] \pm k \pi
$$

Arteries can be traced by using the multi-scale vessel resemblance map $V(x, y)$ from equation (3.6) and this will be done using the algorithm explained in 3.2.1. 


\subsubsection{Tracing arteries}

For tracing arteries, or more general vessels, it is necessary to know the topology and location of the vessel in the image. Vessels are traced by starting in an initial point. This initial point may be anywhere on a vessel. To find the remaining part of the vessel, a circumferential profile function is used [7]. This function corresponds to the intensities in the image which lie on a circle, with a certain radius, using the initial point as the center. We use a radius corresponding to 16 pixels. Vessels show up dark in the X-ray images. By finding the local minima of this profile, the vessel direction can be estimated. All local minima are represented by a weight, indicating the likelihood of a vessel, and direction. The most likely direction has the highest weight. These weights are sorted and the direction of the highest weight is chosen as the direction of the vessel. The location of this local minimum is used as the initial point in the algorithm and the next iteration can start. All directions with weights above a empirical defined threshold, are used in this algorithm. This makes it possible to find bifurcations.

\subsubsection{Tracking arteries}

The traces of the vessels are divided into landmarks at the start and end of a vessel and at the bifurcation points. These landmarks are used to select a square region around the landmark which is used for tracking the position of the artery in time. The new position of the artery is obtained using template matching. For each landmark this algorithm looks for the new position of the landmark in a search window close to the initial landmark, the size of this search window depends on the frame rate of the image sequence. A sequence with a higher frame rate can use a smaller area because movements of the arterial tree appear smaller. When the similarity measure is too low, the user is asked to indicate the position of the artery. This manual correction is necessary to prevent propagation of tracking errors. Also manual correction must be applied in frames in the pre-contrast phase of the X-ray frame sequence. At the end of this phase, all landmarks have a trajectory which indicate the position of the vessel in time, see figure 3.5.

\subsubsection{Interpolation}

We have to know the location of the vessel before being able to place regions of interest. Landmark information only is insufficient, because of the low number of landmark points. Therefore, landmarks together with the image information is used to interpolate between landmarks. This is done using a minimal cost path algorithm. The lines obtained by this interpolation are subdivided in equidistant points, these tracepoints can be selected as the center point of a ROI. 


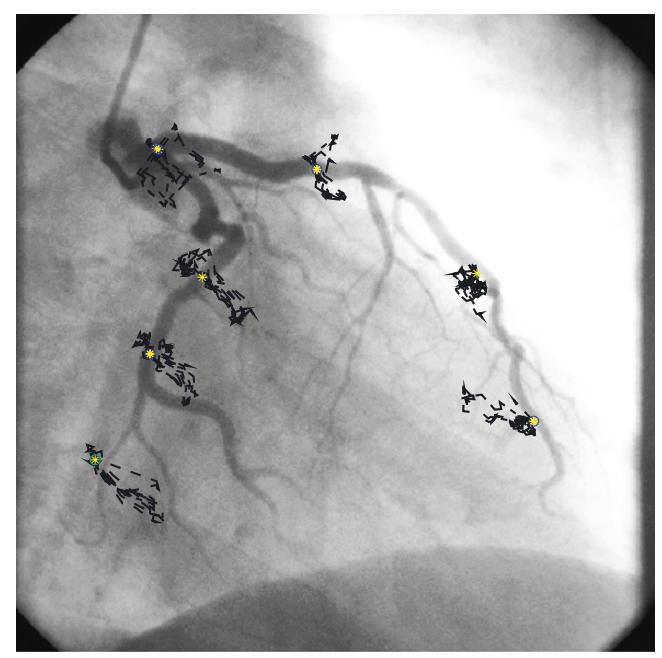

Figure 3.5: The motion trajectories of several tracepoints in consecutive images.

\subsection{TDC extraction}

\subsubsection{Introduction}

The previous section described the methods used to extract the location of the vessel and the trajectory in time from the images. In this section we will use this information to extract Time Density Curves (TDCs) from the data. For this we assume that the contrast agent behaves like a step function, in reality the percentage of mixing with the blood will determine the profile of the TDC. First we will address the expected accuracy of the velocity estimation due to the limited frame rate of the imaging equipment.

\subsubsection{Frame rate}

The frame rate is an important factor in the tracking algorithm. If the frame rate is too low, time information about the location of the arteries is missing. It will be more difficult to find the artery because the distance in a longer period of time is larger. Tracking a large distance introduces more errors because the search space becomes larger. Also the frame rate is of importance in the determination of the blood velocity. The accuracy of this measurement is related to the frame rate.

The blood velocity in the LAD coronary artery is between 5 and $20 \mathrm{~cm} / \mathrm{s}$ in the basal state and between 10 and $60 \mathrm{~cm} / \mathrm{s}$ in the hyperemic state using the vasodilator dipyradimole [4]. If we approximate the space between tracepoints in the coronary angiogram at $1 \mathrm{~cm}$, then a frame rate of 25 frames/second is sufficient to detect a velocity, although not very accurate. 


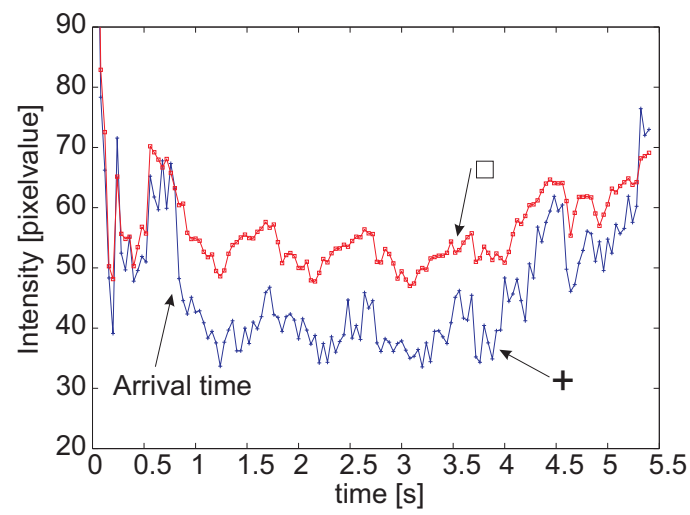

Figure 3.6: Typical TDCs measured in a [3×3] ROI (marked with a + ) and a circular ROI with a diameter of 30 pixels (marked with a $\square$ ). The [3×3] ROI TDC is noisier but also shows sharper edges which can be used to detect the arrival time of the contrast agent.

\subsubsection{Time density curve extraction}

For estimation of the blood velocity in a vessel, regions of interest (ROIs) must be placed on the vessel. These ROIs are placed equidistantly on the vessel. More than two ROIs are necessary in order to find a regression line which represents the blood velocity. The user can select any of the tracepoints as the center point of a ROI. A Time Density Curve, see figure $3.6^{2}$, can be created at each tracepoint location on a vessel. This curve is created by taking the mean value of the selected ROI at each frame of the image sequence. Extrapolation of the location of the ROI in the pre-contrast stage using the contrast stage is necessary to obtain an estimate of all ROI locations during the measurement cycle. This extrapolation is done using correctly traced frames and the period of the cardiac cycle estimated from the ECG. When a frame has not enough contrast to correctly track the arteries, then a trace of the frame with an index $f_{i}$ will be used:

$$
f_{i}=f_{i}+n N_{c}
$$

where $n \in \mathbb{N}$ and $N_{c}$ is the number of frames in a cardiac cycle.

\subsubsection{Time difference}

To measure the velocity of the blood, we have to estimate the time difference between TDCs. This can be done by locating the arrival time in the TDC,

\footnotetext{
${ }^{2}$ Notice that the TDCs plotted in figure 3.6 are inverted compared to the expected TDCs in figure 3.2 , this is because we are measuring intensity instead of density. The intensity is related to the density according to Lambert-Beer's law, but this relation is not important in determining the arrival time
} 
by locating the center of gravity or measure the time difference between two TDCs using correlation methods.

Several methods to estimate the arrival time have been tested. Correlation between two TDCs could result in a phase shift of the maximum peak, which should indicate the delay between two TDCs. An other method is mean square estimation. This method takes the first trace and tries to minimize the error between another trace while shifting the other trace back in time. The shift distance with the smallest error should represent the delay between two traces. The correlation method does not work as expected. Main cause is the sensitivity of noise, both TDCs contain a high amount of noise. The other method, minimizing the error between two traces, performs well in simulations with artificial data, but fails significant when noise is added to this data. The best method we have tested is filtering using a matched filter.

\subsubsection{Low Noise TDC}

General low pass filtering of the signal is not an option, low pass filtering distorts the signal and results in lower accurate estimates of the arrival time. Low pass filtering is performed using low pass filters and with a large circular ROI. A large circular ROI (diameter $D=30$ pixels) covers a larger area of the blood vessel, which causes a better performance of the algorithms compared to the normal low pass filter. But with a small ROI the results are much better. So we will use the $[3 \times 3]$ ROI for extracting our TDCs. This $[3 \times 3]$ ROI is centered in the middle of the vessel where the density of the contrast fluid is high and the intensity level in the image is low. By enlarging the ROI, more pixels are covered, but these pixels have a lower density. This results in increased inaccuracy. The best algorithm so far tested is the algorithm in which a matched filter is used to determine the arrival time.

The TDC is used to estimate the arrival time of the contrast. In our case, the X-ray beam is attenuated corresponding the density of the contrast agent, therefore a detection from high intensity to low intensity is made. For this detection a standard matched filter is used with the following properties:

$$
\begin{array}{r}
y(n)=\sum_{k=-N_{\text {before }}}^{N_{\text {after }}} x(k) h(n-k) \\
h(n)=\left\{\begin{array}{rr}
1 & -N_{\text {before }} \leq n \leq 0 \\
-1 & 0<n \leq N_{\text {after }} \\
0 & \text { otherwise }
\end{array}\right.
\end{array}
$$

where $y(n)$ is defined as the matched filter convolution, $h(n)$ is the impulse response of the matched filter. The parameters $N_{\text {before }}$ and $N_{\text {after }}$ represent the 'clear' number of frames before injection of contrast agent and the number of frames during the contrast injection respectively. The result is scaled and normalized to enhance the visualization of the peaks in figure $3.8 \mathrm{~b}$ and $3.8 \mathrm{a}$. 
The type of matched filter is important, we have to deal with an unknown signal from which we only know that the signal starts high and becomes low at a certain point and then slowly increases to the high value. The high part is the period where no contrast is available. The low part is the period where the contrast arrives, this can be gradually with an unknown time interval depending on the dilution of the contrast in the blood. The third part is the washout phase, after the stop of the injection of the contrast, a time interval is needed to washout the indicator. Because of these not accurately know parameters we take empirically found values of $N_{\text {before }}=60$ and $N_{\text {after }}=160$ with image sequences of $25 \mathrm{fps}$ which are used in the experiments described in this thesis .

\subsubsection{Procedure}

Angiography is performed using a Philips Integris (25 fps). Hyperemic conditions are induced by using a vasodilating drug, in this case papaverine. In healthy persons, dilation causes a higher blood velocity in the arteries compared to the basal condition. About 3 minutes after papaverine injection, the blood velocity is higher. The images used in this thesis are made using manual injection of the contrast agent which is injected at a higher rate in the postpapaverine study to obtain a higher contrast. The higher propagation can be measured using our algorithm, as will be shown experimentally. The artery tracing and tracking algorithm must be performed on (1) the pre-papaverine study (basal condition) and (2) in the post-papaverine study (hyperemic condition).

\subsection{Experiments}

Our experiments exist of the analysis of X-ray image sequences of five patients which are visually selected on traceability by looking at the contrast of the image and the overlap of the vessels. For good tracing no or minimal vessel overlap and high contrast is required. They all contain the Left Anterior Descending coronary artery (LAD). The algorithm is applied on these sequences after manually selecting a good start frame. The tracing and tracking of the sequence is automatically up to $98 \%$ of the cases. The remaining $2 \%$ of all landmarks have to be matched manually. An indication of the total number of landmarks is 2000-4000 depending on the size of the tracked vasculature. After applying the algorithm to the sequence, the tracing and tracking information is used to extract the TDCs. The ROIs are placed on an artery as shown in figure 3.7. From all 10 image sequences, 5 of the basal and 5 of the hyperemic state, the velocity is estimated. The results will be presented in the next section. 


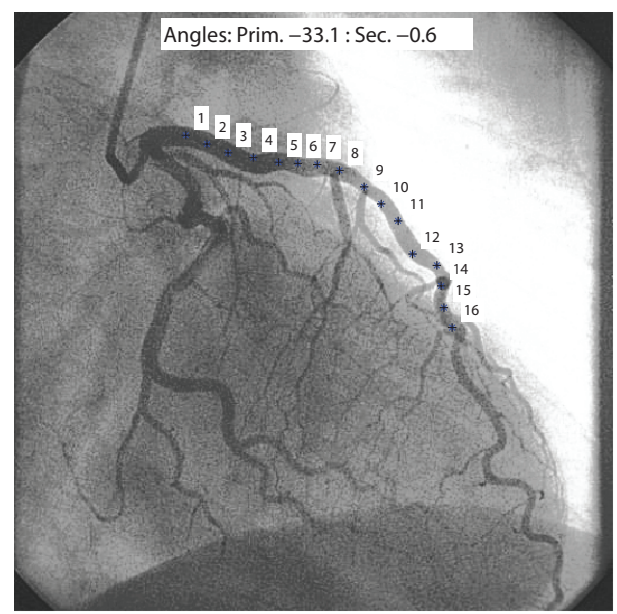

Figure 3.7: One image of the sequence indicating the locations of the ROIs

\subsection{Results}

This section describes the results obtained by the placement of several ROIs on a single vessel. Figure 3.7 shows the location of the ROIs that are used to measure the contrast density.

The result of the matched filter is plotted in the lower graphs of the previous two figures. The vertical line in the upper plot shows the maximum found in the matched filter graph. This is the best match with the data, which corresponds to the entry time of the contrast into the ROI. Using these entry times and plotting the ROI number against time as can be seen in the figures $3.9 \mathrm{a}$ and $3.9 \mathrm{~b}$ for respectively the analysis in basal and hyperemic conditions, we can perform linear regression analysis of the entry times. As can be seen in the figures the frame time in which the contrast enters the next ROI in the tracing of the basal condition is $a x+b$ with $a=1.3$ and $b=16 ; x$ defines the spacing between the ROIs which is assumed to be $1 \mathrm{~cm}$. The constant $b$ refers to the number of frames which that passed before the detection of the contrast agent in the first frame. In the figure of the results in the hyperemic condition, we can see that the velocity is increased to $a=0.6$ and the number of frames passed is $b=7.4$. This constant $b$ is highly depending on the time of the acquisition start and the injection of the contrast. Because this is done manually, this value is not very useful. The other two values, $a=1.3$ and $a=0.6$ indicate the change of the propagation velocity in hyperemic conditions. The velocity is increased with the ratio

$$
C F V R=\frac{v_{\text {basal }}}{v_{\text {hyperemic }}}=\frac{1.3}{0.6}=2.16
$$

in which CFVR is defined as the Coronary Flow Velocity Reserve (CFVR). The 

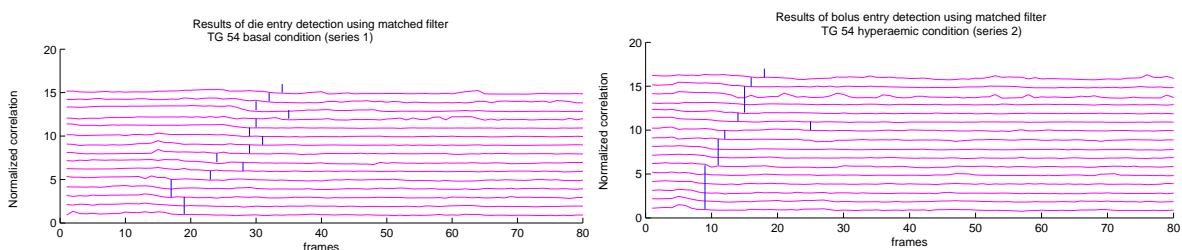

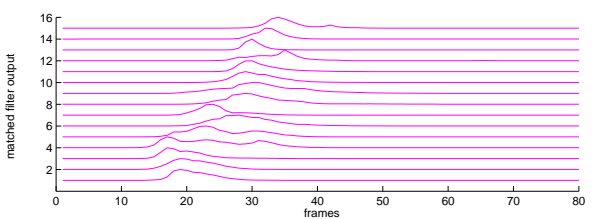

a: Basal condition

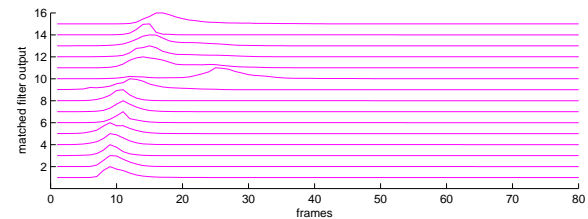

b: Hyperemic condition

Figure 3.8: Times of entrance of the ROI measured in two runs, the upper graphs shows the TDCs with the detected arrival time shown as a vertical bar and the figure below shows the (normalized) output of the matched filter. The locations of the ROIs belonging to the TDCs in this figure are shown in figure 3.7.
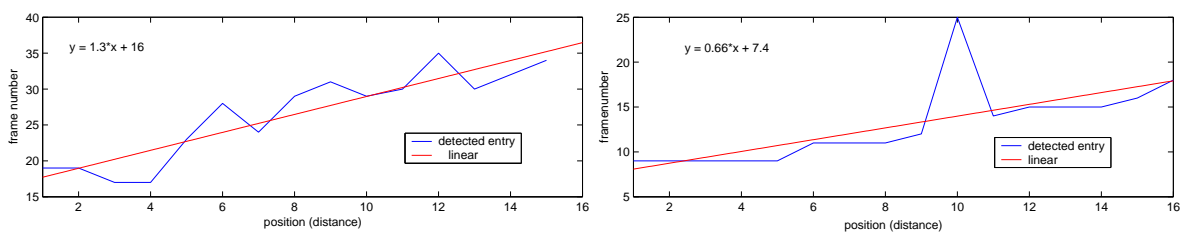

residuals

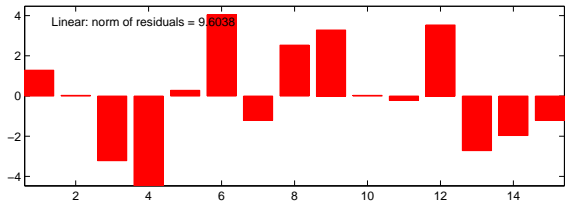

a: Basal condition

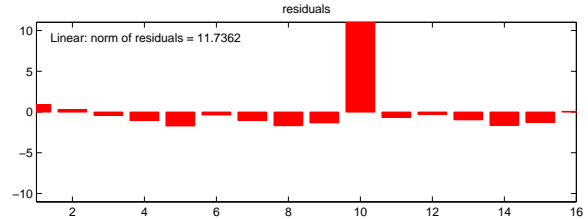

b: Hyperemic condition

Figure 3.9: Linearized appearance of the contrast in time and distance, the graphs of the hyperemic condition shows a faster propagation time, this is indicated by the coefficient $a=1.3$ compared to $a=0.66$. The coefficient $b$ is an indication of the start of the contrast injection. 
CFVR can be used in this case as the change of the cardiac rhythm $\left(\Delta F_{\text {heart }}\right)$ in the basal and hyperemic state is not significant:

$$
\Delta F_{\text {heart }}=\frac{n_{b}\left(Q R S_{5}\right)-n_{h}\left(Q R S_{1}\right)}{n_{b}\left(Q R S_{5}\right)-n_{h}\left(Q R S_{1}\right)}=\frac{113-20}{117-30}=1.06
$$

This value is measured by taking the frame number (n) at the peak of QRS complex number 1 and QRS complex number 5. Hemodynamic analysis is not taken into account in this experiment, analysis of the effect of hemodynamics [9] on the results should be investigated in the future.

In the previous example, we have shown how we estimate the velocity of the blood. The next section will give a quantization approximation of the data. The size of the intensifier screen is $170 \mathrm{~mm}$ in diameter sampled with a resolution of $[512 \times 512]$ pixels. The position of the detector to the patient is not available from the DICOM data of the Philips Integris used to acquire the images in this example, but according to the information in DICOM files of the Siemens Axiom Artis, we can assume a position of the source to the patient of around $750 \mathrm{~mm}$. From this information we can estimate that the physical width of each pixel in the image is approximately $0.3 \mathrm{~mm}$. With a trace point distance of 30 pixels, this results in approximately $1 \mathrm{~cm}$ between tracepoints.

\subsection{Discussion and conclusion}

\subsubsection{Noise}

The TDCs contain noise. This noise has several origins: (1) Quantum and other noise in the X-ray image, (2) tracing noise, the center of the vessel is not detected correctly, (3) tracking noise, template matching errors can occur causing a wrong location of the ROI which causes noise in the TDC, (4) Opacification level of the vessel, the contrast is not completely replacing the blood which causes opacification noise in the TDC. A higher blood velocity results in a lower image contrast when injecting the same amount of contrast as in the pre-papaverine study.

A low opacification level also introduces noise in the tracing/tracking algorithm, this is because the matched filter algorithm fails to detect the correct arrival time if the artery is not opacified long enough. A minimum amount of opacification is required otherwise the algorithm returns maxima at later positions in time because the opacification level becomes pulsatile. Filtering of this noise is only possible if there is enough spatial resolution. Because of the low spatial resolution (15-30 frames/sec), filtering, for example low pass filtering, is not a very good method to obtain better results. 


\begin{tabular}{|l|l|l|c|c|}
\hline Study & series & type & velocity $[\mathrm{cm} / \mathrm{s}]$ & deviation $[\mathrm{cm} / \mathrm{s}]$ \\
\hline NA75 & 0001 & basal & 50 & 14 \\
\hline NA75 & 0003 & hyperemic & 28 & 7 \\
\hline TG54 & 0001 & basal & 20 & 4 \\
\hline TG54 & 0002 & hyperemic & 48 & 11 \\
\hline MJS0 & 0011 & basal & 15 & 4 \\
\hline MJS0 & 0013 & hyperemic & 182 & 46 \\
\hline MDP1 & 0013 & basal LAD & 3 & 3 \\
\hline MDP1 & 0014 & hyperemic LAD & 41 & 12 \\
\hline MDP1 & 0013 & basal LCx & 19 & 5 \\
\hline MDP1 & 0014 & hyperemic LCx & 77 & 41 \\
\hline
\end{tabular}

Table 3.1: Results of ten measurements using the the complete algorithm path.

\subsubsection{Comparison with previous studies}

If we assume that the spacing between tracepoints is $1 \mathrm{~cm}$ then we can quantify the velocity of the blood and compare the results with the results of Sakuma et al. [4] In our measurements, see table 3.1, we detect a mean velocity of $21 \mathrm{~cm} / \mathrm{sec}$ \pm 6 using a $25 \mathrm{fps}$ image sequence in the basal state. In the hyperemic state a mean velocity of $72 \mathrm{~cm} / \mathrm{sec} \pm 17$ is measured. ${ }^{3}$ In the article of Sakuma et al. [4] values of respectively $14.8 \mathrm{~cm} / \mathrm{sec} \pm 1.9$ and $46.3 \mathrm{~cm} / \mathrm{sec} \pm 10.2$ are detected using the vasodilator dipyradimole and measuring the LAD. It is impossible to compare the results exactly because of the strong variation in patient data, but similar results are also obtained in the article by Stoel et al. [5]. The difference in the technique of Stoel et al. and our technique is that we are using an a semi-automatic image processing technique to detect the blood velocity.

\subsubsection{Towards the detection of the functional severity of stenosis}

If we can limit the amount of noise, we can start to look at the blood velocity differences before and after stenosis treatment. The estimated velocity in this thesis uses a linear fit of 15 points equispaced on a vessel. To measure before and after a stenosis, only 2 or 3 points are available for measurements. In the current setup, the noise disturbs the detection of the correct arrival time. Which does not allow a three point measurement for the velocity.

Therefore we propose another method: We can obtain information about the blood velocity by extracting a flow-map, see figure 3.10. This can be done by using the centerline of a vessel and get the pixel values of this centerline from

\footnotetext{
${ }^{3}$ Notice that in the NA75 study, the hyperemic condition has a lower blood propagation compared to the basal condition. This is a false measurement due to the low dilution of the contrast agent in the blood. This causes false predictions of arrival times.
} 


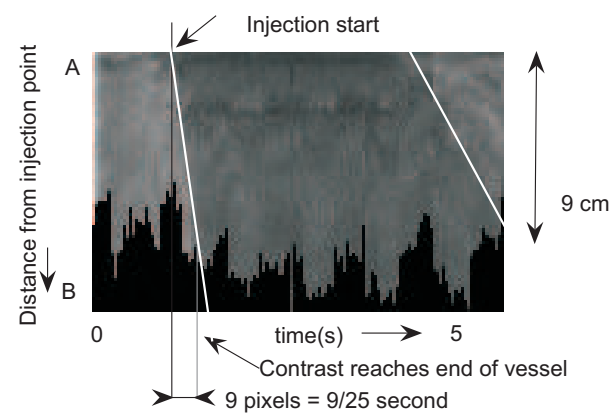

Figure 3.10: Flow-map of an image sequence. Each frame is represented by a position on the $\mathrm{x}$-axis (frames numbers are converted to seconds using a division by $25 \mathrm{fps}$ ). The $\mathrm{y}$-axis shows the pixel values of the centerline of a vessel starting from $\mathrm{A}$ to $\mathrm{B}$ in which $\mathrm{A}$ is the injection point of the contrast agent. The length of the A-B section varies because of the movement of the vessel (the length of the vessel changes in time). Two white lines are superimposed indicating the speed of the flow (left line injection flow and right line washout flow).

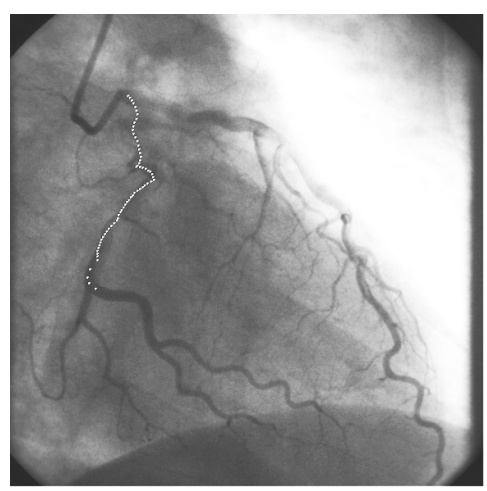

Figure 3.11: The flow-map images are produced using the centerline of the vessel. This centerline is shown as a series of dots starting at label A and ending at label B. The pixel values of the image at the vessel centerline are used to form a horizontal row in the flow-map.

each frame. This centerline is represented as a horizontal row in the flow-map, this row varies in length because the visible vessel length also changes in time. The vessel and centerline used for this image is shown in figure 3.11. These figures give a good overview of the blood flow through the vessel which may be useful in future research. If we look at the flow-map and draw a line on the border of the with/without contrast agent and quantify the slope of this line by estimating the length of the vessel to be $9 \mathrm{~cm}$. In addition, we estimate the time required by the contrast agent to reach the end of the vessel to be $9 / 25$ second. Using these estimates, the blood velocity will be $25 \mathrm{~cm} / \mathrm{s}$. Comparing 
this value with previous results (see table 3.1 TG54 series 1) a difference of 5 $\mathrm{cm} / \mathrm{s}$ exists. When this line detection and approximation of the vessel length is automated, then the flow-map method is expected to be more accurate.

Future research may concentrate on the analysis of the flow-map images obtained by our algorithms and optimization of the algorithms to minimize manual intervention.

\section{References}

[1] G. A. ten Brinke, C. H. Slump, and C. J. Storm, "Digital densitometric determination of clinical relative coronary flow distributions," in Proceedings of the SPIE, 6143, International Society for Optical Engineering, 2006.

[2] A. P. G. Lubbers, C. H. Slump, and C. J. Storm, "Digital densitometric determination of relative coronary flow distributions," in Proceedings of the SPIE Vol. 1905, Biomedical Image Processing and Biomedical Visualization, p. 270-280, July 1993.

[3] S. D. Shpilfoygel, R. A. Close, D. J. Valentino, and G. R. Duckwiler, "X-ray videodensitometric methods for blood flow and velocity measurement: A critical review of literature," Medical Physics 27(9), p. 2008-2023, 2000.

[4] H. Sakuma, L. M. Blake, T. M. Amidon, D. H. Szolar, A. P. Furber, M. A. Bernstein, T. K. F. Foo, and C. B. Higgins, "Coronary flow reserve: Noninvasive measurement in humans with Breath-Hold velocity-encoded cine MR imaging," Cardiac Radiology 198, p. 745-750, 1996.

[5] M. G. Stoel, F. Zijlstra, and C. A. Visser, "Frame count reserve," Circulation 107, p. 3034-3039, June 2003.

[6] N. P. Csizmadia, M. Schrijver, C. H. Slump, and A. P. G. Lubbers, "Digital densitometric determination of relative coronary fllow distributions," Medical and Biological engineering and computing 39, p. 303-309, Feb. 2001.

[7] M. Schrijver, Angiographic Image Analysis to Assess the Severity of Coronary Stenosis. PhD thesis, University of Twente, 2002.

[8] A. F. Frangi, W. J. Niessen, R. M. Hoogeveen, T. van Walsum, and M. A. Viergever, "Model-based quantitation of 3-D magnetic resonance angiographic images," IEEE Transactions on Medical Imaging 18(10), p. $946-56,1999$.

[9] A. J. Duerinckx, Coronary Magnetic Resonance Angiography, New York Springer-Verlag New York, 2002. 


\section{CHAPTER 4}

\section{Flow estimation using a flow map ${ }^{1}$}

Coronary stenosis are often assessed using planar X-ray angiography while injecting contrast agent. The data obtained during the intervention can be used to assess coronary flow by tracking the contrast agent. The contrast flow can be used to estimate the functional behavior of the coronary arteries using TIMI frame counting. In this thesis we propose and evaluate a method towards the automation of TIMI frame counting. The method creates a two dimensional map of the contrast agent in which the opacification of the vessel centerline is plotted against time. This map is used to find the velocity of the contrast agent and subsequently the TIMI frame count. The vessel centerline is found using the Fast Marching Method to find the lowest cost path between the catheter point and the manually selected endpoint of the vessel. Results from 22 patients show that the algorithm is able to estimate TFC correctly in 13 of 22 cases within the intra observer error range of \pm 5 frames compared to manual TFC.

\subsection{Introduction}

Thrombolysis in Myocardial Infarction(TIMI) flow grade is a valuable and widely used qualitative measure in angiographic trials. The TIMI grading system was proposed in 1985 by the TIMI study group [1]. The grades are as follows: 0: Complete occlusion of the infarct-related artery, 1: Some penetration of contrast material beyond the point of obstruction but without perfusion

\footnotetext{
${ }^{1}$ This chapter is presented at the SPIE Medical Imaging 2008.
} 
of the distal coronary bed, 2: Perfusion of the entire infarct vessel into the distal but with delayed flow compared with a normal artery and 3: Full perfusion of the infarct vessel with normal flow. Since the flow grades are subjective and intra observer variability can be large, Gibson et al. [1] introduced an associated quantitative method called the TIMI Frame Count (TFC). The TFC is measured by counting the number of frames between the entering of the dye in the artery until the dye reaches the end of the artery. The TFC of e.g. the right coronary artery (RCA) is around 20 frames at 30 fps (at other acquisition rates a conversion factor must be applied) for healthy vessels and it increases to infinity (total occlusion) in diseased vessels. It appears that the TFC from the RCA is comparable to the TFC from the left circumflex artery (LCx). The TFC of the left anterior descending artery (LAD) is significantly higher, therefore a correction factor of 1.7 must be applied yielding a CTFC (Corrected TFC) for the LAD. Bickel et al. [2] confirmed that the semi-quantitative TFC method is superior to the TIMI flow grading method. The time of injection of the contrast agent only influences the TFC by 2 frames [3]. When hyperemia is introduced pharmacologically using e.g. papaverine, we can measure the TFC in basal state and in hyperemic state. Division of the basal TFC (basal flow velocity index) by the hyperemic TFC (hyperemic flow velocity index) results in an estimate of the coronary flow velocity reserve. Stoel et al. [4] conclude that the Frame Count Reserve (FCR) and the relative FCR (rFCR) can provide a good estimate of Coronary flow Velocity Reserve (CVR) and relative Coronary flow Velocity Reserve (rCVR) and they propose the FCR as a relative simple, fast and inexpensive angiographic method to assess functional significance of coronary stenosis. Although Computed Tomography Angiography (CTA) is becoming more popular, planar X-ray angiography is still the main modality for coronary intervention. Planar X-ray imaging is also the best modality for measuring contrast flow using TIMI frame counting [5]. In this thesis, we propose a method which uses 2-d X-ray images to measure the contrast flow based on a similar principle as TIMI frame counting.

\subsection{Method}

\subsubsection{Preprocessing}

A planar X-ray image sequence, $I(n), n=[1 . . N]$, containing a clear projection of the vessel before, during and after the injection of the contrast agent is required. The recorded ECG signal is used to estimate how many frames are recorded in one cardiac cycle. All images are contrast enhanced using log-subtraction of the background. The background, $I_{\text {dilated, }}$ is created by morphologically dilation with a 2-d Gaussian structuring element of size $[15 \times$ 15] pixels:

$$
I_{c}(n)=\log (I(n))-\log \left(I_{\text {dilated }}(n)\right)
$$


In all images the catheter endpoint and the vessel endpoint are manually selected. Automatic algorithms are proposed [6], but in our case we want to make sure that the startpoint and endpoint of the vessel is correct.

\subsubsection{Contrast flow map}

A contrast flow map, $C F M(s, n)$, is created from the selected vessel. The flow map consist of $n=[1 . . N]$ rows, wth $n$ the frame in the image sequence. The spatial position $s$ is the normalized lengt $s=[0 . .1]$ of the vessel from the start $s=0$ to the endpoint $s=1$. A typical flowmap is show in figure 4.1 , the temporal data $n$ is plotted along the vertical axis and the spatial $s$ is plotted along the horizontal axis. Each row represents the opacity at the centerline of the vessel in which the vessel is parametrised by $s=[0 . .1]$. The flowmap is created using the centerline of the vessel by tracking it throughout the image sequence. The centerline is found by finding the fastest path along a vessel from the manually annotated catheterpoint and the endpoint of the vessel.

Flow map. Dataset: 7

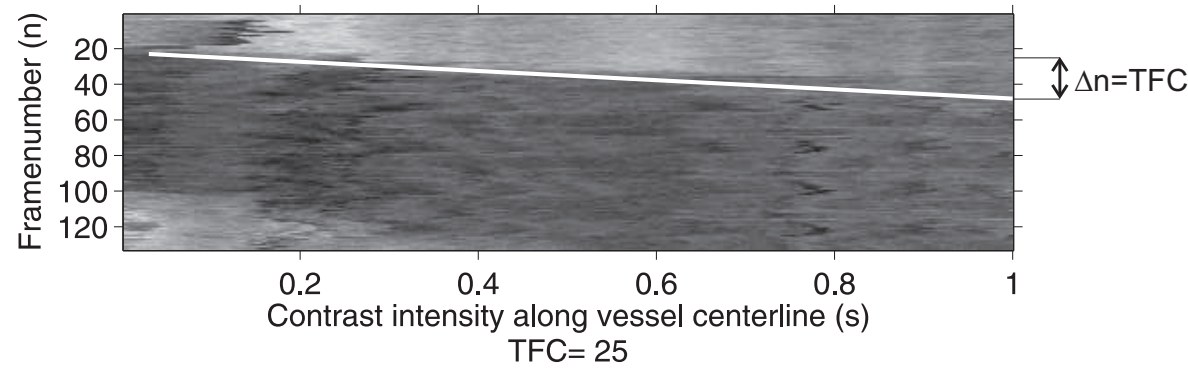

Figure 4.1: Flowmap for dataset 7.

\subsubsection{Vessel tracing}

The vessel centerline is found using the Fast Marching Method (FMM)[7]. The FMM is typically used to build a map containing the travel time between the startpoint and all other points using a speed function. The fastest path from the starting point to an end point can be found and is defined by $x(s)$ and $y(s)$ in which $s$ runs from the start of the vessel to the endpoint. $x(s)$ and $y(s)$ are the locations of the vessel in the image at position $s$. The FMM solves the Eikonal equation:

$$
\|\nabla T(x, y)\| F(x, y)=1
$$

In which $T(x, y)$ is the arrival time of the front and $F(x, y)$ is the speed function. The speed function used as input for the Fast Marching method is 

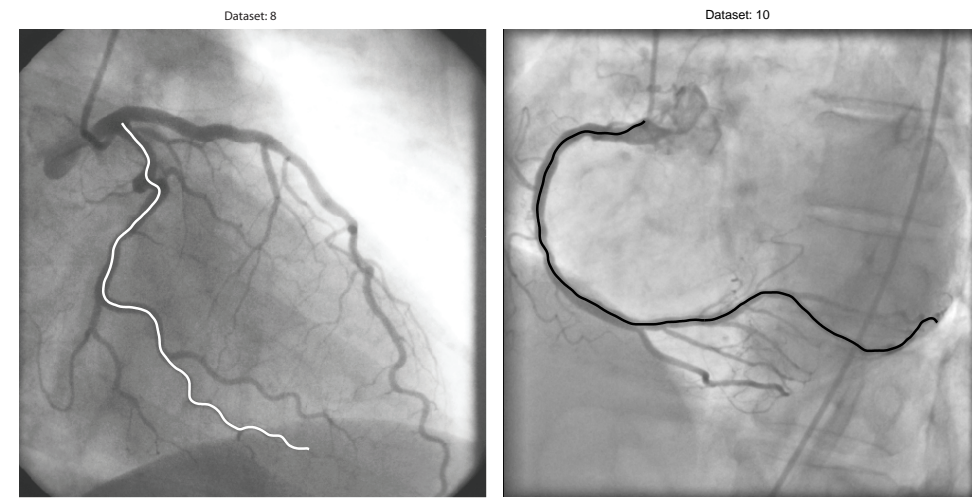

Figure 4.2: A typical vessel centerline found using the Fast Marching method. In the displayed cases of the LCA (left) and the RCA (right) only the start and end point is manually selected.

based on the contrast enhanced image $I_{c}(x, y)$ :

$$
F(x, y)=\left(1-\left(I_{c} \otimes G_{\sigma}\right)\right)^{\gamma}
$$

Where the operator $\otimes G_{\sigma}$ is defined as Gaussian convolution with standard deviation $\sigma$. The image data is inverted so that low intensity means higher speed. Then the discrimination between low and high intensity is increased using a power of $\gamma$. In this research we have used values of $\sigma=1.5$ and $\gamma=10$. The fastest route from the startpoint to the endpoint is used as vessel centerline. A typical centerline found by this algorithm is shown in figure 4.2. The contrast enhanced image $I_{c}$ is used to find the pixel values on the vessel centerline. The pre-contrast agent frames do not have a clear vessel centerline. For these cases we use vessel centerlines found in the frame $n+N_{p}$, in which the constant $N_{p}$ is defined as the number of frames between two R tops of the ECG. This centerline is written as $\operatorname{trace}\left(I_{c}(n), s\right)$. The data of the rows in the flow map are defined as:

$$
C F M(n, s)=\min \left(\operatorname{trace}\left(I_{c}(n), s\right)\right)
$$

With $s$ is defined as the normalized vessel length in the range $s=[0 . .1]$. Pixel values lower than the pixel values measured in the previous $N_{p}$ frame are used in the contrast flow map $C F M(s, n)$. The fastest path through a vessel in one frame is not always of the same length as in another frame. Therefore the whole line of pixel data is scaled using linear interpolation to the length of the contrast flow map. 


\subsubsection{Contrast flow map analyis}

The DC offset is removed from all spatial components using the mean value in the temporal direction:

$$
C F M_{d c}(n, s)=C F M(n, s)-\frac{1}{N} \sum_{n=1 . . N} C F M(n, s) \quad s=[0 . .1]
$$

Next we calculate a threshold using a 256 bins histogram from $C F M_{d c}$. In general, the maximum peak in the histogram belongs to the contrast agent (dark) values. We assume that we can seperate the opacified arteries from the transparent arteries using the value of the maximum peak position in the histogram $+1 / 8$ as threshold. $C F M_{d c}$ is smoothed in temporal direction using a Gaussian with $\sigma=5$. After this, the threshold is applied. A Canny [8] edge detector finds the lines in the contrast flow map. One line (or curve) in the contrast flow map indicates where the contrast agent enters parts of the vessel. The assumption that the contrast agent propagates with a constant velocity through the arteries allows us to use a line fit. The lines are found using the Hough transform [9]. All lines that do not have a negative slope are discarded as well as lines with a starting point after $1 / 2$ of the total aquisition time (we assume that the injection and full opacification of the coronary arteries happens in the first half of the recording). Evaluation of the algorithm is done by comparing the results of the TFC measurement algorithm with the TFC results from the expert.

\subsection{Experiments and results}

The method is evaluated on the coronary arteries of 22 patients from which 6 patients are imaged using a Philips Integris $\mathrm{H}(\mathrm{P})$ and 16 patients using a Siemens Axiom-Artis $(\mathrm{S}) \mathrm{C}$-arm. All the images are $[512 \times 512]$ pixels. The catheter location and the endpoint of one coronary artery are manually annotated throughout the whole image sequence by an expert. After this, the algorithm is applied. Figure 4.3 illustrates the results of our measurements. The ground truth (TFCM) was obtained by manual selection of the start and end frames by an expert. The automatic TFC (TFCA) is the output of our algorithm. The measurement is qualified as correct if the absolute difference between TFCA and TFCM is $\leq 5$, since 5 equals the intra observer difference $[10]$.

\subsection{Discussion}

As presented in table 4.3, the proposed method can estimate the contrast flow using TIMI frame counting in 13 of 22 cases within the expected range of $\mathrm{TFCM} \pm 5$. In figure $4.4,8$ flow maps within the error range are drawn. These 


\begin{tabular}{|c|c|c|c|}
\hline 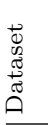 & 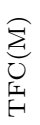 & 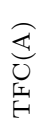 & 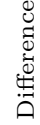 \\
\hline$\overline{1}$ & 21 & 15 & 6 \\
\hline 2 & 21 & 22 & 1 \\
\hline 3 & 18 & 23 & 5 \\
\hline 4 & 9 & 9 & 0 \\
\hline 5 & 16 & 17 & 1 \\
\hline 6 & 22 & 17 & 5 \\
\hline 7 & 22 & 25 & 3 \\
\hline $8^{a}$ & 26 & 17 & 9 \\
\hline 9 & 12 & 17 & 5 \\
\hline $10^{b}$ & 15 & 8 & 7 \\
\hline 11 & 24 & 26 & 2 \\
\hline 12 & 27 & 9 & 18 \\
\hline $13^{c}$ & 30 & 8 & 22 \\
\hline 14 & 25 & 7 & 18 \\
\hline 15 & 10 & 9 & 1 \\
\hline 16 & 10 & 9 & 1 \\
\hline $17^{d}$ & 31 & 7 & 24 \\
\hline 18 & 20 & 6 & 14 \\
\hline 19 & 16 & 6 & 10 \\
\hline 20 & 9 & 9 & 0 \\
\hline $21^{e}$ & 13 & 9 & 4 \\
\hline 22 & 11 & 9 & 2 \\
\hline
\end{tabular}

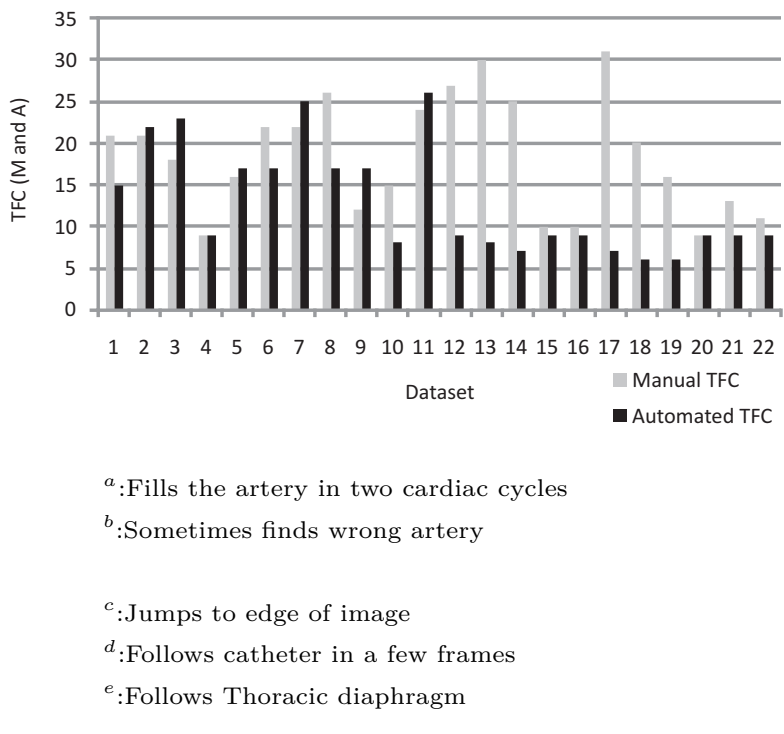

Figure 4.3: The results of the flow measurements using manual, $T F C(M)$, and automatic, $T F C(A)$, TIMI frame counting.

images clearly show an edge between the contrast and non-contrast regions, which is also detected by the algorithm. Figure 4.5 shows 6 flow maps of the data without the error range. There are a few reasons why the algorithm fails: Inaccurate vessel traces may occur when the selected artery is not completely in view throughout the sequence. In this case, it may happen that the boundary of the image is false identified as part of the artery. Another false tracking may occur when the catheter is clearly visible, the catheter may be tracked as part of the artery. The thoracic diaphragm can also be identified as a vessel. Since we assume that the contrast agent is linear propagated throughout the vessel, the algorithm fails when we should fit a curve in stead of a line. Most of the time this occurs when the distal part of the vessel is opacified one cardiac cycle later. This can also be seen in the flowmap from dataset 12. Furthermore, we have to take into account that we are dealing with a two-dimensional projection of a three dimensional tubular structure. Therefore, vessel structure perpendicular to the projection plane result in a higher opacity. This is clearly seen in the flow map of dataset 12 . 

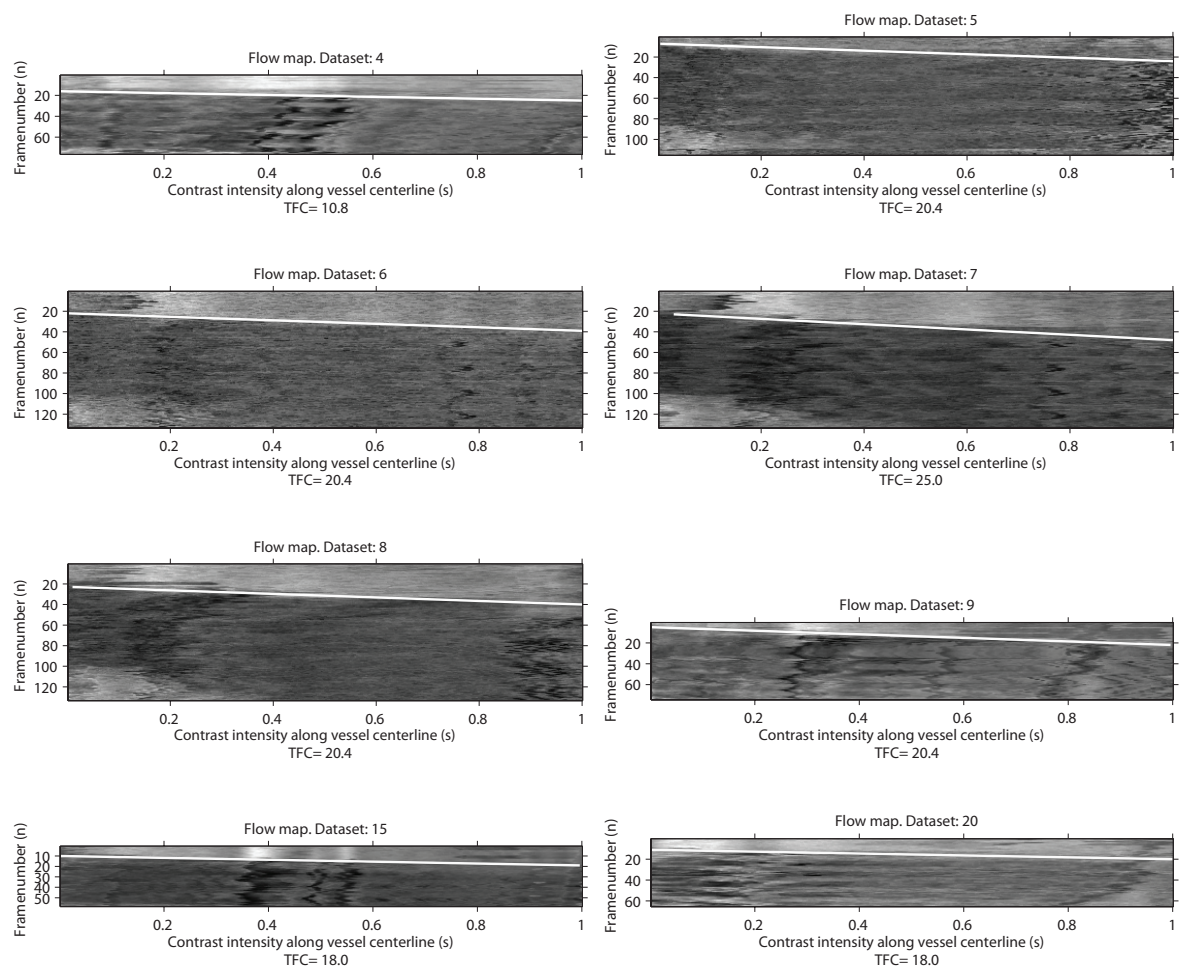

Figure 4.4: A set of flowmaps from automated TFC estimation within the error range \pm 5 .

\subsection{Conclusion}

The TIMI flow grading system is developed to give a semi-quantitative evaluation of the myocardial perfusion. Gibson extended this system by counting the number of frames between two defined points on a vessel. This system is called TIMI frame counting. We presented a method towards the automation of TIMI frame counting. Our algorithm follows the method used in clinical practice, the exception is that we not only measure the opacification of the vessel at the begin and endpoint of the vessel, but along the whole vessel centerline. As far as we know, the method presented is the only computer assisted method available for TIMI frame counting. It has the potential to reduce the intra observer variability and increase the reproducability of TIMI frame counting. Our method is able to give a TFC within the error range of \pm 5 frames from 13 out of 22 patients. Furthermore, quantification of the coronary flow can give use more specific information about the location of stenosis. To do this, we have to accurately find the vessels in the images. A 2-d projection of a three 

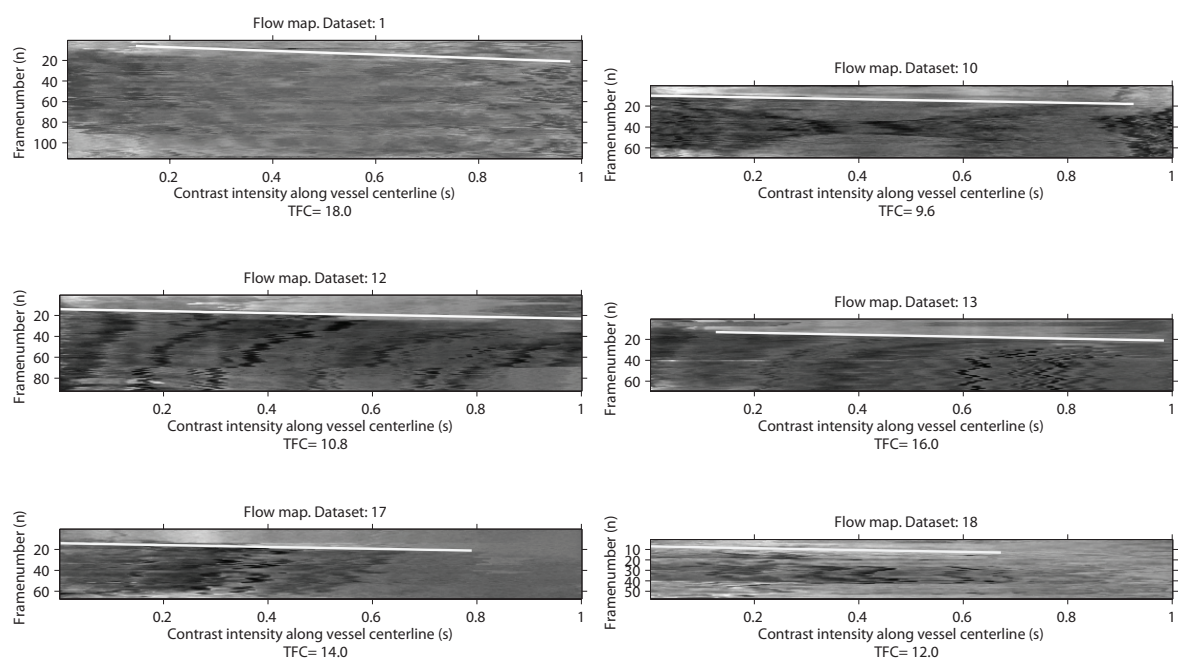

Figure 4.5: A set of flowmaps from automated TFC estimation outside the error range \pm 5 .

dimensional object like the coronaries of the heart, likely results in overlapping and foreshortening of vessels. 3-d model creation from these 2 -d images can help to separate these vessels.

\section{References}

[1] C. M. Gibson, C. P. Cannon, W. L. Daley, J. T. Dodge, B. Alexander, S. J. Marble, C. H. McCabe, L. Raymond, T. Fortin, and W. K. Poole, "TIMI frame count a quantitative method of assessing coronary artery flow," Circulation 93(5), p. 879-888, 1996.

[2] C. Bickel, H. J. Rupprecht, A. Maimaitiming, I. Welk, S. Blankenberg, F. Krummenauer, and J. Meyer, "The superiority of TIMI frame count in detecting coronary flow changes after coronary stenting compared to TIMI flow classification," The Journal of invasive cardiology 14(10), p. 590-596, 2002 .

[3] M. A. Appleby, A. D. Michaels, M. Chen, and C. G. Michael, "Importance of the TIMI frame count: implications for future trials," Current Controlled Trials in Cardiovascular Medicine 1(1), p. 31-34, 2000. PMC59594.

[4] M. G. Stoel, F. Zijlstra, and C. A. Visser, "Frame count reserve," Circulation 107, p. 3034-3039, June 2003. 
[5] N. E. Green, S. Y. J. Chen, J. C. Messenger, B. M. Groves, and J. D. Carroll, "Three-dimensional vascular angiography," Current Problems in Cardiology 29, p. 104-142, Mar. 2004.

[6] P. Fallavollita and F. Cheriet, "Robust coronary artery tracking from fluoroscopic image sequences," Lecture Notes in Computer Science 4633, p. $889,2007$.

[7] J. A. Sethian, "Evolution, implementation, and application of level set and fast marching methods for advancing fronts," Journal of Computational Physics 169(2), p. 503-555, 2001.

[8] J. Canny, "A computational approach to edge detection," IEEE Transactions on Pattern Analysis and Machine Intelligence 8, p. 679-698, 1986.

[9] J. Illingworth and J. Kittler, "A survey of the hough transform," Computer Vision, Graphics, and Image Processing 44(1), p. 87-116, 1988.

[10] J. K. French, C. J. Ellis, B. J. Webber, B. F. Williams, D. J. Amos, K. Ramanathan, R. M. L. Whitlock, and H. D. White, "Abnormal coronary flow in infarct arteries 1 year after myocardial infarction is predicted at 4 weeks by corrected thrombolysis in myocardial infarction (TIMI) frame count and stenosis severity," The American Journal of Cardiology 81, p. 665-671, Mar. 1998. 


\section{CHAPTER 5}

\section{Computer vision and coronary reconstruction}

Three dimensional modeling of the coronary arteries requires information about the geometry of the diagnostic $x$-ray imaging systems. In this chapter we will use uncalibrated mono-plane $x$-ray imaging to model the coronary arteries. First, the fundamental computer vision techniques such as coordinate systems, camera matrices, fundamental matrices and epipolar lines are discussed and applied to describe the C-arm geometry. Then, the three dimensional vessel reconstruction algorithm of Fallovalita et al. is extended with the usage of a Fast Marching Method based vessel tracing algorithm and applied to a set of clinical images. Our algorithm is validated using length measurements of the Left Anterior Descending (LAD) coronary artery. The length of the length of the LAD is measured in the three dimensional model and compared with in vivo catheter measurements from 30 patients. Experiments show an average error smaller than $20 \%$ of the LAD length.

\section{$5.1 \quad$ Introduction}

Coronary artery disease, or more specific a stenosis, may lead to a reduction in coronary blood flow. This is manifested in a reduced flow velocity of blood through the coronary arteries. Coronary flow velocity reserve is an important measure for heart assessment $[1,2,3]$. In clinical practice the method of TIMI frame counting can be considered as a qualitative flow velocity assessment using 2-d monoplane x-ray images [4]. This method, however, is manually performed by a cardiologist and requires catheter measurements to provide information about vessel length. In our research we are interested in the anatomy of the 
coronary arteries, and more specific the length of the vessels, because we want to estimate mean flow velocity. The standard minimally invasive modality to assess coronary arteries is mono-plane x-ray angiography, which is a two dimensional method. Three dimensional and also non-invasive methods are Computed Tomography (CT) and Magnetic Resonance Imaging (MRI). Several 3-d semi-automatic modeling methods have been proposed using mono-plane [5] and bi-plane x-ray [6]. 4-d models with motion analysis are shown by Chen et al. [7] and [8]. Tomographic reconstruction techniques require multiple projection angles which are obtained in $\mathrm{CT}$ or rotational angiography. This requires measuring of the electrocardiogram to perform ECG-gated recording or retrospective ECG gated reconstruction. Tomographic reconstruction requires a calibrated system in which the projection geometry is well defined and at least three projections are required to get reasonable results [9]. Several improvements of this algorithm are proposed by $[10,11,12]$. Coronary models can be used, for example, in intervention planning [13] or fusion with other modalities like IVUS [14]. In this chapter we assess the measurement of the LAD length using in vivo measurements of 30 patients and 3-d coronary modelling based on the algorithm of Fallavolita et al.[5].

\subsection{Projective geometry}

This section describes the geometry of the C-arm applied to the acquisition of the data used in this project. First, the coordinate system is defined, then a brief review on fundamental matrices and epipolar geometry is given. We construct a camera model from the information present in the Digital Imaging and Communications in Medicine (DICOM) file headers of the x-ray data.

Our camera model is defined in the coordinate system as shown in figure 5.1. The left image shows the 3 - $d$ and the right image the 2 -d version. The focal point is $c$ and the principle axis is the $z$ axis. The image plane is positioned at $f$, which is the distance to from the source $c$ to the detector (image plane). The imaged object is in between the image plane and the focal point, in contrast to optical camera models in which the image object is outside the projection plane and focal center. The right image shows the size of the detector (intensifier or image plane); $s_{i}$ is the size of the detector in $[\mathrm{mm}]$ and $d$ is half the size of the detector in [pixels].

Throughout this thesis we will use the pinhole camera model.In this model, see figure 5.1 reproduced from [15], the camera performs a perspective projection of an object point $p$ onto a pixel $p^{\prime}$ in the image plane through the optical center $c$. The optical axis is the z-axis. We define a three-dimensional orthonormal system of coordinates centered at the optical center $c$ with two axes of coordinates parallel to the image plane and the third one parallel to the optical axis. In these two systems of coordinates, the relationship between 


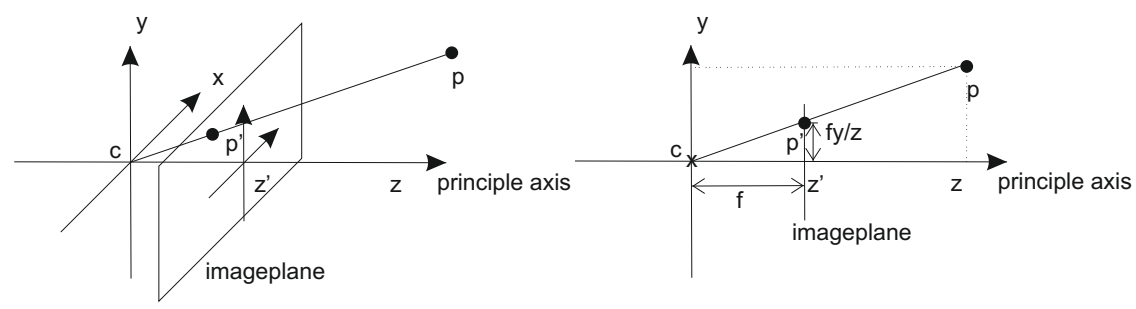

Figure 5.1: Pinhole camera model

the coordinates of $p^{\prime}$ and image of $p$ is [16]:

$$
p_{x}^{\prime}=\frac{p_{x}}{p_{z}} \quad p_{y}^{\prime}=\frac{p_{y}}{p_{z}}
$$

We linearize this equation by using the homogeneous projective coordinates of $p$ and $p^{\prime}[16]$ :

$$
\left[\begin{array}{l}
p_{x}^{\prime} \\
p_{y}^{\prime} \\
p_{w}^{\prime}
\end{array}\right]=\left[\begin{array}{llll}
1 & 0 & 0 & 0 \\
0 & 1 & 0 & 0 \\
0 & 0 & 1 & 0
\end{array}\right]\left[\begin{array}{c}
p_{x} \\
p_{y} \\
p_{z} \\
p_{w}
\end{array}\right]
$$

In this equation $p_{x}^{\prime}, p_{y}^{\prime}$ and $p_{w}^{\prime}$ are the homogeneous coordinates of the pixel $p^{\prime}$ and $p_{x}, p_{y}, p_{z}$ and $p_{w}$ are the homogeneous coordinates of the point $p$. If $p$ is not at infinity (i.e. $p_{w}^{\prime} \neq 0$ ), its Euclidean coordinates become $x=\frac{p_{x}^{\prime}}{p_{w}^{\prime}}, y=\frac{p_{y}^{\prime}}{p_{w}^{\prime}}$. In this way, we can do calculation mostly by additions and multiplications. In matrix form equation 5.2 is:

$$
\mathbf{p}_{e}=\mathbf{P}_{I} \mathbf{p}_{h}
$$

where $\mathbf{P}_{I}$ is the $[3 \times 4]$ matrix which transforms homogeneous coordinates $\mathbf{p}_{h}$ to Euclidean coordinates $\mathbf{p}_{e}$. We can add the matrix $\mathbf{D}$ (of rigid displacement):

$$
\mathbf{p}_{e}=\mathbf{P}_{I} \mathbf{D} \mathbf{p}_{h}
$$

in which:

$$
\mathbf{D}=\left[\begin{array}{cc}
\mathbf{R} & \mathbf{t} \\
0 & 1
\end{array}\right]
$$

The matrix $\mathbf{R}$ can be a $[3 \times 3]$ Euler rotation matrix $\mathbf{R}(\alpha)$, which rotates about an axis of the orthonormal coordinate system, or an axis angle rotation $\mathbf{Q}(\mathbf{v}, \alpha)$ which rotates around the axis $\mathbf{v}$ with angle $\alpha$ using quaternions. Vector $\mathbf{t}$ adds a translational component.

Euler rotations can be described using three equations, one for each rotation about axis $\mathrm{x}, \mathrm{y}$ or $\mathrm{z}$ :

$$
\mathbf{R}_{\mathbf{x}}(\alpha)=\left[\begin{array}{ccc}
1 & 0 & 0 \\
0 & \cos (\alpha) & -\sin (\alpha) \\
0 & \sin (\alpha) & \cos (\alpha)
\end{array}\right]
$$




$$
\begin{aligned}
& \mathbf{R}_{\mathbf{y}}(\alpha)=\left[\begin{array}{ccc}
\cos (\alpha) & 0 & \sin (\alpha) \\
0 & 1 & 0 \\
-\sin (\alpha) & 0 & \cos (\alpha)
\end{array}\right] \\
& \mathbf{R}_{\mathbf{z}}(\alpha)=\left[\begin{array}{ccc}
\cos (\alpha) & -\sin (\alpha) & 0 \\
\sin (\alpha) & \cos (\alpha) & 0 \\
0 & 0 & 1
\end{array}\right]
\end{aligned}
$$

A quaternion is the extension of the complex number. As we can perform 2 -d rotation in the complex plane by multiplication with $\mathbf{i}$, we can do the same in 4-d space using quaternions. In this case we have not only $\mathbf{i}$, but also $\mathbf{j}$ and k with similar properties [17]:

$$
\begin{array}{ll}
\mathbf{i} \cdot \mathbf{i} & =-1 \\
\mathbf{j} \cdot \mathbf{j} & =-1 \\
\mathbf{k} \cdot \mathbf{k} & =-1 \\
\mathbf{i} \cdot \mathbf{j} & =-\mathbf{j} \cdot \mathbf{i}=\mathbf{k} \\
\mathbf{j} \cdot \mathbf{k} & =-\mathbf{k} \cdot \mathbf{j}=\mathbf{i} \\
\mathbf{k} \cdot \mathbf{i} & =-\mathbf{i} \cdot \mathbf{k}=\mathbf{j}
\end{array}
$$

So we can represent a quaternion in the complex notation as $\mathbf{q}$ :

$$
\begin{aligned}
\mathbf{q}_{1} & =w_{1}+x_{1} \mathbf{i}+y_{1} \mathbf{j}+z_{1} \mathbf{k} \\
\mathbf{q}_{2} & w_{2}+x_{2} \mathbf{i}+y_{2} \mathbf{j}+z_{2} \mathbf{k} \\
\mathbf{q}_{1} \times \mathbf{q}_{2}= & {\left[\begin{array}{l}
w_{1} x_{2}+x_{1} w_{2}+y_{1} z_{2}-z_{1} y_{2} \\
w_{1} y_{2}-x_{1} z_{2}+y_{1} w_{2}+z_{1} x_{2} \\
w_{1} z_{2}+x_{1} y_{2}-y_{1} x_{2}+z_{1} w_{2} \\
w_{1} w_{2}-x_{1} x_{2}-y_{1} y_{2}-z_{1} z_{2}
\end{array}\right] \cdot\left[\begin{array}{c}
\mathbf{i} \\
\mathbf{j} \\
\mathbf{k} \\
1
\end{array}\right] }
\end{aligned}
$$

Based on row vectors for $\mathbf{q}_{1}$ and $\mathbf{q}_{2}$, we can rewrite the last equation in matrix form in two forms $\mathbf{M}_{\mathbf{l}}\left(\mathbf{q}_{\mathbf{1}}\right)$, see equation 5.11, and $\mathbf{M}_{\mathbf{r}}\left(\mathbf{q}_{2}\right)$, see equation 5.12:

$$
\begin{aligned}
& \mathbf{q}_{1} \times \mathbf{q}_{2}=\mathbf{q}_{2} \mathbf{M}_{l}\left(\mathbf{q}_{1}\right) \\
& =\left[\begin{array}{llll}
x_{2} & y_{2} & z_{2} & w_{2}
\end{array}\right]\left[\begin{array}{cccc}
w_{1} & z_{1} & -y_{1} & -x_{1} \\
-z_{1} & w_{1} & x_{1} & -y_{1} \\
y_{1} & -x_{1} & w_{1} & -z_{1} \\
x_{1} & y_{1} & z_{1} & w_{1}
\end{array}\right] \\
& \mathbf{q}_{1} \times \mathbf{q}_{2}=\mathbf{q}_{1} \mathbf{M}_{r}\left(\mathbf{q}_{2}\right) \\
& =\left[\begin{array}{llll}
x_{1} & y_{1} & z_{1} & w_{1}
\end{array}\right]\left[\begin{array}{cccc}
w_{2} & -z_{2} & y_{2} & -x_{2} \\
z_{2} & w_{2} & -x_{2} & -y_{2} \\
-y_{2} & x_{2} & w_{2} & -z_{2} \\
x_{2} & y_{2} & z_{2} & w_{2}
\end{array}\right]
\end{aligned}
$$




$$
\mathbf{Q}(\mathbf{q})=\mathbf{Q}=\left[\begin{array}{cccc}
w^{2}-z^{2}-y^{2}+x^{2} & 2 w z+2 y x & -2 w y+2 z x & 0 \\
-2 w z+2 y x & w^{2}-x^{2}+y^{2}-z^{2} & 2 z y+2 w x & 0 \\
2 w y+2 z x & 2 z y-2 w x & w^{2}-x^{2}-y^{2}+z^{2} & 0 \\
0 & 0 & 0 & x^{2}+y^{2}+z^{2}+w^{2}
\end{array}\right]
$$

A quaternion $\mathbf{Q}$ can rotate a vector $\mathbf{v}$ using the product $\mathbf{Q} \mathbf{v} \mathbf{Q}^{\mathbf{- 1}}$. This is only valid when using unit quaternions. Writing $\mathbf{P}=\left[x_{p}, y_{p}, z_{p}, 0\right]$ we get:

$$
\begin{aligned}
& \mathbf{P M}_{\mathbf{l}}(\mathbf{q})= \\
& =\left[\begin{array}{llll}
x_{p} & y_{p} & z_{p} & 0
\end{array}\right]\left[\begin{array}{rrrr}
w_{q} & z_{q} & -y_{q} & -x_{q} \\
-z_{q} & w_{q} & x_{q} & -y_{q} \\
y_{q} & -x_{q} & w_{q} & -z_{q} \\
x_{q} & y_{q} & z_{q} & w_{q}
\end{array}\right]
\end{aligned}
$$

And the other way around, with:

$$
\begin{aligned}
& \mathbf{q}^{-\mathbf{1}}=\mathbf{q}^{\prime}=\left[-x_{q},-y_{q},-z_{q}, w_{q}\right] \\
& \mathbf{P M}_{\mathbf{r}}\left(\mathbf{q}^{-\mathbf{1}}\right)= \\
& =\left[\begin{array}{llll}
x_{p} & y_{p} & z_{p} & 0
\end{array}\right]\left[\begin{array}{rrrr}
w_{q} & z_{q} & -y_{q} & x_{q} \\
-z_{q} & w_{q} & x_{q} & y_{q} \\
y_{q} & -x_{q} & w_{q} & z_{q} \\
-x_{q} & -y_{q} & -z_{q} & w_{q}
\end{array}\right] \\
& \mathbf{q} \mathbf{P q} \mathbf{q}^{\prime}=\mathbf{q}\left(\mathbf{P q}^{\prime}\right) \\
& =\mathbf{q}\left(\mathbf{P M}_{\mathbf{r}}\left(\mathbf{q}^{\prime}\right)\right) \\
& =\left(\mathbf{P} \mathbf{M}_{\mathbf{r}}\left(\mathbf{q}^{\prime}\right)\right) \mathbf{M}_{\mathbf{l}}(\mathbf{q}) \\
& =\mathbf{P}\left(\mathbf{M}_{\mathbf{r}}\left(\mathbf{q}^{\prime}\right) \mathbf{M}_{\mathbf{l}}(\mathbf{q})\right) \\
& =\mathbf{P Q}(\mathbf{q}) \\
& \mathbf{Q}(\mathbf{q})=\mathbf{M}_{\mathbf{r}}\left(\mathbf{q}^{-\mathbf{1}}\right) \mathbf{M}_{\mathbf{l}}(\mathbf{q})
\end{aligned}
$$

Finally, we can expand the definition of a quaternion to equation 5.18.

Calculating a 3 -d rotated point $\mathbf{P}_{\text {rot }}$ using quaternions is done by:

$$
\mathbf{P}_{\text {rot }}=\mathbf{P Q}
$$

We can use quaternions to rotate about an axis described by the (normalized) vector $\mathbf{v}=\left[v_{x}, v_{y}, v_{z}\right]$ and an angle $\alpha$ by using the following relations:

$$
\begin{aligned}
x & =v_{x} \sin (\alpha / 2) \\
y & =v_{y} \sin (\alpha / 2) \\
z & =v_{z} \sin (\alpha / 2) \\
w & =\cos (\alpha / 2)
\end{aligned}
$$


Cascading Euler rotations may result in rotation axis alignment. When this happens, rotations are not effective anymore. This effect is called Gimbal lock. Quaternions do not suffer from this effect and are easier to use in minimization routines due to their linear interpolation properties.

\subsection{Camera matrix from C-arm geometry}

A pinhole camera can be defined with the following equation [17]:

$$
\begin{gathered}
{\left[\begin{array}{c}
x \\
y \\
z \\
1
\end{array}\right] \mapsto\left[\begin{array}{c}
f x+z d_{x} \\
f y+z d_{y} \\
z
\end{array}\right]=\left[\begin{array}{cccc}
f & 0 & d_{x} & 0 \\
0 & f & d_{y} & 0 \\
0 & 0 & 1 & 0
\end{array}\right]\left[\begin{array}{l}
x \\
y \\
z \\
1
\end{array}\right]} \\
\mathbf{K}=\left[\begin{array}{ccc}
f & 0 & d_{x} \\
0 & f & d_{y} \\
0 & 0 & 1
\end{array}\right]
\end{gathered}
$$

The matrix $\mathbf{K}$ is called the camera calibration matrix. The vector $\mathbf{d}=$ $\left(d_{x}, d_{y}\right)$ is the displacement vector in the image plane, usually used to align the image plane coordinate system. Parameter $f$ is the focal distance. We can use this camera model by using:

$$
\mathbf{x}=\mathbf{K}\left[\begin{array}{llll}
1 & 0 & 0 & 0 \\
0 & 1 & 0 & 0 \\
0 & 0 & 1 & 0
\end{array}\right]\left[\begin{array}{l}
x \\
y \\
z \\
1
\end{array}\right]
$$

To map a point $\mathbf{X}_{\mathbf{h}}=(\mathrm{x}, \mathrm{y}, \mathrm{z}, \mathrm{w})$ in homogeneous coordinates back to a 3 -d point $\mathbf{X}$ in euclidean coordinates, we divide all components by the last component:

$$
\left[\begin{array}{l}
x \\
y \\
z \\
w
\end{array}\right] \mapsto\left[\begin{array}{l}
x / w \\
y / w \\
z / w
\end{array}\right]
$$

The information stored in the DICOM header is used to reconstruct the geometry of a C-arm. In our case the known variables are the width of the image plane $N$ in [pixels] and the width of the intensifier $D$ in $[\mathrm{mm}]$. We assume that the focal distance $f$ in $[\mathrm{mm}]$ is equal to the distance source to detector. Subsequently, we can calculate the pixel size $\mu=D / N$. We also introduce a displacement vector in the image plane $\mathbf{d}=\left[d_{x}, d_{y}\right]$. Variables $f, N$ and $D$ can be extracted from the DICOM info structure from fields Distance Source to Detector, Rows and Intensifier Size respectively. From these variables, we can setup the camera calibration matrix $\mathbf{K}$ : 


$$
\mathbf{K}=\left[\begin{array}{ccc}
f \mu & 0 & \frac{1}{2} N+d_{x} \\
0 & f \mu & \frac{1}{2} N+d_{y} \\
0 & 0 & 1
\end{array}\right]
$$

In figure 5.2 the coordinate system of the C-arm is drawn.

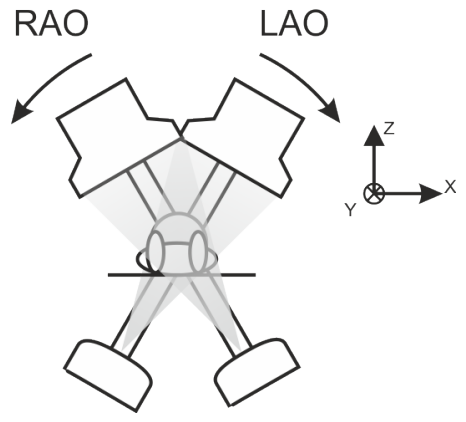

a: Primary Angle $(\alpha)$

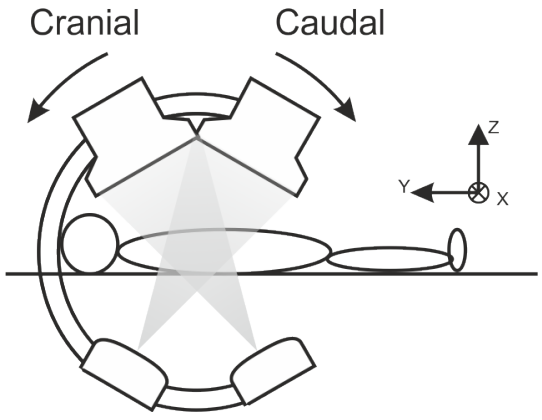

b: Secondary Angle $(\beta)$

Figure 5.2: The C-arm can rotate about two angles, the primary angle $\alpha$ (RAO/LAO), see fig $5.2 \mathrm{a}$, and the secondary angle $\beta$ (Caudal/Cranial), see fig $5.2 \mathrm{~b}$. The origin of the coordinate system is placed at the center of rotation of the C-arm. The $\mathrm{C}$-arm is at anterior-posterior $(\mathrm{PA})$ position when $[\alpha, \beta]=[0,0]$.

The rotation matrix $\mathbf{R}$ can be formed using the primary and secondary angle information from the DICOM info structure. The primary angle corresponds to RAO $(\alpha \leq 0)$ and LAO $(\alpha>0)$ projection and the secondary angle to the CAUD $(\beta \leq 0)$ and CRAN $(\beta>0)$ projection. The translation vector $\mathbf{t}$ is the position of the camera relative to the center of rotation.

$$
\begin{gathered}
\mathbf{R}_{\mathbf{x}}(\beta)=\left[\begin{array}{ccc}
1 & 0 & 0 \\
0 & \cos (\beta) & -\sin (\beta) \\
0 & \sin (\beta) & \cos (\beta)
\end{array}\right] \\
\mathbf{R}_{\mathbf{y}}\left(\alpha_{\mathbf{p}}\right)=\left[\begin{array}{ccc}
\cos (\alpha) & 0 & \sin (\alpha) \\
0 & 1 & 0 \\
-\sin (\alpha) & 0 & \cos (\alpha)
\end{array}\right] \\
\mathbf{R}(\alpha, \beta)=\mathbf{R}_{\mathbf{x}}(\beta) \mathbf{R}_{\mathbf{y}}(\alpha)
\end{gathered}
$$

The z-axis points towards the ceiling, so the camera is $f / 2$ out of the center of rotation in $z$ direction:

$$
\mathbf{t}=\left(\begin{array}{c}
0 \\
0 \\
f / 2
\end{array}\right)
$$


The camera matrix $\mathbf{P}$ is:

$$
\mathbf{P}=\mathbf{K}[\mathbf{R t}]
$$

\subsection{Essential and fundamental matrices}

The essential and fundamental matrices completely describe the geometric relationship between corresponding points of a pair of cameras. The only difference between the two is that the fundamental matrix deals with calibrated cameras and the essential matrix with uncalibrated cameras. The essential matrix contains three parameters for rotation and two for direction of translation. The magnitude of translation cannot be recovered due to scale invariance. Two important properties of the essential matrix are the zero determinant and the equality of its two non-zero singular values. The fundamental matrix contains two extra parameters for the calibration. It maps a point from the first projection plane $\mathbb{P}_{1}^{2}$ to the second projection plane $\mathbb{P}_{2}^{2}$, both defined in $\mathbb{R}^{2}$ so its rank is always two [16].

The fundamental matrix is a generalization of the essential matrix and contains no metric information. If a point $\mathbf{X}$ in $\mathbb{R}^{3}$ is imaged as $\mathbf{x}$ in the first view $\mathbb{P}_{1}^{2}$, and $\mathbf{x}^{\prime}$ in the second $\mathbb{P}_{2}^{2}$, then the image points satisfy the relation $\mathbf{x}^{\prime \top} \mathbf{F} \mathbf{x}=0$ [15]. The fundamental and essential matrix can be derived by starting with a stereo pair of cameras viewing a point $\mathbf{X}$ in the world which projects onto the two image planes at points $\mathbf{x}$ and $\mathbf{x}^{\prime}$. All points are expressed using homogenous coordinates, $\mathbf{X}$ is $[4 \times 1]$, and $\mathbf{x}$ and $\mathbf{x}^{\prime}$ are both $[3 \times 1]$. If we assume the cameras are calibrated, then $\mathbf{x}$ and $\mathbf{x}^{\prime}$ are given in normalized coordinates, that is, each is given with respect to its camera coordinate frame. The epipolar constraint dictates that the vector from the first camera optical center to the first imaged point, the vector from the second optical center to the second imaged point, and the vector from one optical center to the other are all co-planar. In normalized coordinates, this constraint can be expressed as

$$
\overline{\mathbf{x}}^{\prime \top}\left([\mathbf{t}]_{\times} \mathbf{R} \overline{\mathbf{x}}\right)=0
$$

where $\mathbf{R}$ and $\mathbf{t}$ describe the rotation and translation between the coordinate frames of the two cameras. The multiplication by $\mathbf{R}$ is necessary to transform $\overline{\mathbf{x}}$ into the second camera coordinate frame. $[\mathbf{t}]_{\times}$is defined as the matrix such that $[\mathbf{t}]_{\times} \mathbf{y}=\mathbf{t} \times \mathbf{y}$ for any vector $\mathbf{y}$. In this equation we recognize $\mathbf{E}=$ $[\mathbf{t}]_{\times} \mathbf{R}$ as the essential matrix. The essential matrix was introduced by LonguetHiggins(1981)[16] and covers the uncalibrated camera case. For the calibrated camera case, we introduce calibration matrices $\mathbf{K}_{\mathbf{1}}$ and $\mathbf{K}_{\mathbf{2}}$ containing the internal parameters of the two cameras. These are needed to transform the 
normalized coordinates into projection coordinates:

$$
\begin{aligned}
\mathbf{x} & =\mathbf{K} \overline{\mathbf{x}} \\
\mathbf{x}^{\prime} & =\mathbf{K}^{\prime} \overline{\mathbf{x}}^{\prime}
\end{aligned}
$$

This yields the following equation 5.32, where $\mathbf{F}=\mathbf{K}^{\prime-\top} \mathbf{E} \mathbf{K}^{-\mathbf{1}}$ is the fundamental matrix.

$$
\begin{aligned}
\left(\mathbf{K}^{\prime-1} \mathbf{x}^{\prime}\right)^{\top}\left([\mathbf{t}]_{\times} \mathbf{R} \mathbf{K}^{-1} \mathbf{x}\right) & = \\
\mathbf{x}^{\prime \top} \mathbf{K}^{\prime-\top}\left([\mathbf{t}]_{\times} \mathbf{R} \mathbf{K}^{-1} \mathbf{x}\right) & = \\
\mathbf{x}^{\prime \top} \mathbf{F} \mathbf{x} & =0
\end{aligned}
$$

The fundamental matrix can be calculated from seven points, two planes, or by using the so called Gold Standard Algorithm [15]. In this section we only describe the Gold Standard algorithm, since we are also using a similar approach to calculate displacement of the image plane due to patient/table movement.

The Gold Standard algorithm depends on an error criterium based on the reprojection error. The errorfunction can be described as:

$$
\sum_{i}\left\|x_{i}, \hat{x}_{i}\right\|^{2}+\left\|x_{i}^{\prime}, \hat{x}_{i}^{\prime}\right\|^{2}
$$

In our case we propose to calculate the fundamental matrix using two cameras. Then we calculate the 3 -d points using triangulation. After triangulation, we reproject. The method used in this thesis to calculated the fundamental matrix requires two camera matrices $\mathbf{A}$ and $\mathbf{B}$. The relation world to image coordinates can be expressed as $\kappa \mathbf{x}=A \mathbf{X}$ and $\kappa^{\prime} \mathbf{x}^{\prime}=B \mathbf{X}$ where $\kappa$ and $\kappa^{\prime}$ are constants. The two equations can be written as:

$$
\left[\begin{array}{ccc}
\mathbf{A} & \mathbf{x} & \mathbf{0} \\
\mathbf{B} & \mathbf{0} & \mathbf{x}^{\prime}
\end{array}\right]\left(\begin{array}{c}
\mathbf{X} \\
-\kappa \\
-\kappa^{\prime}
\end{array}\right)=\mathbf{0}
$$

This can be rewritten by using the notation $\mathbf{a}^{i}$ for the $i$-th row of matrix $A$ and similarly for matrix $B$. Also, using the same notation, $\mathbf{x}$ and $\mathbf{x}^{\prime}$ can be rewritten as $\left(x^{1}, x^{2}, x^{3}\right)^{\top}$ and $\left(x^{\prime 1}, x^{\prime 2}, x^{\prime 3}\right)^{\top}$, respectively. The equation becomes:

$$
\left[\begin{array}{ccc}
\mathbf{a}^{1} & x^{1} & \\
\mathbf{a}^{2} & x^{2} & \\
\mathbf{a}^{3} & x^{3} & \\
\hline \mathbf{b}^{1} & & x^{\prime 1} \\
\mathbf{b}^{2} & & x^{\prime 2} \\
\mathbf{b}^{3} & & x^{\prime 3}
\end{array}\right]\left(\begin{array}{c}
\mathbf{X} \\
-\kappa \\
-\kappa^{\prime}
\end{array}\right)=\mathbf{0}
$$


We assume that the vector $\left(\mathbf{X}^{\top},-\kappa,-\kappa^{\prime}\right)^{\top}$ is a non-zero solution of the set of equations described by the matrix in equation 5.35. This requires that the determinant of the matrix must be zero. The relation between $\mathbf{x}$ and $\mathbf{x}^{\prime}$ can be described by the fundamental matrix $\mathbf{F}$ as:

$$
\mathbf{x}^{\prime} \mathbf{F} \mathbf{x}^{\top}=\left(x^{\prime 1}, x^{\prime 2}, x^{\prime 3}\right) F\left(x^{1}, x^{2}, x^{3}\right)^{\top}=x^{\prime i} x^{j} F_{i j}=0
$$

The value $F_{i j}$ is the coefficient of the term $x^{i} x^{j}$ in the expansion of the determinant of the matrix in equation 5.35. Each coefficient can be found by calculating the determinant after elimination of the rows and columns containing terms $x^{i}$ and $x^{\prime} j$. When we write $\sim \mathbf{a}^{i}$ as the matrix obtained after eliminating the $i$ th row from matrix $\mathbf{A}$, then we can write:

$$
F_{j i}=(-1)^{i+j} \operatorname{det}\left[\begin{array}{l}
\sim \mathbf{a}^{i} \\
\sim \mathbf{b}^{j}
\end{array}\right]
$$

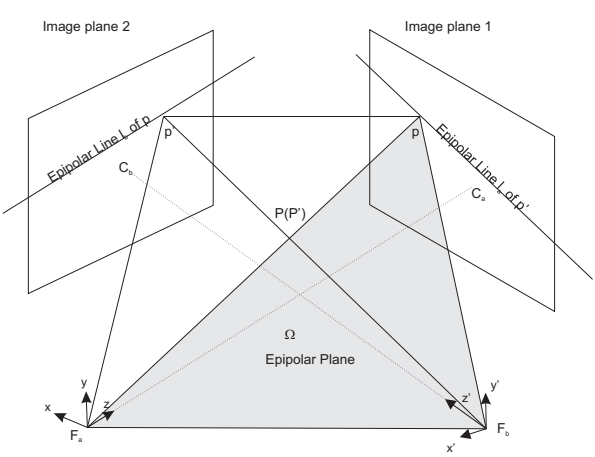

Figure 5.3: Epipolar line, image reproduced from [7]

The epipolar geometry is the intrinsic projective geometry between two views. It is independent of scene structure and only depends on the internal parameters and relative pose of the cameras. The fundamental matrix $\mathbf{F}$ encapsulates this intrinsic geometry [15]. The epipolar geometry is the basic constraint which arises from the existence of two viewpoints [16]. This constraint allows us to calculate the projection of a point from the first view onto the second view. If the cameras do not share the same center and are not parallel, then we can write down the equation of the epipolar lines:

$$
\mathbf{l}_{2}^{\prime}=\mathbf{F} \mathbf{x}_{1} \quad \text { and } \quad \mathbf{l}_{1}=\mathbf{F}^{\top} \mathbf{x}_{2}^{\prime}
$$

For the projection of point $\mathbf{x}_{1}$ of the first plane into the line $\mathbf{l}_{2}^{\prime}$ in the second plane and viceversa. 


\subsection{Three dimensional reconstruction methods}

Several 3-d modeling and reconstruction methods can be found in literature $[18,8,19,5,20]$. In this section we review the basics of the algorithms from Chen et al. [18], Canero et al. [19] and Fallavollita et al. [5].

A reconstructon algorithm proposed by [18] uses bifurcation points and directional vectors. The spatial relationship between the two views can be characterized by:

$$
\begin{aligned}
{\left[\begin{array}{l}
x_{i}^{\prime} \\
y_{i}^{\prime} \\
z_{i}^{\prime}
\end{array}\right] } & \left.=\mathbf{R} \cdot\left\{\left[\begin{array}{l}
x_{i} \\
y_{i} \\
z_{i}
\end{array}\right]-\mathbf{t}\right\}\right\} \\
& =\left[\begin{array}{lll}
r_{11} & r_{12} & r_{13} \\
r_{21} & r_{22} & r_{23} \\
r_{31} & r_{32} & r_{33}
\end{array}\right] \cdot\left[\begin{array}{l}
x_{i}-t_{x} \\
y_{i}-t_{y} \\
z_{i}-t_{z}
\end{array}\right]
\end{aligned}
$$

Then, the following function is minimized:

$$
\begin{aligned}
\frac{\min }{p, p^{\prime}, v, v^{\prime}} \mathcal{F}( & \left.P, P^{\prime}, v, v^{\prime}\right)= \\
& \sum_{i=1}^{n}\left\{\left(\xi_{i}-\frac{x_{i}}{z_{i}}\right)^{2}+\left(\eta_{i}-\frac{y_{i}}{z_{i}}\right)^{2}+\right. \\
& \left(\xi_{i}^{\prime}-\frac{x_{i}^{\prime}}{z_{i}^{\prime}}\right)^{2}+\left(\eta_{i}^{\prime}-\frac{y_{i}^{\prime}}{z_{i}^{\prime}}\right)^{2}+ \\
& \left.\left\|\nu_{i}-\left[v_{i}\right]_{x y}\right\|^{2}+\left\|\nu_{i}^{\prime}-\left[v_{i}^{\prime}\right]_{x^{\prime} y^{\prime}}\right\|^{2}\right\}
\end{aligned}
$$

where $n$ denotes the number of pairs of corresponding points extracted from the two images. $P$ and $P^{\prime}$ represent the sets of 3 -d object position vectors $\mathbf{p}_{i}=\left(x_{i}, y_{i}, z_{i}\right)$ and $\mathbf{p}_{i}^{\prime}=\left(x_{i}^{\prime}, y_{i}^{\prime}, z_{i}^{\prime}\right)$ characterized by 5.39. $\nu_{i}$ and $\nu_{i}^{\prime}$ denote the respective 3 -d vessel directional vectors of bifurcations in each view.

$\mathbf{v}=\left\{v_{1}, v_{2}, \cdots, v_{n}\right\}$ and $\mathbf{v}^{\prime}=\left\{v_{1}^{\prime}, v_{2}^{\prime}, \cdots, v_{n}^{\prime}\right\}$ represent the calculated $3-\mathrm{d}$ vessel directional vectors of bifurcations defined in the respective coordinate systems of the two views.

$\left[v_{i}\right]_{x y}$ and $\left[v^{\prime}\right]_{x^{\prime} y^{\prime}}, i=1, \cdots, n$, represent the calculated 2 -d projection vectors from $v_{i}$ and $v_{i}^{\prime}$ onto the image planes respectively.

The relationship between the two views can be characterized by a rotation matrix $\mathbf{R}$ and a translation vector $\mathbf{t}=\left[t_{x}, t_{y}, t_{z}\right]$ as shown in equation 5.39 and 5.40 .

Fallavollita et al. [5] proposed a method to estimate coronary arteries using snakes. Our method uses this approach with the main difference that a FMM speed-map is used as force function. The force function is important because it controls the deformation of the 3 -d snake. The snake is described by a $3-\mathrm{d}-$ curve or B-spline $\nu(s)$. The deformation is controlled by an energy minimization function:

$$
E(\nu)=\int_{0}^{1}\left(E_{\text {int }}(\nu)+E_{\text {ext }}(\nu)\right) d s
$$


where $E_{\text {int }}(\nu)$ is the internal energy, preserving smoothness, and $E_{\text {ext }}(\nu)$ is the external energy, attracting the snake to image features. $E_{\text {int }}$ can be described by:

$$
E_{\text {int }}(\nu)=\gamma\left|\frac{\partial \nu}{\partial s}\right|^{2}+\lambda\left|\frac{\partial^{2} \nu}{\partial s^{2}}\right|^{2}
$$

where $\gamma$ and $\lambda$ are constants controlling the tension and rigidity of the snake respectively.

The external energy $E_{\text {ext }}$ can be described by:

$$
E_{\text {ext }}(\nu)=\phi^{-1}\left(\mathbf{x}_{1}, \mathbf{x}_{2}, \mathbf{P}_{1}, \mathbf{P}_{2}\right)-\mathbf{X}
$$

where $\phi^{-1}$ is the retro-projection operator. This operator reconstructs a 3 -d point from two given 2-d points $\mathbf{x}_{n}$ in two projection planes $n=1,2$ described by camera matrix $\mathbf{P}_{n}$ :

$$
\mathbf{X}=\phi^{-1}\left(\mathbf{x}_{1}, \mathbf{x}_{2}, \mathbf{P}_{1}, \mathbf{P}_{2}\right)
$$

$\mathbf{x}_{n}$ are the original points $\mathbf{q}_{n}$ with a movement depending on the force maps $\mathbf{F}_{n}$ :

$$
\mathbf{x}_{n}=\mathbf{q}_{n}-\nabla \mathbf{F}_{n}\left(\mathbf{q}_{n}\right)
$$

where $\mathbf{q}_{n}$ are the projections of $\nu$ on projection planes $n=1,2$ and $\mathbf{F}_{n}$ are the force maps, they are discussed in the next section.

The numerical implementation of the snake algorithm requires approximation of the derivatives with finite differences. Conversion to vector notation with $\nu_{i}=\left(x_{i}, y_{i}, z_{i}\right)[21]$ results in:

$$
\begin{aligned}
E_{i n t}(\nu)=\gamma \mid \nu_{i}- & \left.\nu_{i-1}\right|^{2}+\lambda\left|\nu_{i-1}-2 \nu_{i}+\nu_{i+1}\right|^{2} \\
\gamma\left|\frac{\partial \nu_{i}}{\partial s}\right|^{2} & \approx \gamma\left|\nu_{i}-\nu_{i-1}\right|^{2} \\
& =\gamma\left(\left(x_{i}-x_{i-1}\right)^{2}\right. \\
& +\left(y_{i}-y_{i-1}\right)^{2} \\
& \left.+\left(z_{i}-z_{i-1}\right)^{2}\right) \\
\lambda\left|\frac{\partial^{2} \nu_{i}}{\partial s^{2}}\right|^{2} & \lambda\left|\nu_{i-1}-2 \nu_{i}+\nu_{i+1}\right|^{2} \\
= & \lambda\left(\left(x_{i-1}-2 x_{i}+x_{i+1}\right)^{2}\right. \\
+ & \left(y_{i-1}-2 y_{i}+y_{i+1}\right)^{2} \\
+ & \left.\left(z_{i-1}-2 z_{i}+z_{i+1}\right)^{2}\right)
\end{aligned}
$$

The distance between the points is kept equidistant (Euclidean distance) by redistribution of the points along the snake using cubib B-spline interpolation. Discretization of the integral in equation 5.41 gives:

$$
E=\sum_{i=0}^{N-1}\left(E_{\text {int }}\left(\nu_{i}\right)+E_{\text {ext }}\left(\nu_{i}\right)\right)
$$


for a snake with $N$ nodes.

Minimization of $E$ allows us to rewrite equation 5.49 for solving using dynamic programming:

$$
\begin{aligned}
E\left(\nu_{1}, \nu_{2}, \ldots, \nu_{N}\right) & =E_{1}\left(\nu_{1}, \nu_{2}\right) \\
& +E_{2}\left(\nu_{2}, \nu_{3}\right)+\ldots \\
& +E_{N-1}\left(\nu_{N-1}, \nu_{N}\right)
\end{aligned}
$$

In case of, for example, $N=5$ nodes we can calculate the minimal energy using sub-functions $s_{k}\left(\nu_{k+1}\right)$ :

$$
\begin{aligned}
s_{1}\left(\nu_{2}\right) & =\min _{\nu_{1}} E_{1}\left(\nu_{1}, \nu_{2}\right) \\
s_{2}\left(\nu_{3}\right) & =\min _{\nu_{2}}\left(s_{1}\left(\nu_{2}\right)+E_{2}\left(\nu_{2}, \nu_{3}\right)\right) \\
s_{3}\left(\nu_{4}\right) & =\min _{\nu_{3}}\left(s_{2}\left(\nu_{3}\right)+E_{3}\left(\nu_{3}, \nu_{4}\right)\right) \\
\min _{\nu_{1}, \ldots, \nu_{5}} E\left(\nu_{1}, \ldots, \nu_{5}\right) & =\min _{\nu_{4}}\left(s_{3}\left(\nu_{4}\right)+E_{4}\left(\nu_{4}, \nu_{5}\right)\right)
\end{aligned}
$$

The recurrence relation is now stated as (for clarity the second order term is not presented):

$$
\begin{aligned}
& s_{k}\left(\nu_{k+1}\right)= \\
& \min _{\nu_{k}}\left\{s_{k-1}\left(\nu_{k}\right)+E_{\text {ext }}\left(\nu_{k}\right)+\left|\nu_{k+1}-\nu_{k}\right|^{2}\right\}
\end{aligned}
$$

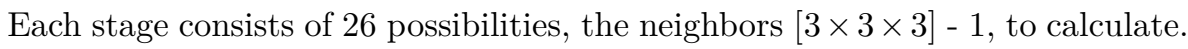
The internal energy is stored at each stage. The indices of node position with the minimum energy cost are stored in the position matrix. The minimum energy can now be found by back-tracing in the position matrix using Dijkstra's shortest path algoritm [22].

\subsubsection{Force map}

The force map is specifically constructed for each vessel segment to prevent interference from other vessel segments during the minimization process of the 3 -d-snake. The begin and endpoint of each vessel segment is annotated. With this annotation we can generate a force map from each vessel segment using the Fast Marching Method (FMM) [23]. This force map is used to find the vessel centerline using the minimal cost path algorithm. The 2 - $\mathrm{d}$ vesselcenterline is plotted in a 2-d grid. From this grid a euclidean distance map is calculated. The gradient of the distance map is used as force map in the snake energy minimization algorithm.

The vessel centerline is found using the 2-d multi-stencils fast marching method (FMM) [24]. This FMM is used to build a map containing the travel time between the start-point and all other points using a force function, which can be considered as the speed. The FMM solves the Eikonal equation:

$$
\|\nabla T(x, y)\| F(x, y)=1
$$



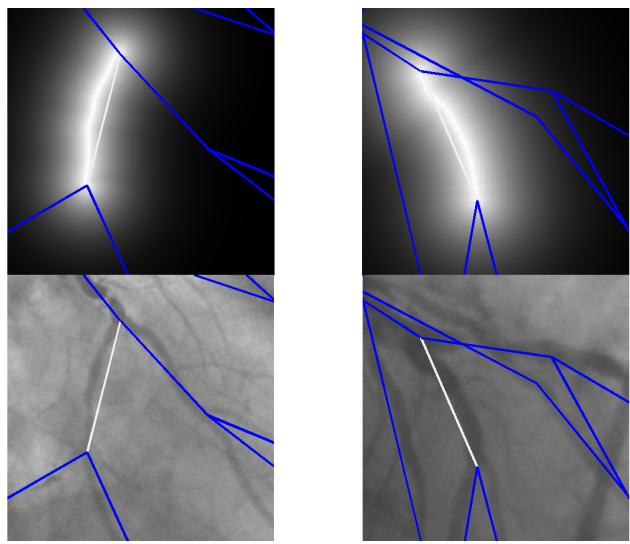

Figure 5.4: The bottom two images show the annotation of the vessel segments with a highlight (in white) of the vessel segment currently being traced. The upper figure shows the euclidean distance map after vessel detection using the FMM method and minimal cost path algorithm. The gradient of the distance map shown in the upper figures are used as force field in the snake deformation algorithm.

in which $T(x, y)$ is the arrival time of the front and $F(x, y)$ is the force function. The contrast enhanced image $I_{c}(x, y)$ is used as input $F(x, y)$ for the fast marching method:

$$
F(x, y)=\left(1-\left(G_{\sigma} \otimes I_{c}\right)\right)^{\gamma}
$$

Now the vessel centerline is the minimum cost path from the starting point to the end point, the back-tracing of the minimal cost past is performed using a fourth order Runge-Kutta approximation. The centerline is defined by $\nu(s)=$ $(x(s), y(s))$ where $x$ and $y$ are the coordinate functions and $s \in[0,1]$ is the parametric domain which describes the vessel from start to endpoint.

\subsubsection{Table motion compensation}

Table motion is compensated using image plane shifting. The only requirement for this algorithm is the annotation of the catheter-tip throughout the image sequence. The correction is performed in the $\mathrm{x}-\mathrm{y}$ plane of the local coordinate system, this results in a converging solution. Equation 5.30 is changed to include the table translation in the $\mathrm{x}-\mathrm{y}$ plane:

$$
\mathbf{P}=\mathbf{K}\left[\mathbf{R} \mid\left[\mathbf{t}+\mathbf{R}^{-1} \mathbf{t}_{t}\right]\right]
$$

In which $\mathbf{t}_{t}$ is the vector:

$$
\mathbf{t}_{t}=\left[\begin{array}{c}
x_{t} \\
y_{t} \\
0
\end{array}\right]
$$


This vector has two parameters $x_{t}$ and $y_{t}$. These parameters represent table motion, which results in an $\mathrm{x}-\mathrm{y}$ motion in the image plane. The parameters can be solved by minimizing the re-projection error of the catheter-tip in the second image plane using equation 5.43. The method solves the triangulation problem using the direct linear transform. This method is described in detail in Hartley and Zisserman [15].

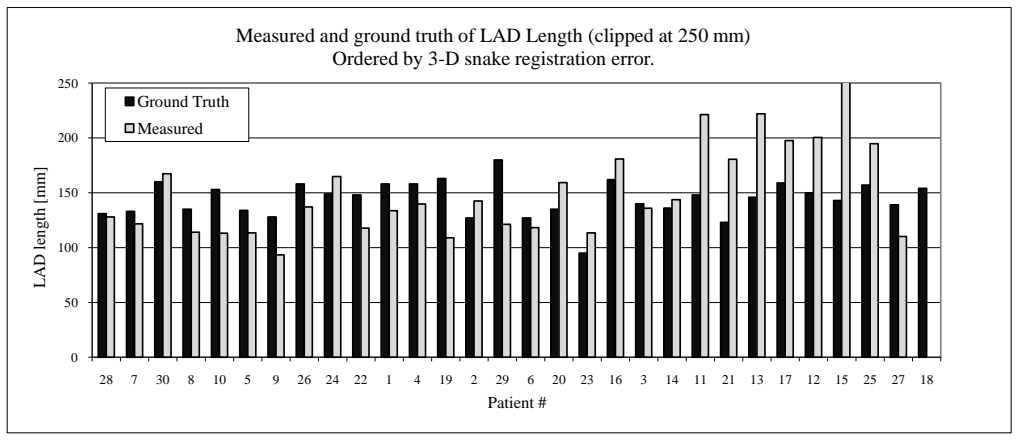

Figure 5.5: Length of the Left Anterior Descending (LAD) artery. The length calculated from the 3-d model is compared to the length measured with a catheter (ground thruth). The patients are ordered by registration energy $E$.

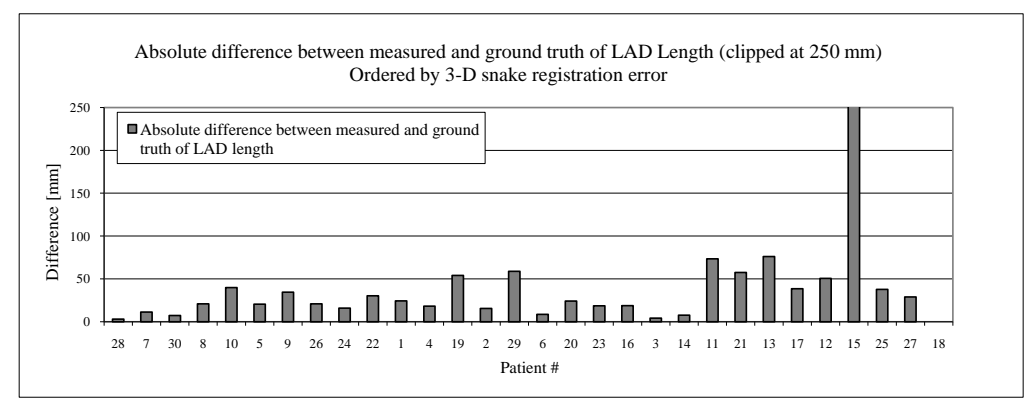

Figure 5.6: Length of the Left Anterior Descending (LAD) artery. The diffference between the length calculated from the 3 -d model and the length measured with a catheter (ground thruth) is shown in the graph. The patients are ordererd by registration energy $E$. In this figure we can clearly show that the registration energy $E$ can be used to reject $3-\mathrm{d}$ models.

\subsection{Experiments}

In our experiments we implemented the 3-d reconstruction method as described above and compared the length of the LAD measured from the reconstructed 


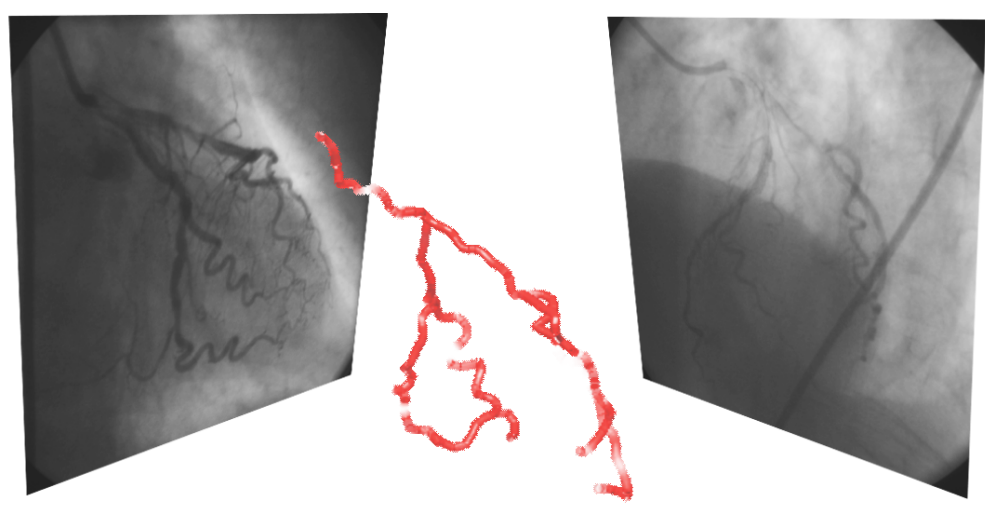

Figure 5.7: 4-d model $\mathcal{T}$ creation using mono-plane x-ray sequences at two different projection angles. The frames are selected using retrospective ECG gating. The 4-d cardiac cycle is covered by a set of 3 -d models $\mathcal{T}=\left[\mathcal{M}_{0}, \ldots, \mathcal{M}_{1}\right]$ estimated from the 2-d images $\mathcal{I}$.

coronary artery with the ground truth obtained by catheter measurements. The catheter measurements start from the left main artery to the last apical bifurcation of the LAD. In the measurements from the reconstructed coronary arteries we only measure from the start of the LAD to the last bifurcation of the LAD on the apex.

\subsection{Results}

Our dataset contains 30 patients (10 female, 20 male) from which a total of 13096 images have been semi automatically annotated. From all patients 20 models covering the cardiac cycle are reconstucted using the method described in this paper. From these models the mean artery lengths are calculated. We compare the calculated mean length of the LAD with the length of the LAD which is known from catheter measurements. The results are displayed in figure 5.5 and figure 5.6. The ordering of the patients is based on the registration energy $E$, see equation 5.49 , which can be considered as a quality measure for the reconstructed model. Figure 5.7 shows an example of a three dimensional model of the left coronary artery using only two images which are selected by retrospecive ECG gating.

\subsection{Discussion and conclusion}

In this thesis we present LAD length measurements using uncalibrated planar $\mathrm{x}$-ray imaging and 3-d modeling. The LAD length is compared to the 'ground 
thruth', which are the catheter measurements performed by a cardiologist [4]. Although we have used uncalibrated image sequences and rely only on the geometry provided by the DICOM data, we are able to estimate the LAD length with an average error compared to the catheter measured LAD that is smaller than $20 \%$. We have to note, however, that the measurements performed by the cardiologist include the left main artery. We only measured the LAD from the start until the last apical bifurcation.

\section{References}

[1] J. H. C. Reiber, P. W. Serruys, and C. J. Slager, Quantitative coronary and left ventricular cineangiography : methodology and clinical applications, Developments in cardiovascular medicine, Boston ; Dordrecht : Nijhoff, 1986.

[2] M. Schrijver, Angiographic Image Analysis to Assess the Severity of Coronary Stenosis. PhD thesis, University of Twente, 2002.

[3] N. P. Csizmadia, M. Schrijver, C. H. Slump, and A. P. G. Lubbers, "Digital densitometric determination of relative coronary flow distributions," Medical and Biological engineering and computing 39, p. 303-309, Feb. 2001.

[4] M. G. Stoel, F. Zijlstra, and C. A. Visser, "Frame count reserve," Circulation 107, p. 3034-3039, June 2003.

[5] P. Fallavollita and F. Cheriet, "Optimal 3D reconstruction of coronary arteries for 3D clinical assessment.," Computerized Medical Imaging and Graphics 32(6), pp. 476-487, 2008.

[6] S. Y. J. Chen and C. E. Metz, "Improved determination of biplane imaging geometry from two projection images and its application to threedimensional reconstruction of coronary arterial trees," Medical Physics 24, p. $633,1997$.

[7] S. Y. J. Chen and J. D. Carroll, "Kinematic and deformation analysis of 4-D coronary arterial trees reconstructed from cine angiograms," Medical Imaging, IEEE Transactions on 22(6), p. 710-721, 2003.

[8] C. Blondel, G. Malandain, R. Vaillant, and N. Ayache, "Reconstruction of coronary arteries from a single rotational x-ray projection sequence," IEEE Transactions on Medical Imaging 25(5), p. 653-663, 2006.

[9] R. Gordon, R. Bender, and G. T. Herman, "Algebraic reconstruction techniques (ART) for three-dimensional electron microscopy and x-ray photography," Journal of Theoretical Biology 29, p. 471-481, Dec. 1970. 
[10] M. Li, H. Yang, and H. Kudo, "An accurate iterative reconstruction algorithm for sparse objects: application to 3D blood vessel reconstruction from a limited number of projections," Physics in Medicine and Biology 47(15), p. 2599-2609, 2002.

[11] M. Li, H. Kudo, J. Hu, and R. H. Johnson, "Improved iterative algorithm for sparse object reconstruction and its performance evaluation with microCT data," Nuclear Science, IEEE Transactions on 51(3), p. 659-666, 2004 .

[12] E. Hansis, D. Schäfer, O. Dössel, and M. Grass, "Evaluation of iterative sparse object reconstruction from few projections for 3-D rotational coronary angiography.," IEEE transactions on medical imaging 27(11), p. $1548,2008$.

[13] O. Wink, R. Kemkers, S. Y. Chen, and J. D. Carroll, "Intra-procedural coronary intervention planning using hybrid 3-dimensional reconstruction techniques," Academic radiology 10(12), p. 1433-1441, 2003.

[14] K. R. Hoffmann, A. Wahle, C. Pellot-Barakat, J. Sklansky, and M. Sonka, "Biplane x-ray angiograms, intravascular ultrasound, and 3D visualization of coronary vessels," The International Journal of Cardiac Imaging 15, p. 495-512, Dec. 1999.

[15] R. Hartley and A. Zisserman, Multiple View Geometry in Computer Vision, Cambridge University Press, 2000.

[16] Q. T. Luong and O. D. Faugeras, "The fundamental matrix: Theory, algorithms, and stability analysis," International Journal of Computer Vision 17(1), p. $43-75,1996$.

[17] J. Arvo, Graphics Gems II, Academic Press, 1991.

[18] S. J. Chen and J. D. Carroll, "3-D reconstruction of coronary arterial tree to optimizeangiographic visualization," Medical Imaging, IEEE Transactions on 19(4), p. 318-336, 2000.

[19] C. Canero, P. Radeva, R. Toledo, J. Villanueva, and J. Mauri, "3D curve reconstruction by biplane snakes," in International Conference on Pattern Recognition, 15, p. 563-566, 2000.

[20] I. Waechter, J. Bredno, J. Weese, D. C. Barratt, and D. J. Hawkes, "Using flow information to support 3D vessel reconstruction from rotational angiography," Medical Physics 35, p. 3302-3316, July 2008.

[21] A. Amini, T. E. Weymouth, and R. C. Jain, "Using dynamic programming for solving variational problems in vision," IEEE transactions on pattern analysis and machine intelligence 12(9), p. 855-867, 1990. 
[22] E. W. Dijkstra, "A note on two problems in connexion with graphs," Numerische Mathematik 1, pp. 269-271, Dec. 1959.

[23] J. A. Sethian, "Evolution, implementation, and application of level set and fast marching methods for advancing fronts," Journal of Computational Physics 169(2), p. 503-555, 2001.

[24] M. S. Hassouna and A. A. Farag, "Multistencils fast marching methods: A highly accurate solution to the eikonal equation on cartesian domains," IEEE Transactions on Pattern Analysis and Machine Intelligence 29(9), p. 1563-1574, 2007. 


\section{CHAPTER 6}

\section{Coronary modelling in $3-\mathrm{d}^{1}$}

In coronary angiography the coronary blood flow velocity can be assessed by tracking a contrast agent in an image sequence. The contrast agent flow velocity is used to estimate the functional behavior of the coronary arteries using TIMI frame count (TFC). In this thesis we descibe the 3-d reconstruction algorithm used in our method towards the automation of TFC. The method creates a two dimensional map of the contrast agent in which the opacification of the vessel centerline is plotted against time. This map is used to find the velocity of the contrast agent and subsequently the TFC. The vessel centerline is obtained using the Fast Marching Method to find the minimum cost path between the catheter point and the end of the vessel. The determination of the start and endpoint is estimated using a $3-d$ model reconstructed from bi-plane $2-d$ image data. The final reconstructed coronary model only includes the segments matching our error criterion.

\subsection{Introduction}

In [2], we proposed the creation of a flowmap to measure coronary flow velocity. The flowmap is a two dimensional map of the contrast agent in which the opacification of the vessel centerline is plotted against time. This flowmap is used to find the velocity of the contrast agent and subsequently the TFC. An example flowmap is shown in figure 6.1. Currently, the vessel centerline is obtained using the Fast Marching Method to find the lowest cost path between the catheter point and the manually selected endpoint of the vessel. Fitting a

\footnotetext{
${ }^{1}$ This chapter is published at the IEEE EMBS 2009. [1]
} 


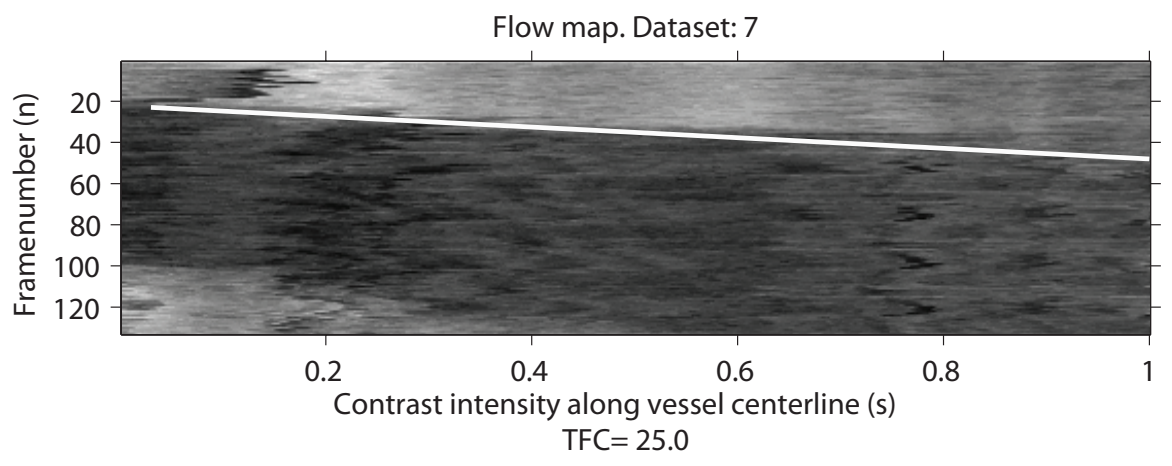

Figure 6.1: A typical flowmap.

3-d model of the coronary arteries can automate the manual point selection. In this thesis, we will focus on the creation of a 3 -d model of the coronary arteries using 2-d images. Two methods will be proposed here. First we will discuss epipolar point matching and secondly B-spline modeling in 3-d space.

\subsection{Epipolar point matching}

2-d coronary X-ray images provide information about lesions in the coronary arteries or more specific stenosis by opacifying the coronary arteries using a contrast agent. We can also use these images to estimate the functional behavior of the coronary arteries by calculating the coronary flow velocity. To do this, we have to accurately find the vessels in the images. A 2-d projection of a three dimensional object like the coronaries of the heart, likely results in overlapping and foreshortening of vessels. 3-d model creation from these 3 - $\mathrm{d}$ images can help to seperate these vessels. Research on 3 -d reconstruction of the coronary artery tree is performed by Chen et al.[3] and Blondel et al.[4] While Chen used manual feature selection, Blondel has a method which incorperates a deformation model and searches for a global minimum which should result in the most perfect 3 -d reconstruction. Messenger et al.[5] validates that 3 -d reconstructuction is usefull for obtaining accurate measurements like vessel length. A near perfect reconstruction will allow us to measure the TFC from the 2-d images using a backprojection from the 3 - $\mathrm{d}$ model.

\subsubsection{Methods}

Clinical practice at this moment to quantify the coronary flow is to use TIMI frame counting (TFC). The physician virtually places a Region of Interest (ROI) at the entry of the coronary artery and a ROI at the end of the artery. The number of frames is counted from entering of the contrast agent untill the 
contrast agent reaches the second ROI. The resulting number of frames converts to a TIMI flow grade. When measuring the TFC in hyperemic and basal state we can also define a ratio for the coronary flow reserve.

Our algorithm closely follows the method used in clinical practice. A ROI placed at several points on the vessel is used to measure the contrast agent at each time unit. Main problem here is: where to place the ROI.

ROI placement can be done when the vessel location is known. The algorithm to perform this localization exists of four parts. The first part is the 2-d image analysis which is neccessary to find the vessel features. The second part is the 3 -d reconstruction from the found vessel features. The third part will be the creation of a time model of the coronary arteries and the last part will be the backprojection of the model to the 2 -d images.

- Vessel detection Finding the coronary arteries in the 2-d images is done by an algorithm which uses the Hessian eigenvalues and multiscale filtering. A vesselness function is implemented as described in Frangi et al. [6]. Thresholding the vesselness image and skeletonizig using morphological operations results in an approximation of the vessel centerlines. This is applied to all frames in the X-ray movie.

- 3-d reconstruction Computer vision techniques are used to perform the 3 -d reconstruction. A fundamental matrix is created using the parameters found in the DICOM file. This fundamental matrix maps a point in one plane to an epipolar line in the second plane. Estimation of the fundamental matrix is critical and will be discussed in the results. Each point found in the vessel detection part of the algorithm used to select candidates in the other image. The candidates are choosen as the vessel centerline points which are closest to the epipolar line. These candidates are also calculated using a third projection. From all candidates a $3-\mathrm{d}$ point is created. Then the distance between the 3 -d reconstructed points from the first and second set of candidates is calculated. The points statisfying a minimal distance are considered as valid 3 -d points belonging to a vessel centerline. The algorithm is comparable to algorithms used by others $[3,4,7]$.

- Temporal 3-d model A temporal model of the vessel segments must be created. This subject at this moment is work in progress. The 2-d case tracking model will be shown in the results.

- Re-projection to 2-d With a temporal 3-d model of the coronary arteries we can simulate an x-ray projection. This process, called re-projection, is useful for the creation of a 2-d map of the location of coronary arteries. This map will be registered to the image from which we want to know the contrast density. 


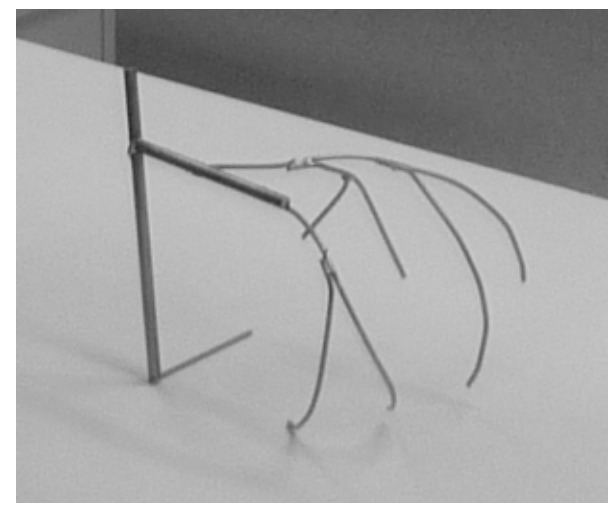

Figure 6.2: Brass coronary phantom

Acquisition in clinical practice introduces motion and calibration problems. For example table motion: To enhance image quality, physicians zoom into the area where the catheter is. While injecting the contrast agent, they follow the contrast untill they reach the end of the coronary artery by moving the table. This table motion during a movie acquisition must be converted into a correct camera positioning while doing a 3-d reconstruction. We assume that all parameters are known and motion is zero.

A computer simulated vessel phantom and a physical brass phantom is used to test the algorithm. Figure 6.2 shows our brass vessel phantom.

The brass-phantom is imaged with a C-arm using various angles. Four of these angles are selected and shown in Fig. 6.5. Manually annotation of the bifurcations and endpoints is done to be sure which points match across the images.

Three dimensional reconstruction is a well known problem in computer vision [7] and the accuracy of a 3-d point reconstruction is depending on the algorithm used. Reconstructing 3-d coronary arteries is quite a challenge. First, finding feature points is limited to the properties of a vessel: location, width, intensity, curvature, direction and bifurcation. Many algorithms rely on bifurcation detection and matching as a starting point, most of them use manually selected features. Secondly, camera calibration requires parameters which can partially be obtained from the DICOM-data: the two rotation angles, the distance source to detector and intensifier size. Patient and table motion, however, introduce translations of the camera which are difficult to estimate, but required for a good reconstruction. Finally, cardiac motion is another free parameter. Since image acquisition rates may vary e.g. 12.5, 15, 25, 30 or 50 frames per second, the cardiac phase may not be the exactly the match the phase from a latter acquisition. This introduces errors in the reconstruction if not correctly interpolated between two consequtive frames. 


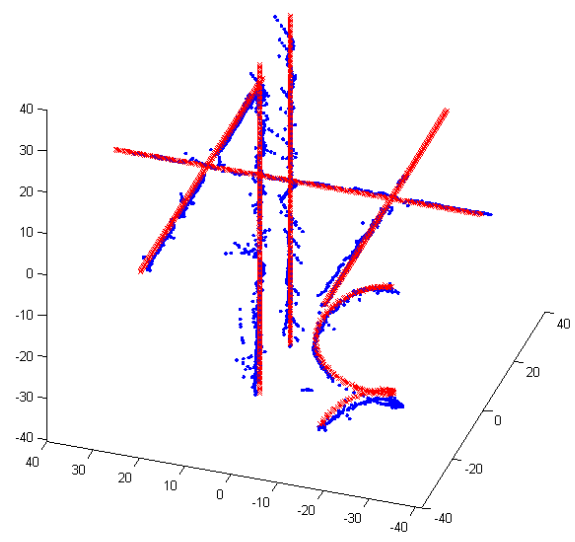

Figure 6.3: Computer simulated phantom containing several lines and one spiral. The red crosses show the ground truth and the blue dots the reconstruction.
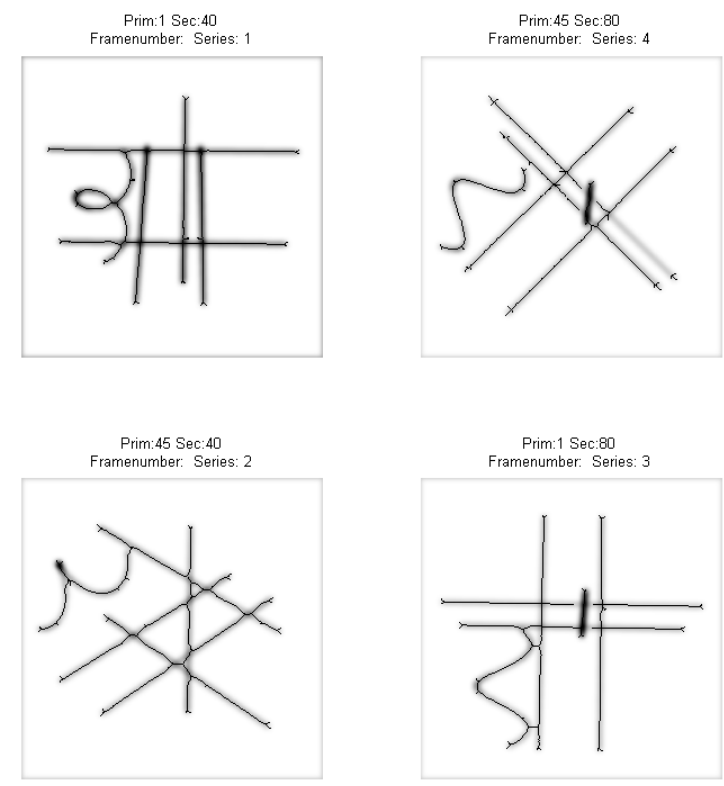

Figure 6.4: Projections made from the computer phantom, the centerline found by the vessel detection algorithm is superimposed with a black line. 

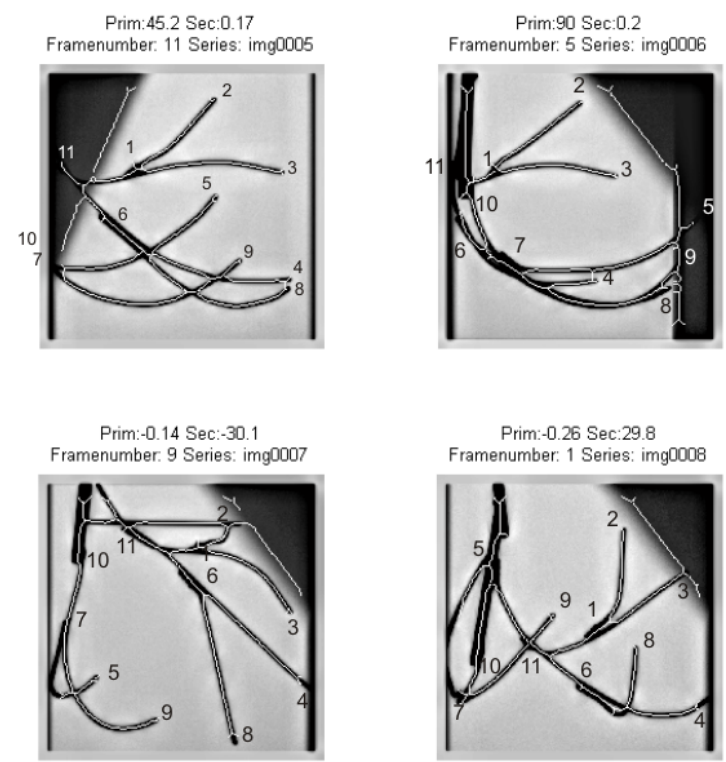

Figure 6.5: Image sequence obtained from the phantom at various angles using X-ray C-arm. The white lines represent the centerline found by our vessel detection algorithm.

In Fig 6.3 our computer simulated phantom and its 3-d reconstruction are shown. The blue dots show the reconstruction results. These results are created by making 2 -d projections of the ground truth (in red crosses), then applying the vessel detection algorithm and finally creating a 3-d reconstruction. Results from the 3 -d reconstruction of a computer simulated phantom show that our implementation of the reconstruction algorithm is able to reconstruct $3-\mathrm{d}$ test structures from 2-d images. However, the quality of the reconstruction strongly depends on the projection angles and accuracy of the vessel detection algorithm.

To compare the results of our automatic reconstruction algorithm on the brass phantom we also plotted the manual reconstruction. The reconstruction using the manually selected points is shown in red in Fig. 6.6. The manual reconstruction is based on 11 points, these are shown in Fig. 6.5, and the interconnections between the points are drawn with straight lines. The automatic reconstruction result is shown in yellow. The results are completely based on the parameters obtained from the DICOM information. Since these parameters are not $100 \%$ accurate, we have difficulties reconstructing the 3 -d information. 


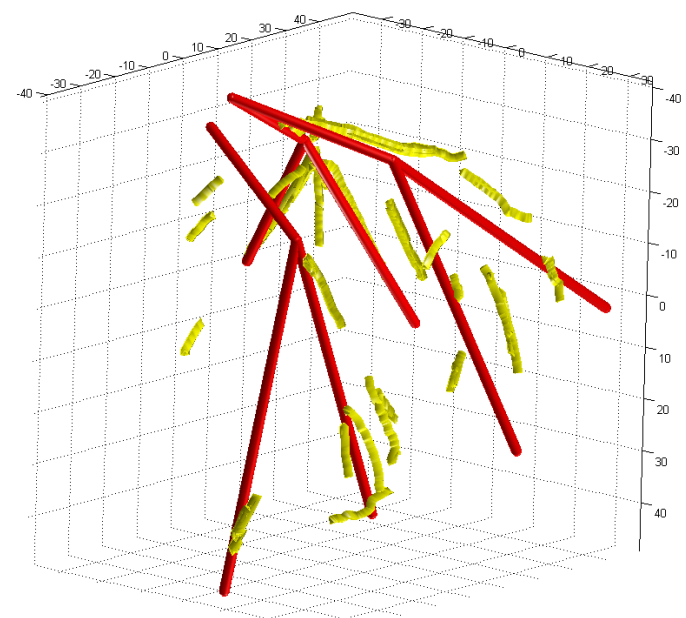

Figure 6.6: Reconstruction result of the physical phantom. In red, the manually labeled sparse reconstruction and in yellow the automatic reconstruction result using only epipolar line point matching.

\subsubsection{Discussion}

Implementation of automatic point matching using epipolar geometry appears to be quite challenging. Unknown calibration parameters make point matching inaccurate and impractical. Also point matching may result in multiple reconstructed points due to ambiguity originating from the two plane reconstruction. Clustering points, however, may result in better reconstructions. Time restrictions limit research at this point, therefore in the next section we will use annotation instead of automatic point matching.

\subsection{B-spline modeling}

Canero et al. [8] proposed a method for 3-d reconstruction using bi-plane snakes or B-splines. In our algorithm we are also using a B-spline parameterization of vessel segments, but we use a different feature map for cost minimization. The geometry of the acquisition device can be descibed by a pinhole camera model. The transformation from world $\mathbf{X}_{w}=\left[x_{w}, y_{w}, z_{w}\right]$ to camera coordinates $\mathbf{X}=$ 
$[x, y, z]$ can be described by

$$
\mathbf{X}=[\mathbf{R} \mid \mathbf{t}]\left[\begin{array}{c}
x_{w} \\
y_{w} \\
z_{w} \\
1
\end{array}\right]
$$

where $[\mathbf{R} \mid \mathbf{t}]$ are the extrinsic camera parameters in which $\mathbf{R}$ is a rotation matrix and $\mathbf{t}$ a translation vector.

The perspective projection is described by the intrinsic parameters as matrix $\mathbf{K}:$

$$
\mathbf{K}=\left[\begin{array}{ccc}
f \kappa & 0 & u_{0} \\
0 & f \kappa & v_{0} \\
0 & 0 & 1
\end{array}\right]
$$

Now we can obtain a projection using the camera position:

$$
\mathbf{X}_{p}=\mathbf{K}[\mathbf{R} \mid \mathbf{t}]\left[\begin{array}{c}
x_{w} \\
y_{w} \\
z_{w} \\
1
\end{array}\right]
$$

in which $\mathbf{K}[\mathbf{R} \mid \mathbf{t}]$ is the camera matrix $P$.

$$
\mathbf{X}=\phi^{-1}\left(\mathbf{X}_{1}, \mathbf{X}_{2}, \mathbf{P}_{1}, \mathbf{P}_{2}\right)
$$

$\phi^{-1}$ is the retroprojection operator. This operator reconstructs a three dimensional point from two given points $\mathbf{X}_{n}$ in two projection planes $n=1,2$ described by camera matrix $\mathbf{P}_{n}$. The method solves the triangulation problem using the direct linear transform. This method is described in detail in Hartley and Zisserman [7].

We will use this function to describe the force required for the deformation of the bi-plane snake. The snake is parameterezid by a 3 -d curve $\nu(s)$, where $s=[0 . .1]$ equispaced on the 3 -d curve. The deformation is controlled by an energy minimization function:

$$
E(\nu)=\int_{0}^{1}\left(E_{\text {int }}(\nu)+E_{\text {ext }}(\nu)\right) d s
$$

where $E_{\text {int }}(\nu)$ is the internal energy and $E_{\text {ext }}(\nu)$ is the external energy. The internal energy preserves smootheness and the external energy attracts the snake to image features. $E_{\text {int }}$ can be described by:

$$
E_{\text {int }}(\nu)=\alpha\left|\frac{\partial \nu}{\partial s}\right|^{2}+\beta\left|\frac{\partial^{2} \nu}{\partial s^{2}}\right|^{2}
$$

where $\alpha$ and $\beta$ are constants controlling the tension and rigidity respectively. 
The external energy $E_{\text {ext }}$ can be described by:

$$
E_{\text {ext }}(\nu)=\phi^{-1}\left(\mathbf{q}_{1}-\nabla \mathbf{F}_{1}\left(\mathbf{q}_{1}\right), \mathbf{q}_{2}-\nabla \mathbf{F}_{2}\left(\mathbf{q}_{2}\right), \mathbf{P}_{1}, \mathbf{P}_{2}\right)-\mathbf{X}
$$

where $\mathbf{q}_{n}$ are the projections of $\nu$ on view $n=1,2$ and $\mathbf{F}_{n}$ is the used feature map. The numerical implementation of the snake algorithm requires approximation of the derivatives with finite differences and converting to vector notation with $\nu_{i}=\left(x_{i}, y_{i}, z_{i}\right)[9]$ :

$$
\begin{aligned}
E_{i n t}(\nu)=\alpha \mid \nu_{i}- & \left.\nu_{i-1}\right|^{2}+\beta\left|\nu_{i-1}-2 \nu_{i}+\nu_{i+1}\right|^{2} \\
\alpha\left|\frac{\partial \nu_{i}}{\partial s}\right|^{2} & \approx \\
& =\left(\nu_{i}-\left.\nu_{i-1}\right|^{2}\right. \\
& +\left(y_{i}-y_{i-1}\right)^{2} \\
& +\left(z_{i}-z_{i-1}\right)^{2} \\
& \\
\beta\left|\frac{\partial^{2} \nu_{i}}{\partial s^{2}}\right|^{2} & \left|\nu_{i-1}-2 \nu_{i}+\nu_{i+1}\right|^{2} \\
= & \left(x_{i-1}-2 x_{i}+x_{i+1}\right)^{2} \\
+ & \left(y_{i-1}-2 y_{i}+y_{i+1}\right)^{2} \\
+ & \left(z_{i-1}-2 z_{i}+z_{i+1}\right)^{2}
\end{aligned}
$$

Notice that the distance between points is not present in these formulas, because the points are redistributed so that they are equispaced in 3-d (Euclidian distance). The redistribution of the points is done by cubic B-spline interpolation. Discretization of the integral in equation 6.5 gives:

$$
E=\sum_{i=0}^{n-1}\left(E_{\text {int }}\left(\nu_{i}\right)+E_{\text {ext }}\left(\nu_{i}\right)\right)
$$

Minimization of $E$ allows us to rewrite 6.11 to be solved using dynamic programming:

$$
\begin{aligned}
E\left(\nu_{1}, \nu_{2}, \ldots, \nu_{n}\right) & =E_{1}\left(\nu_{1}, \nu_{2}\right) \\
& +E_{2}\left(\nu_{2}, \nu_{3}\right)+\ldots \\
& +E_{n-1}\left(\nu_{n-1}, \nu_{n}\right)
\end{aligned}
$$

In case of $n=5$ nodes we can calculate the minimal energy using subfunctions $s_{k}\left(\nu_{k+1}\right)$ :

$$
\begin{aligned}
s_{1}\left(\nu_{2}\right) & =\min _{\nu_{1}} E_{1}\left(\nu_{1}, \nu_{2}\right) \\
s_{2}\left(\nu_{3}\right) & =\min _{\nu_{2}}\left(s_{1}\left(\nu_{2}\right)+E_{2}\left(\nu_{2}, \nu_{3}\right)\right) \\
s_{3}\left(\nu_{4}\right) & =\min _{\nu_{3}}\left(s_{2}\left(\nu_{3}\right)+E_{3}\left(\nu_{3}, \nu_{4}\right)\right) \\
\min _{\nu_{1}, \ldots, \nu_{5}} E\left(\nu_{1}, \ldots, \nu_{5}\right) & =\min _{\nu_{4}}\left(s_{3}\left(\nu_{4}\right)+E_{4}\left(\nu_{4}, \nu_{5}\right)\right)
\end{aligned}
$$



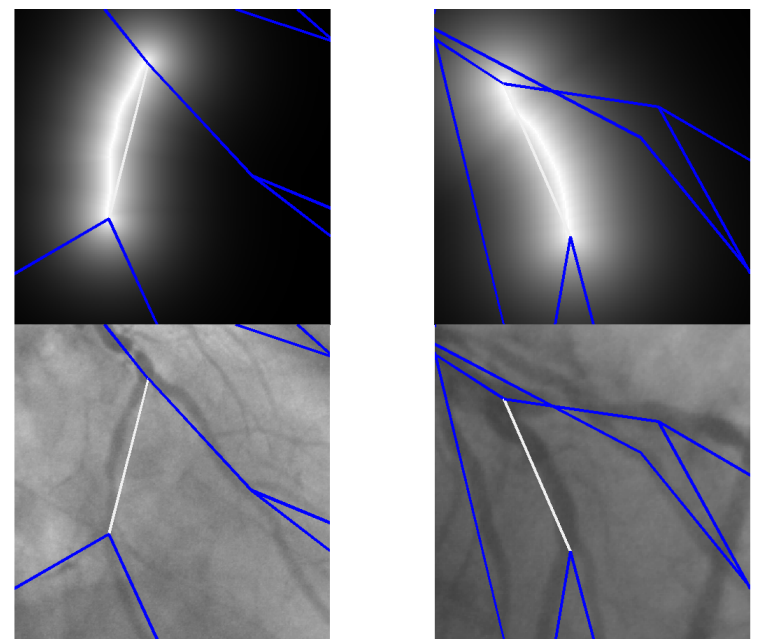

Figure 6.7: Annotation of two images at the same cardiac phase. A vessel segment is highlighted: on top the view of the feature map calculated using FMM and minimal cost path, at the bottom, the original image.

The recurrence relation is now stated as (omitting the second order term):

$$
\begin{aligned}
s_{k}\left(\nu_{k+1}\right) & = \\
& \min _{\nu_{k}}\left\{s_{k-1}\left(\nu_{k}\right)+E_{\text {ext }}\left(\nu_{k}\right)+\left|\nu_{k+1}-\nu_{k}\right|^{2}\right\}
\end{aligned}
$$

Each stage consists of 27 possibilities, the neighbors [3×3 3 ], to compute. The internal energy is stored at each stage. The indices of node position with the minimum energy cost are stored in the position matrix. The minimum energy can now be found by backtracing in the position matrix.

\subsubsection{Feature map}

The feature map is specifically constructed for each vessel segment. This is to prevent interference from other vessel segments during the minization process of the 3-d snake. Since we use annotation for the begin and endpoint of each vessel segment, we can generate a feature map from each segment. The feature map consists of a vessel centerline trace using a minimal cost path algorithm in a landscape generated by the Fast Marching Method. From the map with only the vessel centerline, a (Euclidean) distance map is calculated. This distance map is used as feature map in the snake energy minimization algorithm.

The vessel centerline is found using the Fast Marching Method (FMM) [10]. The FMM is typically used to build a map containing the travel time between the startpoint and all other points using a speed function. The FMM solves 

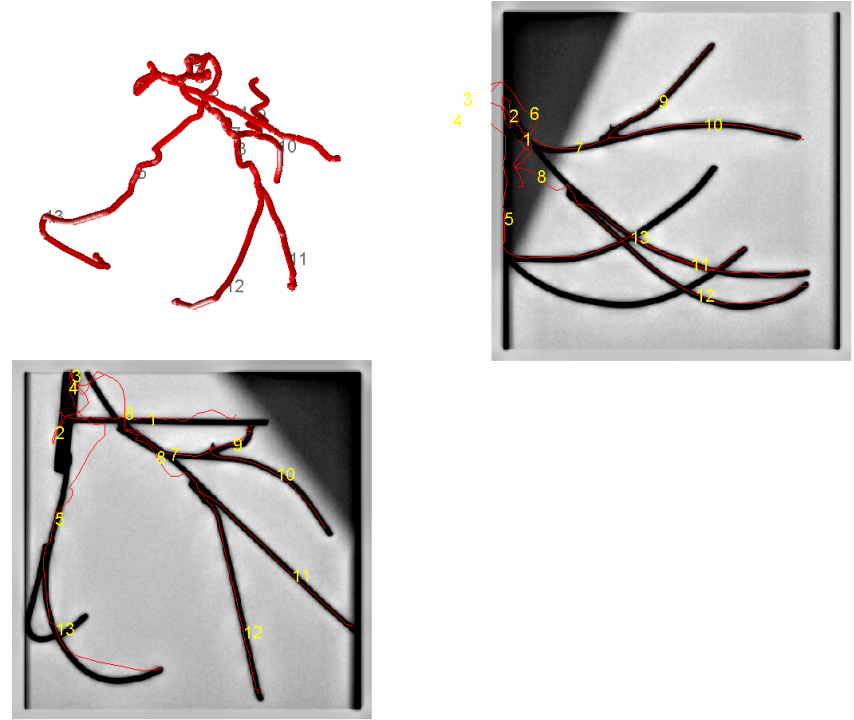

Figure 6.8: Reconstruction of phantom. See table 6.1 for the reconstruction errors.

\begin{tabular}{l|rlr|rrr} 
segment & $\mu_{1}$ & & $\sigma_{1}$ & $\mu_{2}$ & & $\sigma_{2}$ \\
\hline 1 & 8.21 & \pm & 8.81 & 78.06 & \pm & 37.27 \\
2 & 10.98 & \pm & 8.35 & 5.25 & \pm & 3.33 \\
3 & 39.10 & \pm & 32.98 & 4.41 & \pm & 2.13 \\
4 & 64.21 & \pm & 48.23 & 6.29 & \pm & 6.18 \\
5 & 8.75 & \pm & 8.73 & 2.26 & \pm & 1.10 \\
6 & 35.94 & \pm & 31.19 & 14.88 & \pm & 7.75 \\
7 & 3.65 & \pm & 2.56 & 87.46 & \pm & 58.22 \\
8 & 6.89 & \pm & 5.30 & 57.99 & \pm & 33.45 \\
9 & 2.37 & \pm & 1.40 & 1.85 & \pm & 1.05 \\
10 & 2.06 & \pm & 0.96 & 2.57 & \pm & 1.36 \\
11 & 2.94 & \pm & 1.60 & 3.68 & \pm & 2.06 \\
12 & 3.24 & \pm & 1.95 & 3.83 & \pm & 2.08 \\
13 & 7.62 & \pm & 6.59 & 3.72 & \pm & 1.84
\end{tabular}

Table 6.1: Errors in reconstruction of the phantom. See figure 6.8 for the segment locations.

the Eikonal equation:

$$
\|\nabla T(x, y)\| F(x, y)=1
$$

in which $T(x, y)$ is the arrival time of the front and $F(x, y)$ is the speed function. The speed function used as input for the Fast Marching method is based on the contrast enhanced image $I_{c}(x, y)$ :

$$
F(x, y)=\left(1-\left(G_{\sigma} \otimes I_{c}\right)\right)^{\gamma}
$$

Now the vessel centerline is the minimum cost path from the starting point 
to the end point. The centerline is defined by $\nu(s)=(x(s), y(s))$ where $x$ and $y$ are the coordinate functions and $s \in[0,1]$ is the parametric domain which discribes the vessel from start to endpoint.

\subsubsection{Calibration}

Calibration is required since the two projections are not acquired simultaneously and table motion may occur between successive acquisitions. In this case, we are relying on the position of the catheter endpoint. The position of the catheter is reconstructed in 3-d space using the catheter endpoints in both projections. The accuracy of this reconstruction is determined by the distance between the two lines originating from the 2-d plane to the focal spot. By minimizing the distance between these lines, we obtain a better reconstruction. In this case, we can alter this distance by using the $x$ and $y$ parameter of the translation $\mathbf{t}$ vector in the projection matrix. This is equal to moving the image in-plane to both $x$ and $y$ directions. The $z$ component of $\mathbf{t}$ is locked to guarantee convergence.

\subsection{Results}

Creation of a 3 -d model is required to measure blood flow. Using the method described above, we are able to create a 3 -d model using a sequence of images obtained at two different angles. The complete cardiac cycle is covered by the model. Table 6.1 shows the errors of the reconstruction results from the phantom displayed in figure 6.8 , and table 6.2 shows the errors of the reconstruction results from a clinical example displayed in figure 6.9.

\begin{tabular}{l|rlr|rrr} 
segment & $\mu_{1}$ & & $\sigma_{1}$ & $\mu_{2}$ & & $\sigma_{2}$ \\
\hline 1 & 54.34 & \pm & 29.79 & 3.25 & \pm & 1.62 \\
2 & 7.27 & \pm & 5.13 & 3.89 & \pm & 2.27 \\
3 & 2.18 & \pm & 0.96 & 4.06 & \pm & 3.58 \\
4 & 2.25 & \pm & 1.13 & 3.14 & \pm & 3.26 \\
5 & 2.30 & \pm & 1.36 & 3.90 & \pm & 2.92 \\
6 & 3.79 & \pm & 1.58 & 7.31 & \pm & 5.36 \\
7 & 4.25 & \pm & 3.25 & 16.99 & \pm & 11.56 \\
8 & 9.25 & \pm & 6.28 & 12.56 & \pm & 13.60 \\
9 & 7.24 & \pm & 7.43 & 3.07 & \pm & 1.91 \\
10 & 3.60 & \pm & 1.88 & 6.54 & \pm & 6.63 \\
11 & 2.74 & \pm & 1.46 & 4.99 & \pm & 6.21 \\
12 & 12.00 & \pm & 10.90 & 7.17 & \pm & 8.80
\end{tabular}

Table 6.2: Reconstruction errors of an clinical example case. See figure 6.9 for the segment locations.

The errors are expressed as the mean distance $\mu_{n}$ with standard deviation $\sigma_{n}$ between the projection $\left(p_{i}\right)$ of the reconstruction of the segment and the 

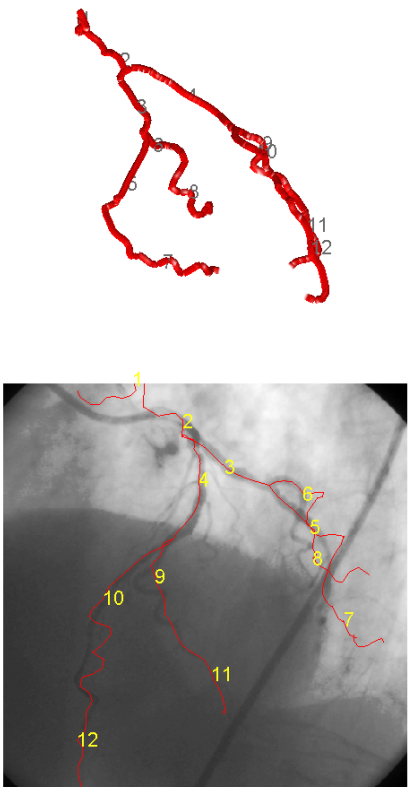

Figure 6.9: Reconstruction of a clinical case. See table 6.2 for the reconstruction errors.

centerline of the vessel $\left(q_{j}\right)$ calculated by the FMM on plane $n=1,2$ according to equation 6.17 .

$$
E=\frac{1}{N} \sum_{i=0}^{N-1} \min _{j=0}^{M-1}\left\|p_{i}-q_{j}\right\|
$$

with $N$ the number of points in the projection of the segment and $M$ the number of points from the calculated centerline. The equation calculates the minimal distance from each point in the $p$ to all points in set $q$ and calculates the mean. Using this error table we can assume that segments with a small error $(\mu<5$ pixels) in both planes can be used for our model.

\subsection{Conclusion}

The reconstruction method described in this thesis delivers 3-d models from coronary arteries. Based on the error measure we discriminate between successful and unsuccessful reconstructions. The good segments are incorperated in our model used for future flow measurements. 


\section{References}

[1] G. A. ten Brinke, C. H. Slump, and C. J. Storm, "Bi-Plane x-ray coronary 3D reconstruction for flow velocity assessment.," in Proceedings of the IEEE-EMBS Benelux Chapter 2009, IEEE EMBS Benelux Chapter, (Enschede), 2009.

[2] G. A. ten Brinke, C. H. Slump, and C. J. Storm, "Digital densitometric determination of clinical relative coronary flow distributions," in Proceedings of the SPIE, 6143, International Society for Optical Engineering, 2006.

[3] S. J. Chen and J. D. Carroll, "3-D reconstruction of coronary arterial tree to optimizeangiographic visualization," Medical Imaging, IEEE Transactions on 19(4), p. 318-336, 2000.

[4] C. Blondel, G. Malandain, R. Vaillant, and N. Ayache, "Reconstruction of coronary arteries from a single rotational x-ray projection sequence," IEEE Transactions on Medical Imaging 25(5), p. 653-663, 2006.

[5] J. C. Messenger, S. Y. J. Chen, J. D. Carroll, J. E. B. Burchenal, K. Kioussopoulos, and B. M. Groves, "3D coronary reconstruction from routine single-plane coronary angiograms: Clinical validation and quantitative analysis of the right coronary artery in 100 patients," The International Journal of Cardiovascular Imaging (formerly Cardiac Imaging) 16(6), p. $413-427,2000$.

[6] A. F. Frangi, W. J. Niessen, R. M. Hoogeveen, T. W. van, and M. A. Viergever, "Model-based quantitation of 3-D magnetic resonance angiographic images," IEEE Transactions on Medical Imaging 18(10), p. $946-56,1999$.

[7] R. Hartley and A. Zisserman, Multiple View Geometry in Computer Vision, Cambridge University Press, 2000.

[8] C. Canero, F. V. no, J. Mauri, and P. Radeva, "Predictive (un) distortion model and 3-D reconstruction by biplane snakes," IEEE transactions on medical imaging 21(9), p. 1188-1201, 2002.

[9] A. Amini, T. E. Weymouth, and R. C. Jain, "Using dynamic programming for solving variational problems in vision," IEEE transactions on pattern analysis and machine intelligence 12(9), p. 855-867, 1990.

[10] J. A. Sethian, "Evolution, implementation, and application of level set and fast marching methods for advancing fronts," Journal of Computational Physics 169(2), p. 503-555, 2001. 


\section{CHAPTER 7}

\section{Flow estimation using 3-d reconstruction ${ }^{1}$}

In coronary angiography the flow can be assessed by tracking the contrast agent in the image sequence. The contrast flow is used to estimate the functional behavior of the coronary arteries using TIMI frame count (TFC). In this thesis we propose and evaluate a method towards the automation of TFC. The method creates a two dimensional map of the contrast agent in which the opacification of the vessel centerline is plotted against time. This map is used to find the velocity of the contrast agent and subsequently the TFC. The vessel centerline is obtained using the Fast Marching Method to find the minimum cost path between the catheter point and the manually selected endpoint of the vessel. Results from 22 patients show that the algorithm is able to estimate TFC correctly within the intra observer error range of \pm 5 frames compared to manual TFC if the contrast agent is completely opacifying the coronary arteries within one cardiac cycle.

\subsection{Introduction}

Thrombolysis in Myocardial Infarction (TIMI) flow grade is a valuable and widely used qualitative measure in angiographic imaging. Since the flow grades are subjective and intra observer variability can be large, Gibson et al. [2] introduced an associated quantitative method called the TIMI Frame Count (TFC). The TFC is measured by counting the number of frames between the entering of the dye in the artery until the dye reaches the end of the artery (most distal landmark, often a bifurcation). The TFC of e.g. the right coronary artery

\footnotetext{
${ }^{1}$ This chapter is published in Computers in Cardiology 2009. [1]
} 


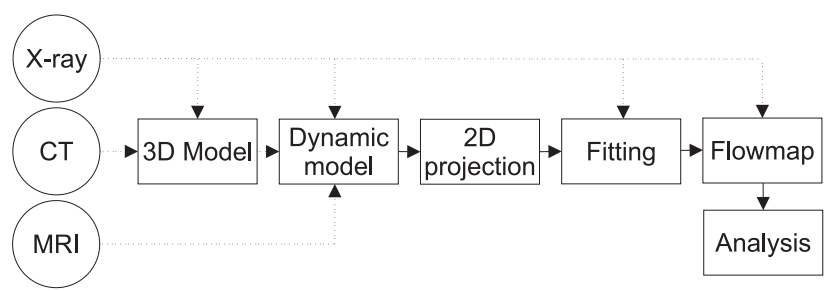

Figure 7.1: Processing stages and modalities

(RCA) is around 20 frames at $30 \mathrm{fps}$ (at other acquisition rates a conversion factor must be applied) for healthy subjects. It appears that the TFC from the RCA is comparable to the TFC from the left circumflex artery (LCx). The TFC of the left anterior descending artery (LAD) is significantly higher, therefore a correction factor of 1.7 must be applied yielding a CTFC (Corrected TFC) for the LAD. Bickel et al. [3] confirmed that the semi-quantitative TFC method is superior to the TIMI flow grading method.

When hyperemia is introduced pharmacologically using e.g. adenosine, we can measure the TFC in basal state and in hyperemic state. Division of the basal TFC (basal flow velocity index) by the hyperemic TFC (hyperemic flow velocity index) results in an estimate of the coronary flow velocity reserve. According to Stoel et al. [4], the Frame Count Reserve (FCR) and the relative FCR (rFCR) can provide a good estimate of Coronary flow Velocity Reserve (CVR) and relative Coronary flow Velocity Reserve (rCVR) and they propose the FCR as a relative simple, fast and relative inexpensive angiographic method to assess functional significance of coronary stenoses.

Although Computed Tomography Angiography (CTA) is becoming more popular, planar X-ray angiography is still the main modality for coronary intervention and the best modality for measuring contrast flow using TIMI frame counting [5]. In this thesis, we propose a method which uses 2-d X-ray images to measure the contrast flow velocity based on a similar principle as TIMI frame counting. Figure 7.1 schematically shows the processing stages and modalities usable for our method. CTA and MRI are only usable partially, while X-ray can be used in all stages of our method.

\subsection{Method}

A planar X-ray image sequence, $I(n)$, containing a conspicuous projection of the vessel during and after the injection of the contrast agent is required, with $n=[1 . . N], n$ the frame in the image sequence and $N$ the number of frames in the sequence. The recorded ECG signal is used to estimate how many frames are recorded in one cardiac cycle. All images are contrast enhanced using log-subtraction of the background. The background, $I_{b}$, is created by morpho- 
logically dilation with a disc structuring element of size $[15 \times 15]$ pixels. The cardiac cycle is subdivided into phases. From each phase we calculate a three dimensional model using annotation of the main branches in two projection. Each segment of the tree is traced in the image using a minimum cost algorithm using the Fast Marching Method (FMM) [6]. Each trace is converted to a euclidean distance map. The two distance maps are used as cost function for the 3-d spline algorithm. In our algorithm, the two traces are reconstructed in 3 - $\mathrm{d}$ space to a 3 - $\mathrm{d}$ spline, then the 3 - $\mathrm{d}$ spline is projected onto the 2 -d plane. Next, the 3-d spline is moved using a minimization criterion based on the two distance maps. The control points of the 3 -d spline are redistributed so that they are equispaced in 3-d space. A maximum of 10 iterations is used to reduce computation time. In this thesis from all images the catheter endpoint and the vessel endpoint are manually selected because we want to make sure that the startpoint and endpoint of the vessel are correct. A contrast flow map, $\Gamma(s, n)$, is created from the selected vessel [7]. The flow map consist of $N$ rows. The spatial position $s$ is the normalized length $s=[0 . .1]$ of the vessel from the start $s=0$ to the endpoint $s=1$. A typical flow map is show in figure 7.2, the temporal data $I(n)$ is plotted along the vertical axis and the spatial $s$ is plotted along the horizontal axis. Each row represents the opacity at the centerline of the vessel in which the length of the vessel is parametrised by $s$. The flow map is created using the centerline of the vessel by tracking it throughout the image sequence.

Flow map. Dataset: 7

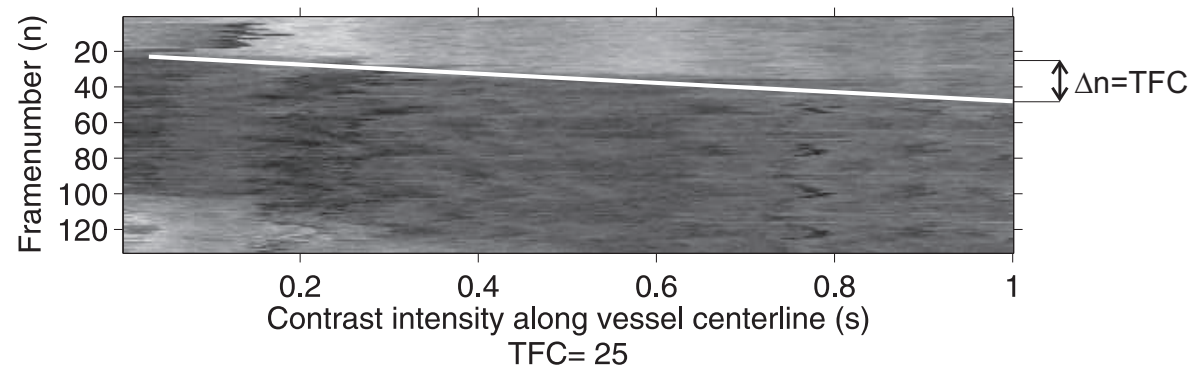

Figure 7.2: Flow map for dataset 7.

The vessel centerline is found using the FMM. The FMM is used to build a map containing the travel time between the startpoint and all other points using a speed function. The minimum cost path from the starting point to an end point can be found and is defined by $x(s)$ and $y(s)$ in which $s$ runs from the start of the vessel to the endpoint. $x(s)$ and $y(s)$ are the locations of the vessel in the image at position $s$. The FMM solves the Eikonal equation:

$$
\|\nabla T(x, y)\| F(x, y)=1
$$




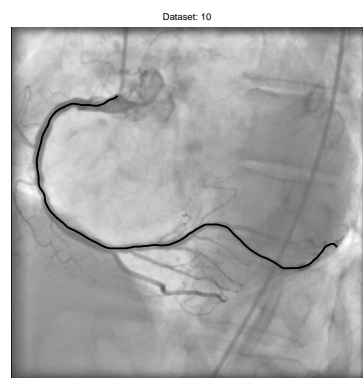

Figure 7.3: A typical vessel centerline found using the Fast Marching method. In this case only the start and end point is manually selected.

In which $T(x, y)$ is the arrival time of the front, $\|\cdot\|$ is the euclidean norm and $F(x, y)$ is the speed function. The speed function selected as input for the Fast Marching method is based on the contrast enhanced image $I_{c}(x, y)$ :

$$
F(x, y)=\left(1-\left(I_{c} \otimes G_{\sigma}\right)\right)^{\gamma}
$$

where the operator $\otimes G_{\sigma}$ is defined as Gaussian filtering with standard deviation $\sigma$. The image data is inverted so that low intensity means higher speed. Then the discrimination between low and high intensity is increased using a power of $\gamma$. In this research we have used values of $\sigma=1.5$ and $\gamma=10$. The fastest route from the startpoint to the endpoint is used as vessel centerline. A typical centerline found by this algorithm is shown in figure 7.3.

The contrast enhanced image $I_{c}$ is used to find the pixel values on the vessel centerline. The pre-contrast agent frames do not have a clear vessel centerline. For these cases we use vessel centerlines found in the frame $n+N_{p}$, in which the constant $N_{p}$ is defined as the number of frames between two $\mathrm{R}$ tops of the ECG. This centerline is written as $\mu\left(I_{c}(n), s\right)$. The data of the rows in the flow map are defined as:

$$
\Gamma(n, s)=\mu\left(I_{c}(n), s\right)
$$

Pixel values lower than the pixel values measured in the previous $N_{p}$ frame are used in the contrast flow map $\Gamma(s, n)$. The fastest path through a vessel in one frame is not always of the same length as in another frame. Therefore the whole line of pixel data is scaled using linear interpolation to the length of the contrast flow map.

The mean component is removed from all spatial components by subtracting the mean value in the temporal direction:

$$
\Gamma_{d}(n, s)=\Gamma(n, s)-\frac{1}{N} \sum_{n=1 . . N} \Gamma(n, s)
$$

Next, we calculate a threshold using a 256 bins histogram from $\Gamma_{d}$. In general, the maximum peak in the histogram belongs to the contrast agent 
(dark) values. We assume that we can seperate the opacified arteries from the transparent arteries using the value of the maximum peak position in the histogram $+1 / 8$ of the number of bins as threshold. $\Gamma_{d}$ is smoothed in temporal direction using a Gaussian with $\sigma=5$. After this, the threshold is applied. A Canny edge detector finds the lines in the contrast flow map. One line (or curve) in the contrast flow map indicates where the contrast agent enters parts of the vessel. The assumption that the contrast agent propagates with a constant velocity through the arteries allows us to use a line fit. The lines are found using the hough transform [8]. All lines that do not have a negative slope are discarded as well as lines with a starting point in the second half of the total aquisition time (we assume that the injection and full opacification of the coronary arteries happens in the first half of the recording). Evaluation of the algorithm is done by comparing the results of the TFC measurement algorithm with the manually counted TFC.
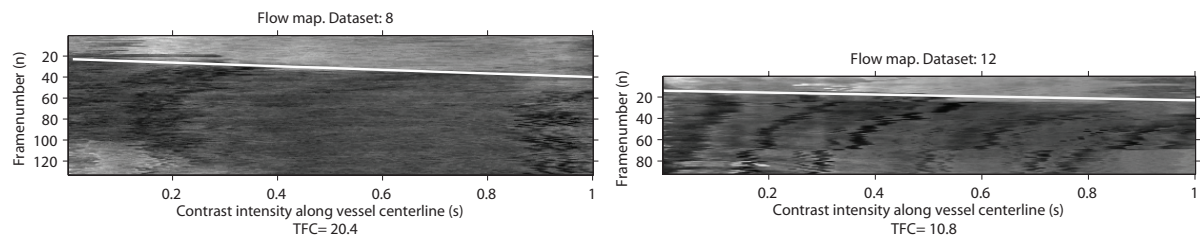

Figure 7.4: Example flow maps. Overlayed on the image is the estimated slope which correlates with the TFC.

\subsection{Experiments and results}

The method is evaluated on the coronary arteries of 22 patients from which 6 patients are imaged using a Philips Integris $\mathrm{H}(\mathrm{P})$ and 16 patients using a Siemens Axiom-Artis $(\mathrm{S}) \mathrm{C}$-arm. All the images are $[512 \times 512]$ pixels. The catheter location and the endpoint of one coronary artery are manually annotated throughout the whole image sequence by an expert. After this, the algorithm is applied. The ground truth (TFCM) was obtained by manual selection of the start and end frames by an expert. The automatic TFC (TFCA) is the output of our algorithm. The measurement is qualified as correct if the absolute difference between TFCA and TFCM is $\leq 5$, since 5 equals the intra observer difference [9]. We are working on three-dimensional modelling of the coronary arteries to assist temporal artery tracking and length quantification. Preliminary results are plotted in figure 7.5. 


\subsection{Discussion}

The proposed method can estimate the contrast flow using TIMI frame counting in 13 of 22 cases within the expected range of \pm 5 frames. The database used in this research contains images of 34 patients imaged using a Philips Integris $\mathrm{H}$ and 23 patients imaged with a Siemens Axiom-Artis. Note that the 22 cases are randomly selected from our patient database without quality preselection. In figure 7.2, a good quality flow map is drawn. It clearly shows an edge between the contrast and non-contrast region, which is also detected by the algorithm. Figure $7.4 \mathrm{~b}$ shows a flow map of the data outside the error range. There are a few reasons why the algorithm fails: Inaccurate vessel traces may occur when the selected artery is not completely in view throughout the sequence. In this case, it may happen that the boundary of the image is false identified as part of the artery. Another false tracking may occur when the catheter is clearly visible, the catheter may be tracked as part of the artery. The diaphragm can also be identified as a vessel. Since we assume that the contrast agent propagates linear throughout the vessel, the algorithm fails when we the contrast agent is not linear propagating. Most of the time this occurs when the distal part of the vessel is opacified one cardiac cycle later. This can also be seen in the flow map from dataset 12 . Furthermore, we have to take into account that we are dealing with a two-dimensional projection of a three dimensional tubular structure. Therefore, vessel structure perpendicular to the projection plane result in a higher opacity. This is clearly seen in the flow map of dataset 12 .
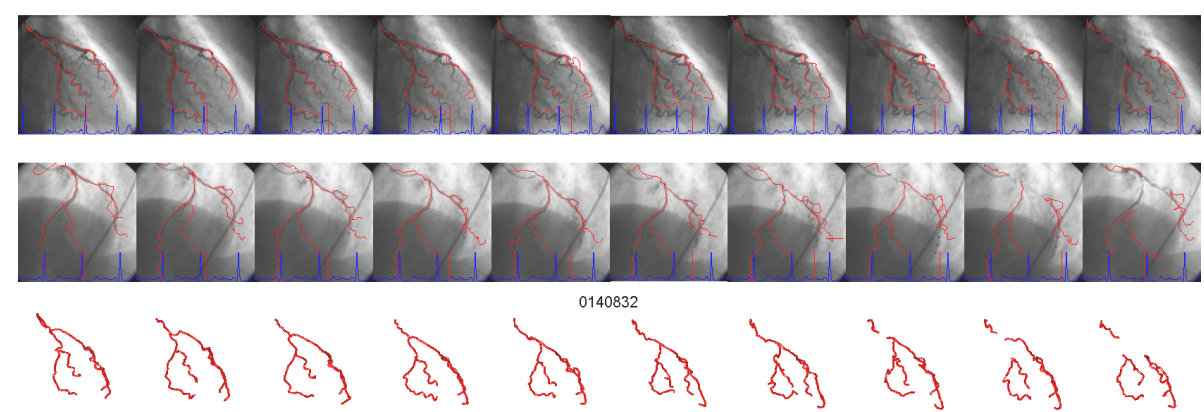

Figure 7.5: Complete cardiac cycle. The 3-d reconstruction in the bottom row is created from the top image pair.

\subsection{Conclusion}

We have presented a method towards the automation of TIMI frame counting. Our algorithm follows the procedure used in clinical practice, the exception is that we not only measure the opacification of the vessel at the begin and 
endpoint of the vessel, but along the whole vessel centerline. As far as we know, the method presented is the only computer assisted method available for TIMI frame counting. It has the potential to reduce the intra observer variability and increase the reproducability of TIMI frame counting. Our method is able to give a TFC within the error range of \pm 5 frames from 13 out of 22 randomly chosen patients. Furthermore, quantification of the coronary flow can give use more specific information about the functional impact of an apparent lesion. To do this, we have to accurately find the vessels in the images. A 2-d projection of a three dimensional object as the coronaries of the heart, likely results in overlapping and foreshortening of vessels. 3-d model creation from these $2-\mathrm{d}$ images can help to separate these vessels. Messenger et al. [10] indicate that 3 - $\mathrm{d}$ reconstruction is useful for obtaining accurate measurements such as vessel length. Our preliminary results show good reconstruction results and at this time of writing measurements with the reconstructions are in progress.

\section{References}

[1] G. A. ten Brinke, C. H. Slump, M. G. Stoel, and C. J. Storm, "A method towards automated thrombolysis in myocardial infarction (TIMI) frame counting using 3D reconstruction," 2009, p. 653-656, Computers in Cardiology, 2009.

[2] C. M. Gibson, C. P. Cannon, W. L. Daley, J. T. Dodge, B. Alexander, S. J. Marble, C. H. McCabe, L. Raymond, T. Fortin, and W. K. Poole, "TIMI frame count a quantitative method of assessing coronary artery flow," Circulation 93(5), p. 879-888, 1996.

[3] C. Bickel, H. J. Rupprecht, A. Maimaitiming, I. Welk, S. Blankenberg, F. Krummenauer, and J. Meyer, "The superiority of TIMI frame count in detecting coronary flow changes after coronary stenting compared to TIMI flow classification," The Journal of invasive cardiology 14(10), p. 590-596, 2002 .

[4] M. G. Stoel, F. Zijlstra, and C. A. Visser, "Frame count reserve," Circulation 107, p. 3034-3039, June 2003.

[5] N. E. Green, S. Y. J. Chen, J. C. Messenger, B. M. Groves, and J. D. Carroll, "Three-dimensional vascular angiography," Current Problems in Cardiology 29, p. 104-142, Mar. 2004.

[6] J. A. Sethian, "Evolution, implementation, and application of level set and fast marching methods for advancing fronts," Journal of Computational Physics 169(2), p. 503-555, 2001. 
[7] G. A. ten Brinke, C. H. Slump, and C. J. Storm, "Digital densitometric determination of clinical relative coronary flow distributions," in Proceedings of the SPIE, 6143, International Society for Optical Engineering, 2006 .

[8] J. Illingworth and J. Kittler, "A survey of the hough transform," Computer Vision, Graphics, and Image Processing 44(1), p. 87-116, 1988.

[9] J. K. French, C. J. Ellis, B. J. Webber, B. F. Williams, D. J. Amos, K. Ramanathan, R. M. L. Whitlock, and H. D. White, "Abnormal coronary flow in infarct arteries 1 year after myocardial infarction is predicted at 4 weeks by corrected thrombolysis in myocardial infarction (TIMI) frame count and stenosis severity," The American Journal of Cardiology 81, p. 665-671, Mar. 1998.

[10] J. C. Messenger, S. Y. J. Chen, J. D. Carroll, J. E. B. Burchenal, K. Kioussopoulos, and B. M. Groves, "3D coronary reconstruction from routine single-plane coronary angiograms: Clinical validation and quantitative analysis of the right coronary artery in 100 patients," The International Journal of Cardiovascular Imaging (formerly Cardiac Imaging) 16(6), p. 413-427, 2000. 


\section{CHAPTER 8}

\section{Automatic TIMI Frame counting using 3-d modeling ${ }^{1}$}

Three dimensional coronary modeling and reconstruction can assist in the quantitative analysis of coronary flow velocity from 2-d coronary images. In this thesis a novel method to assess coronary flow velocity is proposed. First, 3-d models of the coronary arteries are estimated from bi-plane $\mathrm{x}$-ray images using epipolar constraint energy minimization for the selected fiducial points like bifurcations, and subsequently 3-d Bspline energy minimization for the arterial segments. A 4-d model is assembled from a set of 3-d models representing different phases of the cardiac cycle. The 4-d model is fitted to the 2-d image sequences containing basal or hyperemic blood flow information. Then, by counting the frames in analogy with TIMI frame counting, an index of the mean coronary flow velocity can be estimated. Our experimental results show that the algorithm correlates with $r=0.98(\mathrm{P}<0.0001,95 \% \mathrm{Cl} 0.92$ to $0.99)$ to the clinical measurements of the TFC.

\subsection{Introduction}

Coronary artery disease, or more specific a stenosis, may lead to a reduction in coronary blood flow. This is manifested in a reduced flow velocity of blood through the coronary arteries. TIMI frame counting [1] is a practical method to index blood flow velocity and quantize coronary flow velocity reserve using measurements in basal and hyperemic conditions. Coronary flow velocity reserve is an important measure for heart assessment $[2,3,4]$. In clinical practice

\footnotetext{
${ }^{1}$ This chapter is submitted.
} 
the method of TIMI frame counting can be considered as a qualitative flow velocity assessment using 2-d monoplane x-ray images [5]. This method, however, is manually performed by a cardiologist and requires catheter measurements to provide information about vessel length. The standard minimally invasive modality to assess coronary arteries is mono-plane x-ray angiography, which is a two dimensional method. Three dimensional and also non-invasive methods are Computed Tomography (CT) and Magnetic Resonance Imaging (MRI). Several 3-d semi-automatic modeling methods have been proposed using monoplane [6] and bi-plane x-ray [7]. 4-d models with motion analysis are shown by Chen et al. [8] and [9]. Tomographic reconstruction techniques require multiple projection angles which are obtained in CT or rotational angiography. This requires measuring of the electrocardiogram to perform ECG-gated recording or retrospective ECG gated reconstruction. Tomographic reconstruction requires a calibrated system in which the projection geometry is well defined and at least three projections are required to get reasonable results [10]. Several improvements of this algorithm are proposed by $[11,12,13]$. Coronary models can be used, for example, in intervention planning [14] or fusion with other modalities like IVUS [15].

In this thesis we propose a method using two standard, uncalibrated, monoplane x-ray image sequences to create a 3 - $\mathrm{d}$ model of the coronary arteries. Our main research goal is to automate the measurement of mean coronary flow velocity. In our previous research [16], we have aimed at using the 2-d x-ray angiography data directly, but quantization of flow velocity requires the length of the vessel which can only be obtained using 3-d information. Furthermore our 2-d analysis required coronary model fitting in which the model ideally should be 3 -d. A set of 3-d models is created resulting in a 4 -d model of the coronary arteries covering the complete cardiac cycle. This is accomplished by creating a temporal 3-d model using the basic 2-d x-ray information acquired by standard assessment procedures. A minimum of two projection angles is required to estimate a 3 -d model from the 2 -d data, as shown in figure 8.1.

The estimated 4-d model $\mathcal{T}$ is used as a template to find the coronary arteries in the 2-d x-ray images $\mathcal{I}$. A 3-d model is selected from $\mathcal{T}$ corresponding to the normalized cardiac phase of the 2-d image. Then, a 2-d projection of the 3 - $\mathrm{d}$ model $\mathcal{M}$ is fitted onto the 2-d image by slightly adapting the 3 - $\mathrm{d}$ model. This adaptation is controlled by deforming the 3 -d model until the mean squared distance between the projection of the model wire-frame and the vessel centerlines in the $2-\mathrm{d}$ image is minimized. This process is repeated for every image in the sequence.

After a satisfactory fit of the 3-d model the contrast agent density at the location of the vessel centerline is measured. Combining the 4-d model with these measurements results in a 4-d model including information about coronary blood flow from which we can estimate coronary blood flow velocity. 


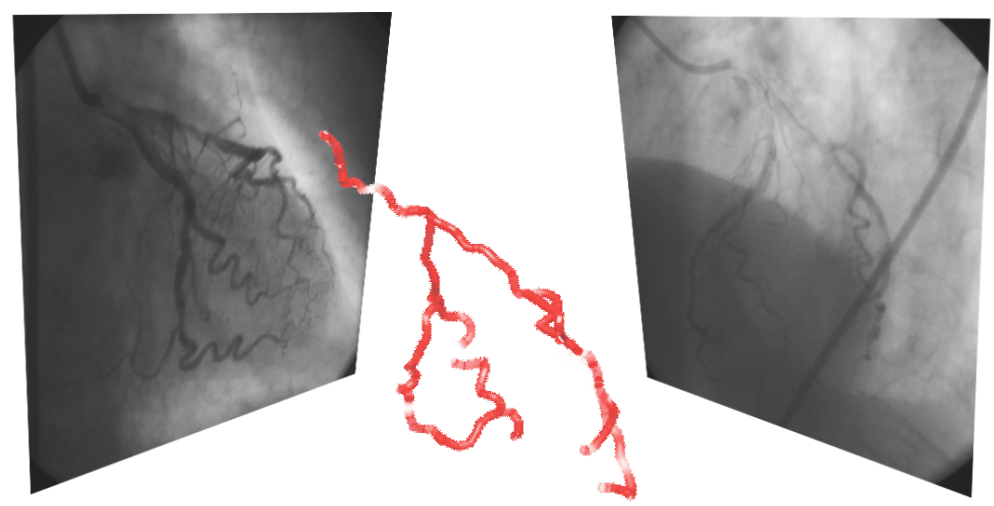

Figure 8.1: 4-d model $\mathcal{T}$ creation using mono-plane x-ray sequences at two different projection angles. The frames are selected using retrospective ECG gating. The 4-d cardiac cycle is covered by a set of 3 -d models $\mathcal{T}=\left[\mathcal{M}_{0}, \ldots, \mathcal{M}_{1}\right]$ estimated from the 2-d images $\mathcal{I}$.

\subsection{Methods}

TIMI frame counting (TFC) is a manual method to give a flow velocity index by counting the number of frames between the appearance of the contrast agent at the main trunk and at the apical bifuraction of a vessel [1]. When the blood flow velocity is artificially increased by inducing hyperemic conditions using an injection of dipyradimole, papaverine or adenosine, the frame count is in general significantly decreased. The ratio between basal and hyperemic state frame counting can be defined as the frame count reserve. Flow limiting factors, such as the presence of a stenosis, will show a limited decrease of flow velocity during the hyperemic condition compared to basal conditions. Our approach to find TFC values automatically is to extract time density curves from the all images. These time density curves are represented as an image, called a contrast flow map, which is subsequently analyzed by standard image processing algorithms. In this thesis we want to locate the vessels and measure, using densitometry, the contrast density at the vessel centerlines. Coronary vasculature is difficult to obtain from the low contrast images from only one 2 -d view. It limits the temporal analysis of a single 2-d x-ray image sequence, because the information is not sufficient to resolve the ambiguities like vessel overlap and foreshortening. Therefore, we will reconstruct a 3 -d model of the coronary arteries.

Figure 8.2 clarifies the procedure. On top are the angiographic sequences used as input to the algorithm, we firstly use two sequences, the primary and secondary angular view, to estimate a 3 -d coronary model. From the primary and secondary input sequence one cardiac cycle is selected which contains max- 


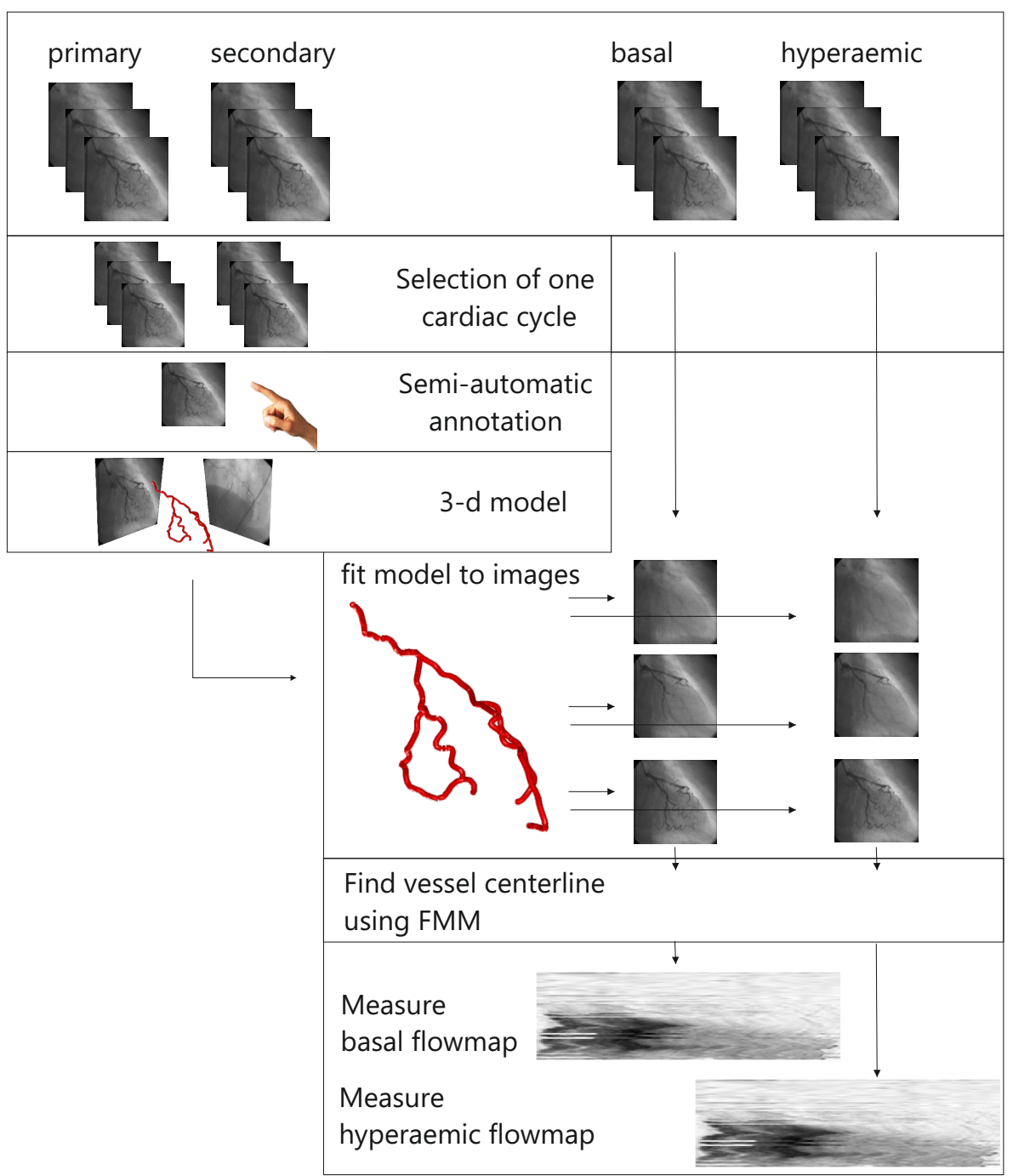

Figure 8.2: Overview of the creation of flow maps from x-ray angiography image sequences.

imum opacifaction of the coronary arteries. In these images the start and end points of the main arteries and the most important bifurcations are manually annotated in one image resulting in a set of points. Then this annotation is propagated through the remaining images using template matching, the user is able to correct the automatic annotation. The annotated sequence covering one cardiac cycle from two viewpoints is used to create a 3 -d model. This model is used to find the location of the vessels in the x-ray sequences of the 
basal and hypereamic acquisitions. The next section will explain this process in more detail.

\subsubsection{Imaging Geometry}

The 3-d coronarary reconstruction method using planar x-ray images follows the computer vision methods described by Hartley and Zisserman [17]. After the creation of the model, the model is fitted to the two dimensional image sequence using 3-d deformation and projection. Finally, a densitometric measurement results in flow maps from which we can estimate TFC. Although uncalibrated, incomplete and inaccurate, we use the information in the DICOM file to construct the geometry of the C-arm. In our case the known variables are the width of the image plane $N$ in [pixels] and the width of the intensifier $D$ in $[\mathrm{mm}]$. We assume that the focal distance $f$ in $[\mathrm{mm}]$ is equal to the distance source to detector. Based on this assumption we can calculate the detector element (pixel) size $\mu=D / N$. We also introduce a displacement vector in the image plane $\mathbf{d}=\left[d_{x}, d_{y}\right]$. Variables $f, N$ and $D$ can be extracted from the DICOM info structure from fields Distance Source to Detector, Rows and Intensifier Size, respectively. These variables define the camera calibration matrix $K$ :

$$
\mathbf{K}=\left[\begin{array}{ccc}
f \mu & 0 & \frac{1}{2} N+d_{x} \\
0 & f \mu & \frac{1}{2} N+d_{y} \\
0 & 0 & 1
\end{array}\right]
$$

The rotation matrix $R$ can be formed using the primary (RAO/LAO) and secondary angle (CAUD/CRAN) information from the DICOM info structure:

$$
\begin{aligned}
\mathbf{R}_{x}(\beta)= & {\left[\begin{array}{ccc}
1 & 0 & 0 \\
0 & \cos (\beta) & -\sin (\beta) \\
0 & \sin (\beta) & \cos (\beta)
\end{array}\right] } \\
\mathbf{R}_{y}(\alpha)= & {\left[\begin{array}{ccc}
\cos (\alpha) & 0 & \sin (\alpha) \\
0 & 1 & 0 \\
-\sin (\alpha) & 0 & \cos (\alpha)
\end{array}\right] } \\
& \mathbf{R}(\alpha, \beta)=\mathbf{R}_{\mathbf{x}}(\beta) \mathbf{R}_{\mathbf{y}}(\alpha)
\end{aligned}
$$

The primary angle corresponds to RAO $(\alpha \leq 0)$ and LAO $(\alpha>0)$ projection and the secondary angle to the CAUD $(\beta \leq 0)$ and $\operatorname{CRAN}(\beta>0)$ projection. The translation vector $\mathbf{t}$ is the position of the camera relative to the center of rotation. The z-axis points towards the ceiling, so the camera is $f / 2$ out of the center of rotation in $z$ direction:

$$
\mathbf{t}=\left[\begin{array}{c}
0 \\
0 \\
f / 2
\end{array}\right]
$$


The camera matrix is now:

$$
\mathbf{P}=\mathbf{K}[\mathbf{R t}]
$$

\subsubsection{3-d Modeling}

Fallavollita et al. [6] have proposed a method to estimate coronary arteries using snakes. Our method uses this approach with the main difference that a FMM speed-map is used as force function. The force function is important because it controls the deformation of the 3 -d snake. The snake is described by a 3-d-curve or B-spline $\nu(s)$. The deformation is controlled by an energy minimization function:

$$
E(\nu)=\int_{0}^{1}\left(E_{\text {int }}(\nu)+E_{\text {ext }}(\nu)\right) d s
$$

where $E_{\text {int }}(\nu)$ is the internal energy, preserving smoothness, and $E_{\text {ext }}(\nu)$ is the external energy, attracting the snake to image features. $E_{\text {int }}$ can be described by:

$$
E_{\text {int }}(\nu)=\gamma\left|\frac{\partial \nu}{\partial s}\right|^{2}+\lambda\left|\frac{\partial^{2} \nu}{\partial s^{2}}\right|^{2}
$$

where $\gamma$ and $\lambda$ are constants controlling the tension and rigidity of the snake respectively.

The external energy $E_{\text {ext }}$ can be described by:

$$
E_{\text {ext }}(\nu)=\phi^{-1}\left(\mathbf{x}_{1}, \mathbf{x}_{2}, \mathbf{P}_{1}, \mathbf{P}_{2}\right)-\mathbf{X}
$$

where $\phi^{-1}$ is the retro-projection operator. This operator reconstructs a 3 - $d$ point from two given 2 -d points $\mathbf{x}_{n}$ in two projection planes $n=1,2$ described by camera matrix $\mathbf{P}_{n}$ :

$$
\mathbf{X}=\phi^{-1}\left(\mathbf{x}_{1}, \mathbf{x}_{2}, \mathbf{P}_{1}, \mathbf{P}_{2}\right)
$$

$\mathbf{x}_{n}$ are the original points $\mathbf{q}_{n}$ with a movement depending on the force maps $\mathbf{F}_{n}$ :

$$
\mathbf{x}_{n}=\mathbf{q}_{n}-\nabla \mathbf{F}_{n}\left(\mathbf{q}_{n}\right)
$$

where $\mathbf{q}_{n}$ are the projections of $\nu$ on projection planes $n=1,2$ and $\mathbf{F}_{n}$ are the used force maps, which will be discussed in the next section.

The numerical implementation of the snake algorithm requires approximation of the derivatives with finite differences. Conversion to vector notation with $\nu_{i}=\left(x_{i}, y_{i}, z_{i}\right)[18]$ results in:

$$
\begin{aligned}
& E_{i n t}(\nu)=\gamma\left|\nu_{i}-\nu_{i-1}\right|^{2}+\lambda\left|\nu_{i-1}-2 \nu_{i}+\nu_{i+1}\right|^{2} \\
& \gamma\left|\frac{\partial \nu_{i}}{\partial s}\right|^{2} \approx \gamma\left|\nu_{i}-\nu_{i-1}\right|^{2} \\
&=\gamma\left(\left(x_{i}-x_{i-1}\right)^{2}\right. \\
&+\left(y_{i}-y_{i-1}\right)^{2} \\
&\left.+\left(z_{i}-z_{i-1}\right)^{2}\right)
\end{aligned}
$$




$$
\begin{aligned}
\lambda\left|\frac{\partial^{2} \nu_{i}}{\partial s^{2}}\right|^{2} & \approx \lambda\left|\nu_{i-1}-2 \nu_{i}+\nu_{i+1}\right|^{2} \\
& =\lambda\left(\left(x_{i-1}-2 x_{i}+x_{i+1}\right)^{2}\right. \\
& +\left(y_{i-1}-2 y_{i}+y_{i+1}\right)^{2} \\
& \left.+\left(z_{i-1}-2 z_{i}+z_{i+1}\right)^{2}\right)
\end{aligned}
$$

The distance between the points is kept equidistant (Euclidean distance) by redistribution of the points along the snake using cubib B-spline interpolation. Discretization of the integral in equation 8.7 gives:

$$
E=\sum_{i=0}^{N-1}\left(E_{\text {int }}\left(\nu_{i}\right)+E_{\text {ext }}\left(\nu_{i}\right)\right)
$$

for a snake with $N$ nodes.

Minimization of $E$ allows us to rewrite equation 8.15 to be solved using dynamic programming:

$$
\begin{aligned}
E\left(\nu_{1}, \nu_{2}, \ldots, \nu_{N}\right) & =E_{1}\left(\nu_{1}, \nu_{2}\right) \\
& +E_{2}\left(\nu_{2}, \nu_{3}\right)+\ldots \\
& +E_{N-1}\left(\nu_{N-1}, \nu_{N}\right)
\end{aligned}
$$

In case of, for example, $N=5$ nodes we can calculate the minimal energy using sub-functions $s_{k}\left(\nu_{k+1}\right)$ :

$$
\begin{aligned}
s_{1}\left(\nu_{2}\right) & =\min _{\nu_{1}} E_{1}\left(\nu_{1}, \nu_{2}\right) \\
s_{2}\left(\nu_{3}\right) & =\min _{\nu_{2}}\left(s_{1}\left(\nu_{2}\right)+E_{2}\left(\nu_{2}, \nu_{3}\right)\right) \\
s_{3}\left(\nu_{4}\right) & =\min _{\nu_{3}}\left(s_{2}\left(\nu_{3}\right)+E_{3}\left(\nu_{3}, \nu_{4}\right)\right) \\
\min _{\nu_{1}, \ldots, \nu_{5}} E\left(\nu_{1}, \ldots, \nu_{5}\right) & =\min _{\nu_{4}}\left(s_{3}\left(\nu_{4}\right)+E_{4}\left(\nu_{4}, \nu_{5}\right)\right)
\end{aligned}
$$

The recurrence relation is now stated as (for clarity the second order term is not presented):

$$
\begin{aligned}
& s_{k}\left(\nu_{k+1}\right)= \\
& \min _{\nu_{k}}\left\{s_{k-1}\left(\nu_{k}\right)+E_{\text {ext }}\left(\nu_{k}\right)+\left|\nu_{k+1}-\nu_{k}\right|^{2}\right\}
\end{aligned}
$$

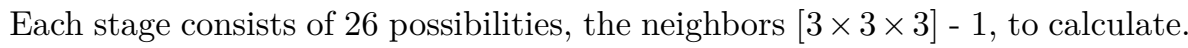
The internal energy is stored at each stage. The indices of node position with the minimum energy cost are stored in the position matrix. The minimum energy can now be found by back-tracing in the position matrix using Dijkstra's shortest path algoritm [19]. 

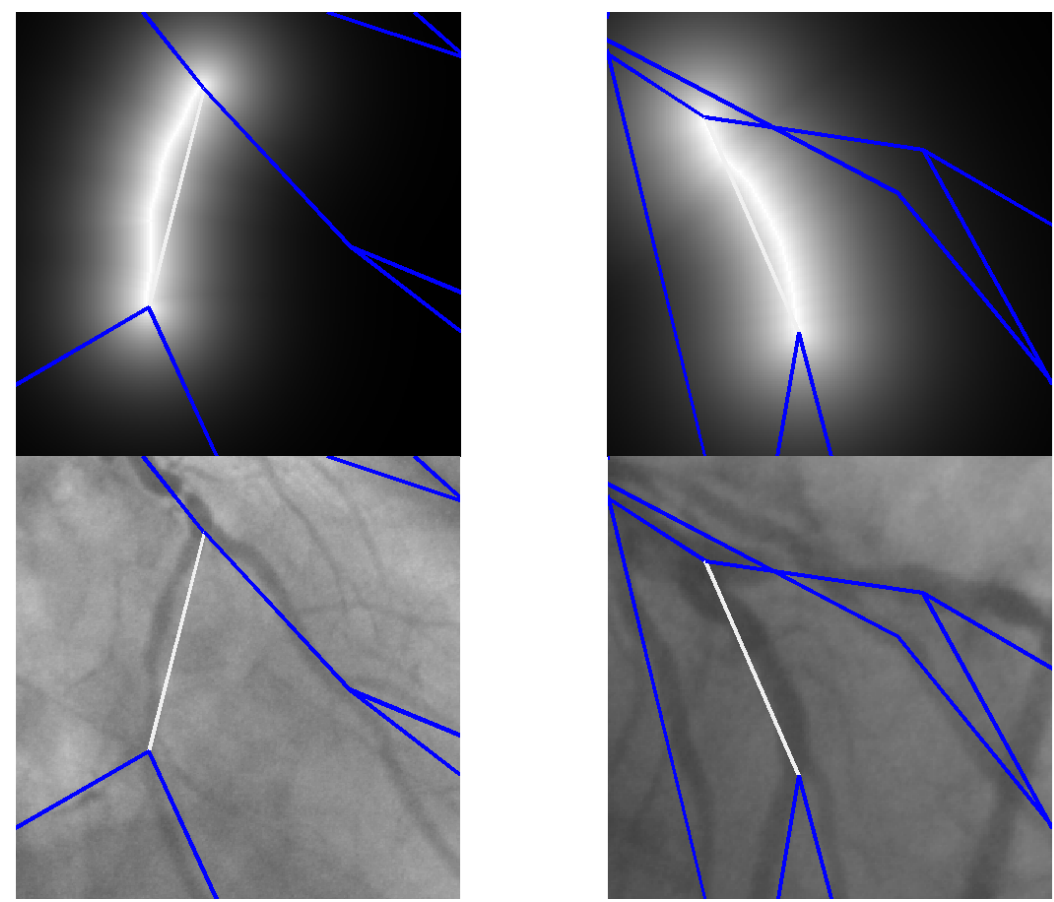

Figure 8.3: The bottom two images show the annotation of the vessel segments with a highlight (in white) of the vessel segment currently being traced. The upper figure shows the euclidean distance map after vessel detection using the FMM method and minimal cost path algorithm. The gradient of the distance map shown in the upper figures are used as force field in the snake deformation algorithm.

\subsubsection{Force map}

The force map is specifically constructed for each vessel segment to prevent interference from other vessel segments during the minimization process of the 3 -d-snake. The begin and endpoint of each vessel segment is annotated. With this annotation we can generate a force map from each vessel segment using the Fast Marching Method (FMM) [20]. This force map is used to find the vessel centerline using the minimal cost path algorithm. The 2 -d vesselcenterline is plotted in a 2-d grid. From this grid a euclidean distance map is calculated. The gradient of the distance map is used as force map in the snake energy minimization algorithm.

The vessel centerline is found using the 2-d multi-stencils fast marching method (FMM) [21]. This FMM is used to build a map containing the travel time between the start-point and all other points using a force function, which can be considered as the speed. The FMM solves the Eikonal equation:

$$
\|\nabla T(x, y)\| F(x, y)=1
$$


in which $T(x, y)$ is the arrival time of the front and $F(x, y)$ is the force function. The contrast enhanced image $I_{c}(x, y)$ is used as input $F(x, y)$ for the fast marching method:

$$
F(x, y)=\left(1-\left(G_{\sigma} \otimes I_{c}\right)\right)^{\gamma}
$$

Now the vessel centerline is the minimum cost path from the starting point to the end point, the back-tracing of the minimal cost past is performed using a fourth order Runge-Kutta approximation. The centerline is defined by $\nu(s)=$ $(x(s), y(s))$ where $x$ and $y$ are the coordinate functions and $s \in[0,1]$ is the parametric domain which describes the vessel from start to endpoint.

\subsubsection{Table motion compensation}

Table motion is compensated using image plane shifting. The only requirement for this algorithm is the annotation of the catheter-tip throughout the image sequence. The correction is performed in the $\mathrm{x}-\mathrm{y}$ plane of the local coordinate system, this results in a converging solution. Equation 8.6 is changed to include the table translation in the $\mathrm{x}-\mathrm{y}$ plane:

$$
\mathbf{P}=\mathbf{K}\left[\mathbf{R}\left[\mathbf{t}+\mathbf{R}^{-1} \mathbf{t}_{t}\right]\right]
$$

In which $\mathbf{t}_{t}$ is the vector:

$$
\mathbf{t}_{t}=\left[\begin{array}{c}
x_{t} \\
y_{t} \\
0
\end{array}\right]
$$

This vector has two parameters $x_{t}$ and $y_{t}$. These parameters represent table motion, which results in an $\mathrm{x}-\mathrm{y}$ motion in the image plane. The parameters can be solved by minimizing the re-projection error of the catheter-tip in the second image plane using equation 8.9. The method solves the triangulation problem using the direct linear transform. This method is described in detail in Hartley and Zisserman [17].

\subsubsection{Fitting}

The 3-d model is fitted to each frame in the image sequence from which we want to measure the TFC. The vessel centerline is obtained using the FMM method. The start and end points of the vessel segment are determined by projection of the segments of the 3-d model. Most likely, the projection of the segment will not fit the vessel centerline found by the FMM method. Therefore, the 3 - $\mathrm{d}$ model is iteratively deformed until the vessel centerline coincides with the projection of the segment. We allow a maximum of 10 iterations of the model deformation to prevent over-fitting when a vessel centerline is incorrectly found due to low or none contrast density. 


\subsubsection{Annotation}

Three dimensional reconstruction from a limited set of projections $\left(N_{p}=2\right)$ is a challenging task. Therefore we have created a ground truth dataset using semi automatic annotation of our dataset. The process of annotating the dataset is based on our flow estimation software as described in ten Brinke et al. 2006 [16]. We have discarded image pre-filtering with coherence filters and vessel segmentation algorithms to prevent error accumulation and experimental thresholds.

The user selects an image from the dataset with maximum opacified arteries. Next, the vessel structure is annotated by selecting bifurcations and endpoints. These points are denoted $\mathcal{P}_{i}(x, y)$. Points are interconnected using segments which are stored in a connection matrix $\mathcal{C}_{i, j}$ connecting point $\mathcal{P}_{i}$ with point $\mathcal{P}_{j}$. It is a redundant matrix, so only the upper triangle is used. All points can be labeled $\mathcal{L}_{i}$, for example 'LAD', 'LCx', 'Cathetertip'. A dataset contains the annotation set $\mathcal{S}=\{\mathcal{P}(n), \mathcal{C}, \mathcal{L}\}$ in which $n=[1 . . N]$, with $N$ the number of images in the dataset. So each image shares the same labels, interconnections and amount of points, only the locations of the points vary. This allows the creation of a temporal 3-d model using accurate data points. The user can use fast marching assistance for tracing the vessel centerline to make sure that the annotation correctly follows the vessels. All other frames are semi-automatically annotated using template matching.

\subsubsection{Contrast flow map analysis}

From the DICOM file we obtain 8-bit grayscale images with a size of $[512 \times$ 512 ] pixels. The raw data will be used to construct the contrast flow map, which results in a flow map with the same resolution as the images. Each line in the flow map represtent the contrast agent density along one vessel centerline $s=[0 . .1]$, with in the vertical direction the image index $n=[1 . . N]$, see figure 8.4. In this research we will focus on the most clearly visible vessel, which is the LAD. The contrast flow map is analyzed using the following image processing steps: The mean value is removed from the single frame measurements. This mean value $\Gamma_{\text {mean }}$ is the mean from all single measurements in the temporal direction:

$$
\Gamma_{\text {mean }}(n, s)=\Gamma(n, s)-\frac{1}{N} \sum_{n=0}^{N-1} \Gamma(n, s)
$$

for $s=[0 . .1]$. Next we calculate a threshold using a 256 bins histogram from $\Gamma_{\text {mean }}$. In general, the maximum peak in the histogram belongs to the contrast agent (dark) values. In practice, we can separate the opacified arteries from the transparent arteries using a threshold consisting of the maximum peak position in the histogram plus the standard deviation of the peak which is empirically set at $1 / 8$ of the total number of bins. 


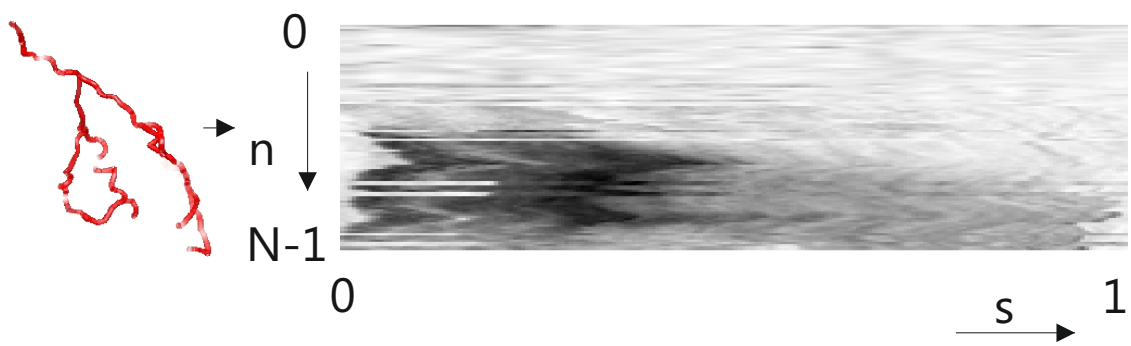

Figure 8.4: Densitometry is applied on each image. The result is the measurement of the contrast density at the location of the vessels. This information is shown in a flow-map for each vessel. From this the coronary flow velocity can be estimated.

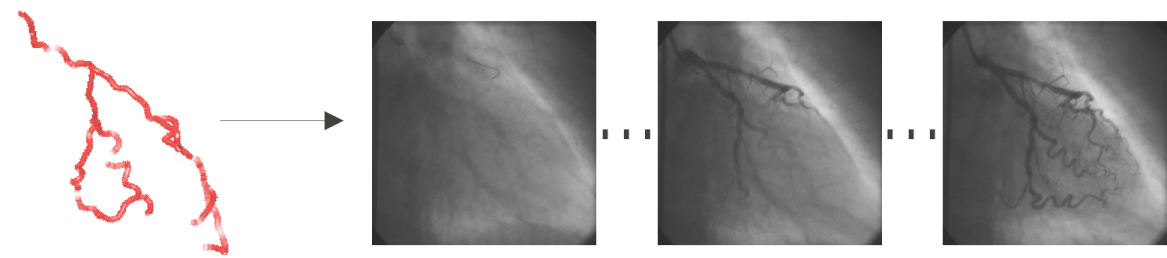

Figure 8.5: The 3-d model closest to the cardiac phase of each image is selected to be fitted to the 2-d x-ray image. This is done for each image in the hyperemic and basal image sequence. The model is aligned in each image using the catheter-end point, which is manually annotated.

This threshold is then applied to the contrast flow map. A Canny [22] edge detector finds the lines in the contrast flow map. One line (or curve) in the contrast flow map connects the arrival times and vessel positions of the contrast agent. The assumption that the contrast agent propagates with a constant velocity through the arteries allows us to use a line fit. The lines are detected using the Hough transform [23]. The slope of the line is related to the contrast flow velocity. All lines that do not have a negative slope are discarded as well as lines with a starting point after $1 / 2$ of the total acquisition time (we assume that the coronary arteries will be fully opacified by the injection of the contrast agent in the first half of the recorded image sequence).

\subsection{Experiments}

Two experiments are conducted: Using a static brass model, see figure 8.6, imaged using a Phillips Xper x-ray scanner and with clinical data.

Our clinical dataset contains images of interventions with specific TIMI frame counting data and coronary artery length measurements. The coronary 


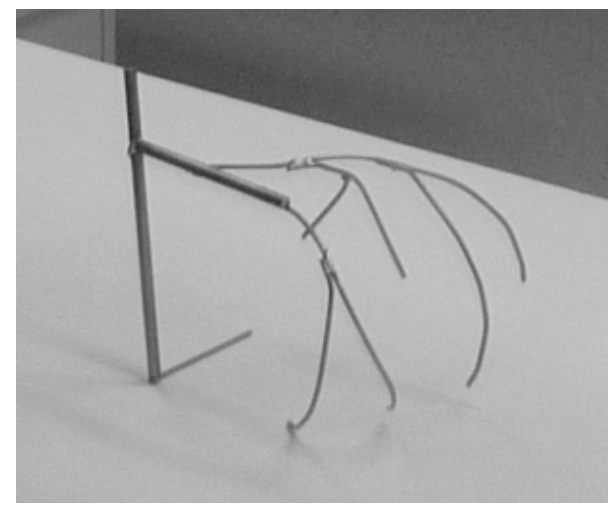

Figure 8.6: The brass coronary phantom

artery length is clinically measured using a catheter starting from the left main artery to the apical bifurcation of the LAD. It contains 32 patients (11 female, 21 male) from which two assessments are discarded because they do not contain at least two different projection angles of the left-coronary artery. From the resulting 30 patients a total of 13096 images have been semi-automatically annotated. The two visually best series are selected for annotation taking into account a large angular distance between the series, allowing to recreate a 3-d model. Note that, in constrast to the catheter measurements, in the measurements of the reconstructed coronary arteries we only measure from the start of the LAD to the apical bifurcation.

\subsection{Results}

\subsubsection{Phantom}

We have tested the 3 -d modeling algorithm on the brass phantom. A fully automatic reconstruction of the vessel segments based on epipolar line matching was not successful due to the unknown camera calibration parameters. In the literature methods are proposed by e.g. Blondel et al.[9] to address this problem. Since it is not the focus of our research to create reconstruction algorithms we have decided to manually annotate the vessel segments. With the manual annotation and the B-spline algorithm proposed by Fallavollita et al. [6] we were able to create usable models from the 2-d data. Figure 8.8 shows the reconstruction of the phantom and table 8.1 shows the errors made in the reconstruction. Large variations are visible at the vesselsegements which are not clearly visible in both images. 

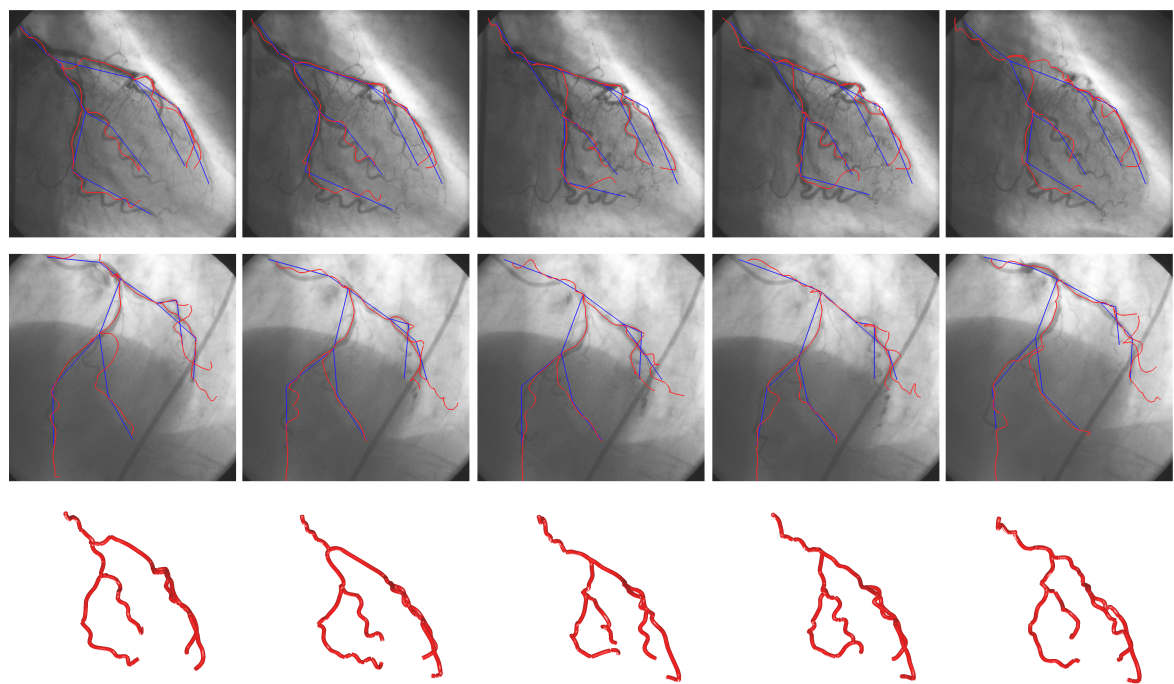

Figure 8.7: Modeling of the coronary arteries. The first and second row show the two images used in the modeling, the third row shows the generated model. A total of 20 models are created to cover the complete cardiac cycle, only 5 are shown in this figure.

\begin{tabular}{l|rl|rl} 
segment & $\begin{array}{r}\mu_{1} \\
\text { [pixels] }\end{array}$ & $\begin{array}{r}\sigma_{1} \\
\text { [pixels] }\end{array}$ & $\begin{array}{r}\mu_{2} \\
\text { [pixels] }\end{array}$ & $\begin{array}{l}\sigma_{2} \\
\text { [pixels] }\end{array}$ \\
\hline 1 & 8 & \pm 8 & 78 & \pm 37 \\
2 & 10 & \pm 8 & 5 & \pm 3 \\
3 & 39 & \pm 32 & 4 & \pm 2 \\
4 & 64 & \pm 48 & 6 & \pm 6 \\
5 & 8 & \pm 8 & 2 & \pm 1 \\
6 & 35 & \pm 31 & 14 & \pm 7 \\
7 & 3 & \pm 2 & 87 & \pm 58 \\
8 & 6 & \pm 5 & 57 & \pm 33 \\
9 & 2 & \pm 1 & 1 & \pm 1 \\
10 & 2 & \pm 0 & 2 & \pm 1 \\
11 & 2 & \pm 1 & 3 & \pm 2 \\
12 & 3 & \pm 1 & 3 & \pm 2 \\
13 & 7 & \pm 6 & 3 & \pm 1
\end{tabular}

Table 8.1: Reconstruction results of the phantom. See figure 8.8 for the segment locations.

\subsubsection{Clinical data}

The quality of the coronary models is characterized by the re-projection error. The length of the LAD is known from catheter measurements, so we will compare the LAD length measured from the model with the ground truth of the catheter measurement. The results are displayed in figure 8.9.

Overall results are depicted in figure 8.10a. The measured TFC correlates with the clinical measurements with $\mathrm{r}=0.80(\mathrm{P}<0.0001,95 \%$ CI 0.63 to 0.89$)$. 

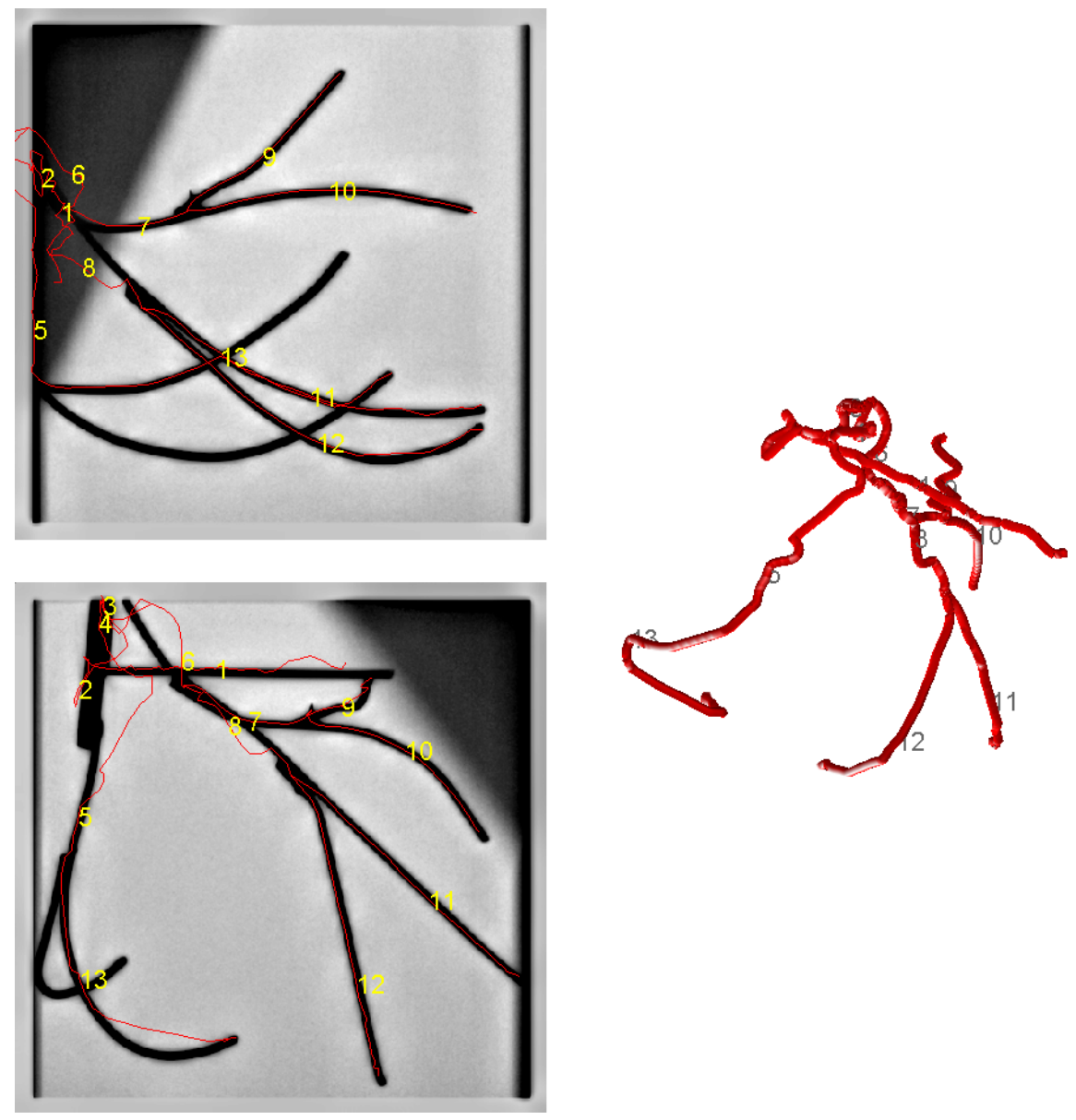

Figure 8.8: Reconstruction of phantom. See table 8.1 for the reconstruction results.

From 14 of 32 patients we have data of completely opacified coronary arteries during one cardiac cycle from two viewing angles. This data is used to create models covering the complete cardiac cycle. In figure 8.7 an example of 3 -d models created from 5 phases of the cardiac cycle are shown. Retrospective gatng is used to select two images for the creation of one 3-d model, the figure shows semi-automatically annotation of the vessels in blue and the 2-d projection of the final model in red. The 2-d projection of the model does not exactly fit the x-ray image, but that is not a main issue because the 3 -d model is deformed to fit the x-ray image in a latter phase. From 9 of these 14 patients we were able to obtain a measured TFC within the intra-observer variability range of $+/-5$ frames. The measured TFC correlates with the clinical measurements 
Measured and ground truth of LAD Length (clipped at $250 \mathrm{~mm}$ ) Ordered by 3-D snake registration error.

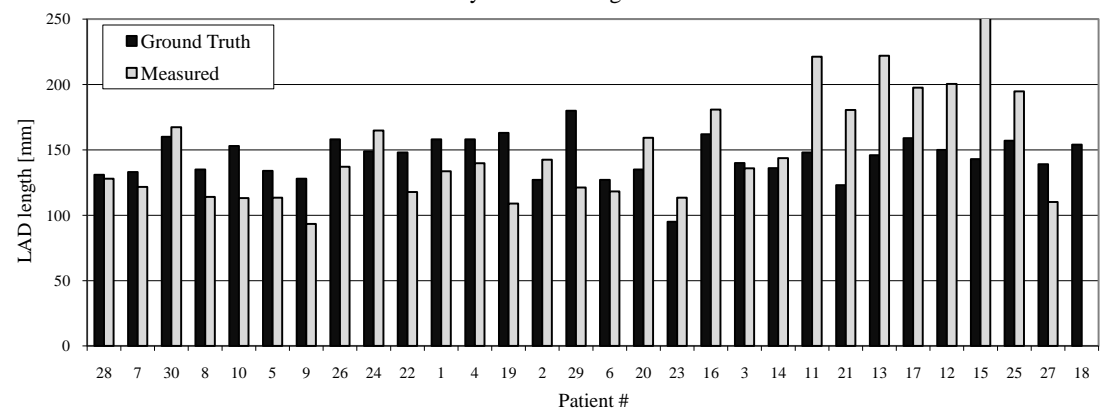

Figure 8.9: Length of the Left Anterior Descending (LAD) artery. The length calculated from the 3-d model is compared to the length measured with a catheter (ground truth). The patients are ordered by registration error. The registration error is a measure for the accuracy of the LAD length measurement.

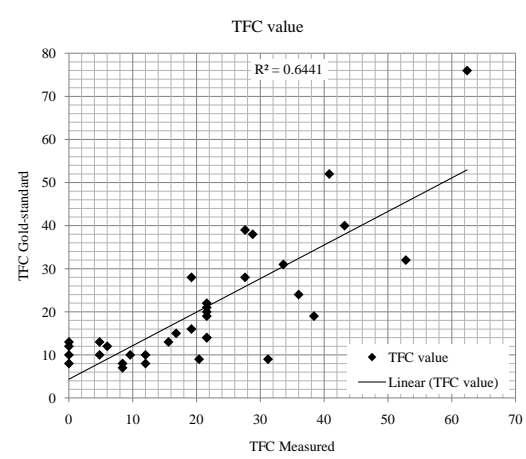

a: Scatter-plot with linear fit of the measured and ground truth TFC values of the 32 patients.

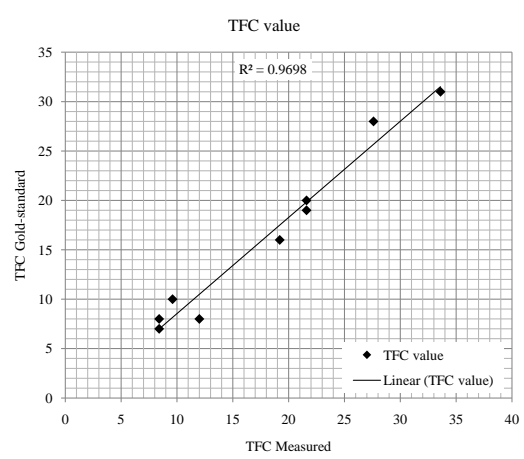

b: Scatter-plot with linear fit of the measured and ground truth TFC values of the remaining 9 patients.

Figure 8.10: Scatter-plots showing the correlation between the measured (using 3-d modeling and flow measurements) and the ground thruth (measurements performed by a cardiologist)

with $\mathrm{r}=0.98(\mathrm{P}<0.0001,95 \%$ CI 0.92 to 0.99$)$. See figure $8.10 \mathrm{~b}$.

\subsection{Discussion}

The accuracy of the algorithm depends on clinical and technological limitations. Acquisition of high quality images is limited by the amount of x-ray exposure to the patient and the clinician. Therefore, the clinician tries to 
mimize the x-ray dose by highly effective usage of the field of view. This results in table motion during the acquisition and a minimum amount of images. Also, toxic effects of contrast agent limit the amount of image aquisition runs. Dose limitation results in lower quality images or incomplete opacification of the coronary structure. Furthermore, the health condition of the patient may limit the usage of the breath hold technique. Moreover, patient arrhythmias during acquisitions may occur spontaneous or due to the injection of contrast agents or vasodilators. Finally, patient dimensions influences the amount of $\mathrm{x}$-ray required and as a result changes the brightness of the images. This may vary at different acquisition angles.

The clinical acquisition problems immediately have an effect on the performance of the analysis software: For 3-d modeling, a calibrated x-ray system is required. System calibration allows epipolar matching and quantized models. Also, the creation of a temporal model requires one completely opacified coronary vessel structure during the whole cardiac cycle. For 3-d modeling it also requires to have this from a minimum of two different angles. Ambiguity still exists, but can be reduced using the B-spline models of the vessel segments. Patient motion like breathing and table motion result in motion artifacts. The results of these motions are inaccurate models. We have minimized these errors by annotating the catheter-tip and applying motion correction by aligning the catheter-tips in both imaging planes.

During an x-ray acquisition, several image enhancements may affect the analysis software. Automatic exposure control, for example, results in an extra variable which should be included in densitometric measurements. This variable, however, is not updated during the acquisition. Anatomical background structures, like the spinal cord, influence vessel tracing algorithms. Theoretically we can use a background subtraction algorithm, in practice, motion artifacts prevent its application. In this research 3-d modeling is used to compensate for that.

\subsubsection{Limitations}

The method discussed in this chapter can only be used when at least two acquisitions are available with a complete opacification of the coronary arteries during one complete cardiac cycle. The time resolution of the measurements is limited by the frame-rate of the x-ray equipment. In normal acquisitions this frame-rate is 12.5 or 15 frames per second (fps), resulting in respectively $80 \mathrm{~ms}$ or $66.7 \mathrm{~ms}$ maximum temporal resolution (without interpolation) depending on the acquisition device. Acquisitions with $50 \mathrm{fps}$ are technically no problem, however, clinically not desired because of higher x-ray exposure to patient and clinician. 


\subsubsection{Conclusion}

In this thesis we have proposed a method towards the automation of TIMI frame counting and, as a result of that, the measurement of coronary flow velocity reserve using standard angiographic x-ray acquisition. It requires a full cardiac cycle of completely opacified coronary arteries from at least two different angular positions recorded with ECG and an additional run for the hyperemic measurement.

From 9 patients we were able to obtain a measured TFC within the intraobserver variability range of $+/-5$ frames. The measured TFC correlates with the clinical measurements with $\mathrm{r}=0.98(\mathrm{P}<0.0001,95 \%$ CI 0.92 to 0.99$)$.

\subsubsection{Acknowledgments}

The authors thank M. Eskes BSc and P. Gooskens BSc for annotation of the dataset used in this research.

\section{References}

[1] C. M. Gibson, C. P. Cannon, W. L. Daley, J. T. Dodge, B. Alexander, S. J. Marble, C. H. McCabe, L. Raymond, T. Fortin, and W. K. Poole, "TIMI frame count a quantitative method of assessing coronary artery flow," Circulation 93(5), p. 879-888, 1996.

[2] J. H. C. Reiber, P. W. Serruys, and C. J. Slager, Quantitative coronary and left ventricular cineangiography : methodology and clinical applications, Developments in cardiovascular medicine, Boston ; Dordrecht : Nijhoff, 1986.

[3] M. Schrijver, Angiographic Image Analysis to Assess the Severity of Coronary Stenosis. PhD thesis, University of Twente, 2002.

[4] N. P. Csizmadia, M. Schrijver, C. H. Slump, and A. P. G. Lubbers, "Digital densitometric determination of relative coronary flow distributions," Medical and Biological engineering and computing 39, p. 303-309, Feb. 2001.

[5] M. G. Stoel, F. Zijlstra, and C. A. Visser, "Frame count reserve," Circulation 107, p. 3034-3039, June 2003.

[6] P. Fallavollita and F. Cheriet, "Optimal 3D reconstruction of coronary arteries for 3D clinical assessment.," Computerized Medical Imaging and Graphics 32(6), pp. 476-487, 2008.

[7] S. Y. J. Chen and C. E. Metz, "Improved determination of biplane imaging geometry from two projection images and its application to threedimensional reconstruction of coronary arterial trees," Medical Physics 24, p. 633, 1997. 
[8] S. Y. J. Chen and J. D. Carroll, "Kinematic and deformation analysis of 4-D coronary arterial trees reconstructed from cine angiograms," Medical Imaging, IEEE Transactions on 22(6), p. 710-721, 2003.

[9] C. Blondel, G. Malandain, R. Vaillant, and N. Ayache, "Reconstruction of coronary arteries from a single rotational x-ray projection sequence," IEEE Transactions on Medical Imaging 25(5), p. 653-663, 2006.

[10] R. Gordon, R. Bender, and G. T. Herman, "Algebraic reconstruction techniques (ART) for three-dimensional electron microscopy and x-ray photography," Journal of Theoretical Biology 29, p. 471-481, Dec. 1970.

[11] M. Li, H. Yang, and H. Kudo, "An accurate iterative reconstruction algorithm for sparse objects: application to 3D blood vessel reconstruction from a limited number of projections," Physics in Medicine and Biology 47(15), p. 2599-2609, 2002.

[12] M. Li, H. Kudo, J. Hu, and R. H. Johnson, "Improved iterative algorithm for sparse object reconstruction and its performance evaluation with microCT data," Nuclear Science, IEEE Transactions on 51(3), p. 659-666, 2004 .

[13] E. Hansis, D. Schäfer, O. Dössel, and M. Grass, "Evaluation of iterative sparse object reconstruction from few projections for 3 -D rotational coronary angiography.," IEEE transactions on medical imaging 27(11), p. $1548,2008$.

[14] O. Wink, R. Kemkers, S. Y. Chen, and J. D. Carroll, "Intra-procedural coronary intervention planning using hybrid 3-dimensional reconstruction techniques," Academic radiology 10(12), p. 1433-1441, 2003.

[15] K. R. Hoffmann, A. Wahle, C. Pellot-Barakat, J. Sklansky, and M. Sonka, "Biplane x-ray angiograms, intravascular ultrasound, and 3D visualization of coronary vessels," The International Journal of Cardiac Imaging 15, p. 495-512, Dec. 1999.

[16] G. A. ten Brinke, C. H. Slump, and C. J. Storm, "Digital densitometric determination of clinical relative coronary flow distributions," in Proceedings of the SPIE, 6143, International Society for Optical Engineering, 2006 .

[17] R. Hartley and A. Zisserman, Multiple View Geometry in Computer Vision, Cambridge University Press, 2000.

[18] A. Amini, T. E. Weymouth, and R. C. Jain, "Using dynamic programming for solving variational problems in vision," IEEE transactions on pattern analysis and machine intelligence 12(9), p. 855-867, 1990. 
[19] E. W. Dijkstra, "A note on two problems in connexion with graphs," Numerische Mathematik 1, pp. 269-271, Dec. 1959.

[20] J. A. Sethian, "Evolution, implementation, and application of level set and fast marching methods for advancing fronts," Journal of Computational Physics 169(2), p. 503-555, 2001.

[21] M. S. Hassouna and A. A. Farag, "Multistencils fast marching methods: A highly accurate solution to the eikonal equation on cartesian domains," IEEE Transactions on Pattern Analysis and Machine Intelligence 29(9), p. 1563-1574, 2007.

[22] J. Canny, "A computational approach to edge detection," IEEE Transactions on Pattern Analysis and Machine Intelligence 8, p. 679-698, 1986.

[23] J. Illingworth and J. Kittler, "A survey of the hough transform," Computer Vision, Graphics, and Image Processing 44(1), p. 87-116, 1988. 


\section{CHAPTER 9}

\section{Conclusion and Recommendations}

\subsection{Conclusion}

Quantitative analysis of coronary blood flow velocity can be a predictive measure for coronary stenosis. In this thesis the subject of finding coronary blood flow velocity using the standard clinical procedure for coronary intervention is assessed. In this chapter we will answer the research question which are posted in the introduction, section 1.4 .

\section{Clinical coronary assessment methods}

In chapter 2 we discussed x-ray angiography. The following questions can be answered from this chapter:

- How can we quantify coronary stenosis?

Visually following the bolus after injection in the coronary arteries leads to a propagation time through the coronary arteries. This time is longer in the occurrence of coronary lesions, like stenosis, see figure 2.7.

- What is the clinical equivalent to measure coronary blood flow velocity from x-ray images?

The TIMI study group has proposed TIMI frame counting to assess this propagation time. It appears that, at a frame rate of $30 \mathrm{fps}$, coronary flow velocity can be quantized. The accuracy is related to the frame rate, which is the temporal resolution. If the frame rate is lowered, the accuracy is decreasing. Stoel 
et al. (2003) proposed to use the difference in TFC in hyperemic and basal state to calculate a rational called frame count reserve. It appears that this ratio is related to coronary flow reserve. It can be concluded that the angiographic data contain information about coronary flow reserve. From this point of view we propose to automate the clinical TIMI frame counting method.

\section{Finding time density curves}

Flow velocity can be estimated when the arrival times of the contrast agent along the coronary artery can be detected. In chapter 3 we proposed to track the arteries and place regions of interest equispaced along the vessel. With this measurement technique, multiple time density curves are measured, from which a flow velocity estimation is calculated.

- How can we automatically find the vascular structure in 2-d x-ray images?

We have used tube filtering algorithms to find the vascular structure. A severe limitation of these algorithms is the inability to correctly detect bifurcations, leaving holes at the place of a bifurcation. Manual annotation is used because of its reliabilty. However, we have used vessel detection algorithms to support the manual annotation.

- How can we accurately extract time density curves?

The key challenge with the extraction of time density curves is the ability to track the arteries. Artery tracking is a difficult task, since there are not many fiducial points in the images. Moreover, we are dealing with the projection of a 3-d structure which poses non-linear behavior when tracking a point on an artery. We proposed to solve this problem using a 3 -d model in chapter 7 .

- Are time density curves sufficient to determine coronary flow?

We found that the number of regions where the contrast density is measured is sufficient to determine coronary flow. However, the quality of the time density curves strongly depends on the quality of the tracking of the vessel. We think that more accurate flow estimations can be obtained by using the contrast density along the vessel centerline.

\section{Flow map introduction}

In chapter 4 we introduced the concept of a flow map, which is a 2 -d representation of a set of time density curves with a higher spatial resolution. We explored the usage of this flow map to answer the following research questions:

- How do we represent a set of time density curves? 
Figure 4.1 shows the representation of the flow map. The flow map consists of the contrast density along the vessel centerline on the first axis and the time on the second axis. The vessel centerline is found using the fast marching method. This assures that we find the lowest cost path, which represents the shortest path with the highest contrast density.

- Can we estimate flow velocity from a flow map?

The 2-d data of the flow map can be analyzed using standard image processing methods. In this case we assume that the flow velocity is a linear process, thus fitting a line and finding the slope using the Hough transform gives us an estimate of the flow velocity.

\section{3-d reconstruction of the coronary arteries}

In chapter 5 we discussed the mathematics behind bi-plane reconstruction and the application to coronary x-ray images. Main question answered in this chapter is:

- Contain x-ray images enough information to reconstruct 3-d models?

Reconstruction of coronary arteries in the three dimensional domain using only two images is a challenge, especially when the geometry of the acquisition is not completely defined. We have implemented a 3 -d modeling algorithm and have used bifurcation, begin and end point annotation to define fiducial points, which we used in the epipolar matching process.

\section{4-d modeling of coronary arteries}

The next step was the creation of a time varying model of the coronary arteries and measure the contrast density using the 4-d model. The key challenge was the three dimensional reconstruction of the coronary vessel tree and subsequently the temporal modeling of the coronary vasculature. Assuming that the temporal reconstruction results in a valid coronary model covering the whole cardiac cycle, a valid flow map was created. This was done by fitting the 4$\mathrm{d}$ model to the 2-d image sequence with contrast enhanced coronary arteries. Measurement of the vessel centerline resulted in the flow map, which was used to measure coronary flow velocity.

- How can we create flow maps using a 4-d model of the coronary arteries during the whole cardiac sequence?

In chapter 6 we presented a 3-d model using b-splines. At each phase of the cardiac cycle, these b-splines were fitted to images belonging to this cardiac phase using retrospective gating. This left us with a $4-\mathrm{d}(3-\mathrm{d}+\mathrm{t})$ model of the coronary arteries covering the complete cardiac cycle. This 4-d model was used to locate the vessels in the x-ray image sequence from which a flow map was generated. 


\section{Validation of our method using clinical data}

The final chapter of this thesis compared clinical and computer analyzed flow velocity estimations. The remaining questions are:

- How accurate is our algorithm?

In chapter 8 we compared the clinical measurements of the flow velocity to the automated measurements. Although we worked with uncalibrated data and an image set which was not acquired for the purpose of being reconstructed in 3-d, we managed to get a correlation of the measured TFC and the clinical measured TFC of $\mathrm{r}=0.98(\mathrm{P}<0.0001,95 \%$ CI 0.92 to 0.99$)$ on 9 patients of our dataset.

- What are the limitations of our algorithm?

The proposed method has limitations: First of all, the frame rate is a limiting factor in the temporal resolution of the flow measurements. Secondly, the contrast ratio of the opacified arteries and the background is not always maximized, making it difficult to find vessels and discriminate vessels from other structures like bones. Furthermore, coronary arteries are not always opacified completely during one cardiac cycle, this is required when creating a 3 -d reconstruction of the cardiac cycle. Also, a minimum of two different projection angles is required for the 3 -d reconstruction.

\subsection{Recommendations}

A software package to get TDCs from the angiograms is now available. Artery tracing fails when the opacification of the vessel tree is not complete, resulting in branches that end at places where they normally would continue. Therefore, this algorithm should be expanded with the knowledge that vessels with a certain diameter are expected to continue. This improvement can be achieved by a model fitting approach. Also, all manual correction steps should be automated. If the tracing and tracking algorithm can be perfected to run completely autonomous without errors, than higher quality TDCs can be extracted and a more detailed analysis of these curves can be performed. 


\section{List of publications}

[1] G. A. ten Brinke, C. H. Slump, and C. J. Storm, "Digital densitometric determination of clinical relative coronary flow distributions," in Proceedings of the SPIE, 6143, International Society for Optical Engineering, 2006.

[2] G. A. ten Brinke, R. G. M. Kolkman, C. H. Slump, and W. Steenbergen, "Photoacoustic 3D visualization of tumor angiogenesis," in Proceedings of SPIE, 6920, International Society for Optical Engineering, 2008.

[3] R. G. M. Kolkman, K. K. Thumma, G. A. ten Brinke, R. I. Siphanto, H. van Neck, W. Steenbergen, and T. G. van Leeuwen, "Photoacoustic imaging of tumor angiogenesis," in Proceedings of SPIE, pp. 685602-685602-6, International Society for Optical Engineering, 2008.

[4] G. A. ten Brinke, C. H. Slump, M. G. Stoel, and C. J. Storm, "A method towards automated thrombolysis in myocardial infarction (TIMI) frame counting using 3D reconstruction," 2009, p. 653-656, Computers in Cardiology, 2009.

[5] G. A. ten Brinke, C. H. Slump, and C. J. Storm, "Bi-Plane x-ray coronary 3D reconstruction for flow velocity assessment.," in Proceedings of the IEEE-EMBS Benelux Chapter 2009, IEEE EMBS Benelux Chapter, (Enschede), 2009.

[6] G. A. ten Brinke, M. G. Stoel, and C. H. Slump, "Automatic TIMI frame counting using 3-d modeling," submitted . 


\section{APPENDIX A}

\section{Datasets}

Two datasets, $A$ and $B$, have been used in this research. Dataset $A$ contains specific patient data for the analysis of basal and hyperemic flow using TIMI frame counting.

\section{A.1 Dataset A}

\section{A.1.1 Patients}

In 38 selected patients referred for diagnostic coronary angiography or PCI for stable angina, FCR and CVR were calculated for both left anterior descending (LAD) and left circumflex coronary artery (LCx). At least 1 of these 2 vessels was normal (visually no stenosis $>50 \%$ ), and all vessels had TIMI 3 flow. In the case of PCI, measurements were repeated thereafter. No patient had left main stenosis $>30 \%$, history of obstructive pulmonary disease, or myocardial infarction in the territory of the left coronary artery, and all patients had sinus rhythm. The Medical Ethics Committee of the hospital of the Free University Amsterdam, the Netherlands, approved the protocol. All patients gave written informed consent.

\section{A.1.2 Procedure}

One operator (M.S.), using 7F guiding catheters without side holes (Guidant) and ionic contrast agent (Hexabrix, Guerbet), performed all procedures. At the beginning of the procedure, an intravenous bolus of heparin (5000 IU) was 
given. In all but 1 case of PCI, a stent was implanted; there were no residual stenoses $>30 \%$. More than 5 minutes before angiography (Philips Integris 3000, 25 frames/s) and Doppler measurements (Cardiometrics), 0.2 to $0.4 \mathrm{mg}$ intracoronary nitroglycerine was given. Angiography (right anterior oblique with caudal angulation without magnification) was performed by manual injection through the contrast-filled guiding catheter, synchronized to an acoustic heart beat signal. It was performed at least 10 minutes after last balloon inflation and 2 minutes after last contrast injection to prevent reactive hyperemia attributable to ischemia or contrast agent.

\section{A.1.3 Protocol and Calculations}

After positioning the Doppler wire in a normal segment of LAD or LCx, as proximal as possible but $>2 \mathrm{~cm}$ distal to the culprit lesion if present, basal average peak velocity (APV) was recorded together with blood pressure and heart rate. Angiography was performed for basal TFC of both vessels. Hyperemia was induced by adenosine, $140 \mu \mathrm{g} / \mathrm{kg}$ per min intravenous for at least 3 minutes. Hyperemic APV was recorded, and contrast injection for hyperemic TFC was given. The Doppler wire was then repositioned in the ipsilateral vessel with recording of hyperemic APV, after which adenosine was stopped. More than 3 minutes later, basal APV of this vessel was recorded. CVR was determined by dividing hyperemic by basal APV. With the digitally numbered frames, TFC was calculated as described before.15 FCR was calculated by dividing basal by hyperemic TFC. Relative CVR and rFCR were determined by the ratio of the reserve of $\mathrm{LCx}$ and $\mathrm{LAD}$. The frames between the last QRS complex and the first frame of the TFC were counted. By dividing this number by 25 (frames/s), an estimate was made of the beginning of the contrast injection in relation to the phase of the cardiac cycle. Length of both coronary arteries was measured by using the Doppler or regular guidewire, as described by Gibson et al. [1]. The distal end of the guidewire was placed 1 to $3 \mathrm{~mm}$ in the distal landmark, marked outside the proximal end of the guiding catheter, then pulled back until 1 to $3 \mathrm{~mm}$ was still visible outside the distal end of the guiding catheter and marked again. The measured distance between the 2 marks was used to estimate the length of the coronary artery from the tip of the guiding catheter to the landmark used for the TFC. By multiplying this length by $25 / \mathrm{TFC}$, FCV was calculated. The diameter of the proximal segment of the coronary artery and the percentage stenosis of the target lesion if present was calculated by quantitative coronary angiography as the mean of 2 orthogonal measurements using the contrast-filled catheter as reference diameter. Coronary volume flow was estimated by the following equation: flow $(\mathrm{mL} / \mathrm{min})=\mathrm{FCV}(\mathrm{cm} / \mathrm{s}) \times 60 \times \pi \times(\text { diameter } / 2)^{2}$. A coronary vascular resistance index $(\mathrm{mm} \mathrm{Hg}$ per $\mathrm{mL} / \mathrm{min})$ was calculated by dividing mean arterial blood pressure by coronary flow, assuming right atrium pressure was zero. 


\section{A.2 Dataset B}

This dataset contains images of 34 patients imaged using a Philips Integris $\mathrm{H}$ and 23 patients imaged with a Siemens Axiom-Artis. The aquisition is performed using standard protocols.

\section{References}

[1] M. D. Gibson, C. Michael, J. T. D. Jr, M. D. Goel, et al., "Angioplasty guidewire velocity: a new simple method to calculate absolute coronary blood velocity and flow," The American journal of cardiology 80(12), pp. $1536-1539,1997$. 
Dit is het moment waarop ik de kans heb om iedereen te bedanken die direct of indirect heeft meegeholpen bij het tot stand komen van dit proefschrift. Ik zal mij hier bewust beperken tot de 4 jaar promotie op de universiteit, uiteraard zijn er nog veel meer mensen geweest die ik hier niet allemaal persoonlijk kan bedanken.

In de eerste plaats wil ik mijn promotor Kees Slump bedanken voor het beschikbaar stellen van een promotieplaats waarin ik complete vrijheid heb gekregen en mij hierdoor forceerde om mijn eigen onderzoek en richting te kiezen. Ik heb ontzag gekregen voor de manier waarop je kritisch kunt zijn zonder direct kritiek te leveren.

Op de tweede plaats wil ik de cardiologen Cock Storm en Martin Stoel bedanken voor hun bijdrage in de vorm van patientendata. Vooral het onderzoek van Martin heeft erg veel bijgedragen omdat zijn patientendata perfect aansloot bij het onderwerp van dit proefschrift.

Bijzonder interessant vond ik de multidisciplinaire bijeenkomsten in het kader van het NIMTIK programma waaronder dit onderzoek ook viel, ondanks dat sommige onderwerpen wel erg ver van image processing af lagen.

Verder wil ik mijn collega's bedanken die de ELTN/Hogekamp en Carre tot een fantastische werkplek hebben gemaakt. Het was altijd gezellig met de secretaresses Anneke en Sandra en met mijn altijd vrolijke kamergenoot Xiaoying. Niet te vergeten de vrijdag rond 4 uur waarin Geert-Jan borrel schreeuwt en een select gezelschap nog de stad in gaat en via de Griek in de Paddy's beland. Waaronder Roel, Gert, Anne, Bas en Rene die het wel tot 4 uur 's nachts konden volhouden. Dan hebben we nog de conferenties met bijbehorende tripjes naar o.a. de NASA met Rene, Dirk-Jan en Almar. Ik zal Las Vegas en Miami beach niet snel vergeten, wie gelooft er nu dat een fotomodel PIC processortjes programmeren leuk vind? Ook dit jaar gaan we 
weer met een gedeelte van SAS op wintersport, Val Thorens is de bestemming en dit is niet geheel toevallig tijdens de vakantie van de Belgen.

Natuurlijk kan ik ook de collega AIO's van Jelle's leerstoel niet vergeten: Tom, Sebastian, Mariet, Maaike, Paul, Ivo en Quint bedankt voor de vele gezellige FCRE borrels en etentjes.

Mijn studie op de universiteit heb ik gedeeld met een speciale HBO instroom groep, vanuit deze groep zijn we met vijf man gaan promoveren. Marcus, Arno, Alex en Mark jullie zijn allemaal inmiddels gepromoveerd, ik kan dan natuurlijk niet achterblijven. Stappen in Amsterdam, Leuven of in de Pallaz in Eindhoven?

Tijdens mijn promotie ben ik lid geworden van zweefvliegclub Vleugellam. Hier heb ik, behalve leren vliegen, ook twee jaar lang het penningmeesterschap op mij genomen. In het bijzonder wil ik hier Olivier, Evert, Gert-Jan, Jan-Willem, Wim en Joeri bedanken voor het delen van alle tegenslagen en overwinningen.

Het weekend was natuurlijk de perfecte uitlaatklep, menig glaasje met bubbels is gedeeld met Bob, Christiaan, Ernst-Jan, Firdanus, Jonny en Tom.

Als laatste wil ik mijn familie waaronder Pa, Ma, Genanja en Edwin, Anno en Anne en natuurlijk Jelle bedanken. Jelle, de afgelopen negen jaar met jou waren fantastisch en ik hoop dat er nog veel zullen volgen.

Gerbert

Eindhoven, November 2011 